\title{
HOMOLOGICAL ALGEBRA IN LOCALLY COMPACT ABELIAN GROUPS
}

\author{
BY \\ MARTIN MOSKOWITZ $\left({ }^{1}\right)$
}

I. Introduction. This paper is concerned with homological algebra in the category $\mathscr{L}$ of locally compact abelian topological groups. The morphisms of $\mathscr{L}$ are the continuous homomorphisms. However, only certain exact sequences, resolutions etc., are admissible. These are sequences whose continuous homomorphisms are open onto their respective ranges. Such maps are called proper and the corresponding sequences, proper exact. Concomitant with this is the fact that although $\mathscr{L}$ is an additive category it is not abelian.

The material in §II may be regarded as preparatory, although there may be some independent interest here. Various structural facts are proven (some of which are well known) and basic properties of important dual subcategories of $\mathscr{L}$ are investigated.

In $\S I I I$ the projectives and injectives of $\mathscr{L}$ are computed. It turns out that subgroups of projectives are projective and quotient groups of injectives are injective. Vector groups are characterized by the fact that they are both projective and injective. Finally, necessary and sufficient conditions are given for the existence of proper resolutions.

In $\$ I V$ and $V$ continuous versions of the functors Hom and $\otimes$ (via dualization) are defined on certain subcategories of $\mathscr{L}$ and their functorial properties, including exactness, are investigated. These extend the usual notions for discrete groups. In order to do this it is necessary to study topological groups of continuous multilinear functions. Sufficient, and in a sense, necessary conditions are given for the various groups to be locally compact. Under these conditions the appropriate functors are shown to be isomorphic. Finally, more or less explicit computations are made for Hom, $\otimes$, etc., sharpening some of the earlier results, and their geometric and structural significance is investigated.

In $\S$ VI Tor and Ext are defined by resolutions as derived functors of $\otimes$ and Hom, and their functorial properties are studied. It turns out that Tor ${ }_{n}$ and $\mathrm{Ext}_{n}$ vanish for $n \geqq 2$, and that Tor ${ }_{1}$ and Ext ${ }_{1}$ are computable. Knowledge of Ext $_{1}$ in turn gives information about certain group extensions in $\mathscr{L}$.

$\mathrm{Th}$, hout this paper, complete duality of all concepts and theorems is obtained. In order to accomplish this some concessions have to be made to the topology at various stages. For this reason the functors Hom, $\bigotimes$, Tor, and Ext are not defined on $\mathscr{L}$ but only on certain subcategories.

Received by the editors September 7, 1965.

(1) Research partially supported by the National Science Foundation. 
This paper relies heavily on the Pontrjagin Duality Theorem and related structural and character theoretic facts about locally compact abelian groups. These facts are to be found for the most part in [1] or [2]. Material on discrete abelian group theory, and homological algebra are to be found in [3] and [6] respectively. Whenever possible we have deliberately chosen to refer to textbooks rather than the original papers since there the material is systematically organized. One theorem of some importance in the theory of locally compact groups is the so-called Open Mapping Theorem due to Pontrjagin. (See [2, Vol. I].) Since it seems to be less well known than the above material and is used so frequently here, it is worthwhile stating.

Let $G$ and $H$ be arbitrary locally compact groups and $f: G \rightarrow H$ a continuous surjective homomorphism. If $G$ is the countable union of compact sets, then $f$ is open.

Now a locally compact group $G$ is called compactly generated if there exists a compact symmetric neighborhood $U$ of 1 so that $G=\bigcup_{n=1}^{\infty} U^{n}$. Evidently a compactly generated group is the countable union of compact sets, and it is the class of compactly generated abelian groups to which we will apply the Open Mapping Theorem.

Before proceeding we make the following notational conventions: By $G$ in $\mathscr{L}$ we mean that $G$ is a locally compact abelian group with the group operation written additively. We denote by $R, Z, T$ the group of real numbers, integers and reals modulo 1 respectively. If $G$ and $H$ are in $\mathscr{L}$ and $f: G \rightarrow H$ is a continuous homomorphism we denote by $G^{\wedge}$ and $H^{\wedge}$ the character groups of $G$ and $H$ and by $f^{\wedge}: H^{\wedge} \rightarrow G^{\wedge}$ the continuous homomorphism dual to $f$. If $G$ is in $\mathscr{L}$ and $H$ is a closed subgroup of $G$, then $\left(G^{\wedge}, H\right)$ stands for the annihilator of $H$ in $G^{\wedge}$. The symbol $\cong$ always means an isomorphism of topological groups. If $\left\{G_{i}\right\}_{i \in I}$ is a family of groups in $\mathscr{L}$ we denote their direct product by $G_{1} \oplus \cdots \oplus G_{n}$ if the family is finite and by $\prod_{i \in I} G_{i}$ in general. If all the $G_{i}$ are equal we shall write $G^{I}$. The group $G$ always denotes a locally compact abelian group, and $G_{0}$ its identity component. The symbols $S^{\circ}, S^{-}$, and id ${ }_{S}$ denote the interior, closure, and identity map $S \rightarrow S$ respectively.

Finally, I would like to express my gratitude and appreciation to Professor G. P. Hochschild who, as thesis adviser, gave without limit his time, energy, and advice. In addition to many valuable suggestions Professor Hochschild gave me much encouragement during the entire period of the preparation of this paper.

\section{Structural results.}

Proposition 2.1. Let $G_{1}$ and $G_{2}$ be in $\mathscr{L}, f: G_{1} \rightarrow G_{2}$ be a continuous homomorphism, and $G_{1}^{\wedge}, G_{2}^{\wedge}$ and $f^{\wedge}$ be their duals. Then,

(1) $\operatorname{Ker} f^{\wedge}=\left(G_{2}^{\wedge}, f\left(G_{1}\right)^{-}\right)$,

(2) $f^{\wedge}\left(G_{2}\right)^{-}=\left(G_{1}^{\wedge}, \operatorname{Ker} f\right)$,

(3) $f^{\wedge}$ is a monomorphism if and only if $f\left(G_{1}\right)$ is dense in $G_{2}$.

Proof. (1) It is easy to see that for any closed subgroup $H_{1}$ of $G_{1}$,

$$
\left(f^{\wedge}\right)^{-1}\left(G_{1}^{\wedge}, H_{1}\right)=\left(G_{2}^{\wedge}, f\left(H_{1}\right)^{-}\right) \text {. }
$$

The result follows by taking $H_{1}=G_{1}$. 
(2) Apply (1) to $f^{\wedge}$. Then $\operatorname{Ker} f^{\wedge \wedge}=\left(G_{1}^{\wedge}, f^{\wedge}\left(G_{2}^{\wedge}\right)^{-}\right)$and therefore, by the duality theorem, $\operatorname{Ker} f=\left(G_{1}, f^{\wedge}\left(G_{2}\right)^{-}\right)$. Taking annihilators we have

$$
f^{\wedge}\left(G_{2}^{\wedge}\right)^{-}=\left(G_{1}^{\wedge}, \operatorname{Ker} f\right) .
$$

(3) $f^{\wedge}$ is a monomorphism if and only if $(0)=\operatorname{Ker} f^{\wedge}=\left(G_{2}^{\wedge}, f\left(G_{1}\right)^{-}\right)$, i.e., if and only if $f\left(G_{1}\right)^{-}=G_{2}$.

Proposition 2.2. Let $G_{1}$ and $G_{2}$ be in $\mathscr{L}$. If : $G_{1} \rightarrow G_{2}$ is a proper homomorphism then

(1) $f\left(G_{1}\right)$ is a closed subgroup of $G_{2}$,

(2) $\operatorname{Ker} f^{\wedge}=\left(G_{2}^{\wedge}, f\left(G_{1}\right)\right)$,

(3) $f^{\wedge}$ is a proper homomorphism.

Proof. (1) Since $f\left(G_{1}\right)$ is a continuous open image of a locally compact space, it is locally compact. A locally compact subgroup of a topological group is closed.

(2) This follows immediately from (1) and Proposition 2.1, Part 1.

(3) $f$ factors into $g \pi$ where $\pi: G_{1} \rightarrow G_{1} / \operatorname{Ker} f$ is the canonical epimorphism and $g: G_{1} / \operatorname{Ker} f \rightarrow G_{2}$ is the continuous monomorphism induced by $f$. Since $f=g \pi$ where $\pi$ is continuous, and $f$ is a proper homomorphism, $g$ is a proper monomorphism.

Now, as is known (see [2]), the dual $\pi^{\wedge}$ of the proper epimorphism $\pi$ is a proper monomorphism; and the dual $g^{\wedge}$ of the proper monomorphism $g$ is a proper epimorphism. So $f^{\wedge}=\pi^{\wedge} g^{\wedge}$ and is therefore an open map followed by an open map onto its range.

Definition. A sequence

$$
\text { (S) } \cdots \longrightarrow G_{i} \stackrel{f_{i}}{\longrightarrow} G_{i+1} \stackrel{f_{i+1}}{\longrightarrow} G_{i+2} \longrightarrow \cdots
$$

where each $G_{i}$ is in $\mathscr{L}$ and each $f_{i}$ is a continuous homomorphism is called a complex if $f_{i}\left(G_{i}\right) \subset \operatorname{Ker} f_{i+1}$ for all $i$. The sequence is called exact if for each $i, f_{i}\left(G_{i}\right)=\operatorname{Ker} f_{i+1}$. A complex or an exact sequence is called proper if each $f_{i}$ is a proper homomorphism. Note that if the $G_{i}$ are compactly generated then an exact sequence is automatically proper exact by the Open Mapping Theorem.

THEOREM 2.1. If the sequence

$$
\text { (S) } \cdots \longrightarrow G_{i} \stackrel{f_{i}}{\longrightarrow} G_{i+1} \stackrel{f_{i+1}}{\longrightarrow} G_{i+2} \longrightarrow \cdots
$$

is proper exact then its dual

$$
\cdots \longrightarrow G_{i+2} \stackrel{f_{i+1}}{\longrightarrow} G_{i+1} \stackrel{f_{i}^{\wedge}}{\longrightarrow} G_{i} \longrightarrow \cdots
$$

is also proper exact. In particular, the dual of a short proper exact sequence $0 \rightarrow G_{1} \rightarrow G_{2} \rightarrow G_{3} \rightarrow 0$ is short proper exact. 
Proof. By Proposition 2.2, $f_{i}^{\wedge}$ is a proper homomorphism and

$$
\operatorname{Ker} f_{i}^{\wedge}=\left(G_{i+1}^{\wedge}, f_{i}\left(G_{i}\right)\right) \text {. }
$$

But $\left(G_{i+1}, f_{i}\left(G_{i}\right)\right)=\left(G_{i+1}^{\wedge}, \operatorname{Ker} f_{i+1}\right)$ since $f_{i}\left(G_{i}\right)=\operatorname{Ker} f_{i+1}$. Proposition 2.2 implies that $\left(G_{i+1}, \operatorname{Ker} f_{i+1}\right)=f_{i+1}\left(G_{i+2}\right)$. Thus $\operatorname{Ker} f_{i}^{\wedge}=f_{i+1}\left(G_{i+2}\right)$.

REMARK. It is clear that the dual of a complex is a complex and that of a proper complex is a proper complex.

Proposition 2.3. Let $G$ be in $\mathscr{L}$ and $H_{1}$ and $H_{2}$ closed subgroups of $G$ with $H_{1} \subset H_{2}$. Then $\left(H_{2}^{\wedge}, H_{1}\right) \cong\left(G^{\wedge}, H_{1}\right) /\left(G^{\wedge}, H_{2}\right)$.

Proof. Consider the short proper exact sequence

$$
0 \rightarrow H_{2} / H_{1} \rightarrow G / H_{1} \rightarrow G / H_{2} \rightarrow 0 .
$$

As is known (see [2]), the dual sequence is

$$
0 \rightarrow\left(G^{\wedge}, H_{2}\right) \rightarrow\left(G^{\wedge}, H_{1}\right) \rightarrow\left(H_{2}^{\wedge}, H_{1}\right) \rightarrow 0
$$

and is proper exact by Theorem 2.1 .

THEOREM 2.2. Let the sequence (S) be a proper complex and

$$
\cdots \longleftarrow G_{i}^{\wedge} \stackrel{f_{i}^{\wedge}}{\longleftarrow} G_{i+1} \stackrel{f_{\hat{i+1}}}{\longleftarrow} G_{i+2} \longleftarrow \cdots
$$

be its dual. Denote by $H^{i}$ the ith cohomology group of the former sequence and $H_{i}$ the ith homology group of the latter. Then $H^{i}$ and $H_{i}$ are in $\mathscr{L}$ and $H^{i}=H_{i}$.

Proof. The fact that both complexes are proper implies that $H^{i}$ and $H_{i}$ are in $\mathscr{L}$ by Proposition 2.2. The proof will be complete if we can show that for each $i$, $\left(H^{i+1}\right)^{\wedge}=H_{i+1}$. By Proposition 2.2, Ker $f_{i}^{\wedge}=\left(G_{i+1}, f_{i}\left(G_{i}\right)\right)$ and $f_{i+1}\left(G_{i+2}\right)$ $=\left(G_{i+1}^{\hat{n}}, \operatorname{Ker} f_{i+1}\right)$. Hence $\operatorname{Ker} f_{i} \mid f_{i+1}\left(G_{i+2}\right)=\left(G_{i+1}, f_{i}\left(G_{i}\right)\right) /\left(G_{i+1}, \operatorname{Ker} f_{i+1}\right)$. However, the latter term is isomorphic with $\operatorname{Ker}\left(f_{i+1}, f_{i}\left(G_{i}\right)\right)$ which as is known (see [2]) is isomorphic with $\left(\operatorname{Ker} f_{i+1} / f_{i}\left(G_{i}\right)\right)$.

Next we investigate the structure of the class of groups without small subgroups.

Definition. A group $G$ in $\mathscr{L}$ has no small subgroups if there exists a neighborhood $U$ of 0 which does not contain any nontrivial subgroups.

Proposition 2.4. A locally compact group without small subgroups has a countable fundamental system of neighborhoods of $(0)$.

Proof. Let $U_{0}$ be a compact symmetric neighborhood of (0) containing no nontrivial subgroups of $G$. Construct a sequence $U_{0}, U_{1}, \ldots$ of compact symmetric neighborhoods of $(0)$ such that $2 U_{i} \subset U_{i-1}$ for all $i>0$. Then $\bigcap_{i=0}^{\infty} U_{i}$ is evidently a subgroup of $G$ contained in $U_{0}$. Hence $\bigcap_{i=0}^{\infty} U_{i}=(0)$. Now let $U$ be any neighborhood of (0) in $G$. $U$ contains an open neighborhood $U^{\prime}$ of $(0)$ such that $U^{\prime} \subset U_{0}$. Consider the family of closed subsets $U_{i} \cap \tilde{U}^{\prime}$ of the compact space $U_{0} \cap \tilde{U}^{\prime}$ where $\tilde{U}^{\prime}$ is the complement of $U^{\prime}$ in $G$. The intersection of any finite subfamily 
of these is again a $U_{j} \cap \tilde{U}^{\prime}$, and the intersection of all of these is empty. Hence there exists an $i$ so that $U_{i} \cap \tilde{U}^{\prime}=\varnothing$, so that $U_{i} \subset U^{\prime} \subset U$. Therefore, the $U_{i}$ 's are a countable fundamental system of neighborhoods of $(0)\left({ }^{2}\right)$.

Definition. A test neighborhood $\left({ }^{3}\right)$ of a group $G$ in $\mathscr{L}$ is a neighborhood $\Gamma_{*}$ of (0) in $G$ such that for each neighborhood $\Gamma$ of $(0)$ in $G$ there is an integer $k(\Gamma)$ which has the property that for every $x$ in $G$, if $x, 2 x, \ldots, k(\Gamma) x \in \Gamma_{*}$, then $x \in \Gamma$.

THEOREM 2.3. A group $G$ has a test neighborhood if and only if it has no small subgroups.

Proof. Suppose $G$ is a locally compact abelian group without small subgroups. Let $\Gamma_{*}$ be a compact symmetric neighborhood of $(0)$ which contains no subgroups, and $\Gamma$ be any neighborhood of $(0)$ in $G$. By looking at $\Gamma \cap \Gamma_{*}$ we may assume that $\Gamma \subset \Gamma_{*}$. If there is no integer $k(\Gamma)$ with the above property, then for each integer $k$ there is an $x(k)$ in $G$ such that $x(k), 2 x(k), \ldots, k x(k) \in \Gamma_{*}$, but $x(k) \notin \Gamma . \Gamma_{*}$ is compact, and by Proposition $2.4, G$ obeys the first axiom of countability. Hence there exists a subsequence $x\left(k_{i}\right) \rightarrow_{i} y$, where $y \in \Gamma_{*}$. Let $j$ be any fixed positive integer. Choose $i$ so that $k_{i}>j$. Then $j x\left(k_{i}\right) \in \Gamma_{*}$. But $j x\left(k_{i}\right) \rightarrow_{i} j y \in \Gamma_{*}$. Since $\Gamma_{*}$ is symmetric, it contains the subgroup generated by $y$. Thus $y=0$. Since $x\left(k_{i}\right) \rightarrow_{i} 0$, there exists an $i$ after which $x\left(k_{i}\right) \in \Gamma$. This contradicts the assumption.

Conversely, let $\Gamma_{*}$ be a test neighborhood of $(0)$. Suppose $\Gamma_{*}$ contains a subgroup $H$. Let $x \in H$. Then $x$ belongs to every neighborhood of (0), so that $x=0$. Thus $\Gamma_{*}$ contains no nontrivial subgroups.

Proposition 2.5. Let $G$ be in $\mathscr{L}$, and let $H$ be a closed subgroup of $G$.

(1) If $G$ has no small subgroups, then $H$ has no small subgroups.

(2) If $H$ is open and has no small subgroups, then $G$ has no small subgroups.

Proposition 2.6. Let $G=G_{1} \oplus G_{2} \oplus \cdots \oplus G_{n}$. $G$ has no small subgroups if and only if $G_{i}$ has no small subgroups for $i=1, \ldots, n$.

The proofs of Propositions 2.5 and 2.6 are clear.

THEOREM 2.4. Let $G$ be in $\mathscr{L}$. Then $G$ has no small subgroups if and only if $G \cong R^{n} \oplus T^{m} \oplus D$ where $R^{n}$ is an $n$-dimensional vector group, $T^{m}$ is an m-dimensional torus, and $D$ is a discrete group.

Proof. In any case, the general structure theorem for groups in $\mathscr{L}$ (see [1]) yields $G=R^{n} \oplus H$ where $H$ contains a compact open subgroup $C$. Now suppose that $G$ has no small subgroups. Then by Propositions 2.6 and 2.5, $C$ has no small subgroups. Because the characters separate the points of $C$, and $C$ is compact without small subgroups, $C$ is isomorphic to a closed subgroup of $T^{k}$, a finite-dimensional

(2) This proof is modeled after one of Yamabe's.

${ }^{(3)}$ The existence of a test neighborhood in $T$ was utilized by A. Weil in [1]. 
torus. This means that $C^{\wedge}$ is a quotient group of $Z^{k}$ and is therefore finitely generated. Hence $C^{\wedge} \cong Z^{m} \oplus F$ where $F$ is a finite group. Thus $C \cong T^{m} \oplus F$. Now $H / T^{m} / C / T^{m} \cong H / C$. But since $C / T^{m}$ is finite, and $H / C$ is discrete, it follows that $H / T^{m}$ is discrete, that is, $T^{m}$ is open in $H$. Since $T^{m}$ is divisible, it is a direct summand. So $H=T^{m} \oplus D$ where $D \cong H / T^{m}$, a discrete group. Therefore, $G \cong R^{n} \oplus T^{m} \oplus D$.

Conversely, since $R, T$, and $D$ clearly have no small subgroups, Proposition 2.6 implies that a group of the form $R^{n} \oplus T^{m} \oplus D$ has no small subgroups. This completes the proof. An immediate consequence is

COROLlaRY 1. If $G$ has no small subgroups then $G_{0}$, is open in $G$, and $G_{0} \cong R^{n} \oplus T^{m}$. Furthermore, $n, m$, and $D$ form a complete set of invariants for $G$.

COROLlARY 2. The following conditions are equivalent.

(1) $G$ is compact without small subgroups.

(2) $G \cong F \oplus T^{m}$ where $F$ is a finite group.

(3) $G^{\wedge}$ is discrete and finitely generated.

Proof. (1) and (2) are equivalent since if $G$ has no small subgroups and is compact, then $n=0$ and $D$ is finite, and conversely, (2) and (3) are equivalent by the Fundamental Theorem of Abelian Groups applied to $G^{\wedge}$.

Now we consider the class of compactly generated groups. The structure of these groups can be derived from Theorem 2.1 together with the general structure theorem and the fact that toral subgroups are direct summands (see 2). However, we make use of Theorem 2.4 which, in addition, yields the fact that they are dual to the class of groups without small subgroups.

THEOREM 2.5. Let $G$ be in $\mathscr{L}$. Then $G$ is compactly generated if and only if $G \cong R^{n} \oplus Z^{m} \oplus C$ where $n$ and $m$ are integers and $C$ is a compact group.

Proof. Suppose $G$ is compactly generated. Let $U$ be a compact neighborhood of (0), which generates $G$. Let $\Gamma_{*}$ be a neighborhood of $(0)$ in $T$ which contains no nontrivial subgroups. Denote by $W\left(U, \Gamma_{*}\right)$ the neighborhood of (0) in $G^{\wedge}$ made up of the characters $\xi$ of $G$ which take $U$ into $\Gamma_{*} . W\left(U, \Gamma_{*}\right)$ contains no nontrivial subgroups. Assume $n \xi \in W\left(U, \Gamma_{*}\right)$ for all integers $n$. Then $\xi(n U)$ $=(n \xi)(U) \subset \Gamma_{*}$ for all $n$, and so $\xi\left(\bigcup_{n=1}^{\infty} n U\right)=\xi(G) \subset \Gamma_{*} . \xi(G)$ is evidently a subgroup of $\Gamma_{*}$. Hence $\xi=0$. Thus, if $G$ is compactly generated then $G^{\wedge}$ has no small subgroups and is therefore isomorphic to $R^{n} \oplus T^{m} \oplus D$, by Theorem 2.4. Hence, $G \cong R^{n} \oplus Z^{m} \oplus G$.

Conversely, a group $G$ of the form $R^{n} \oplus Z^{m} \oplus C$ where $C$ is compact, is evidently compactly generated. It is clear that $n, m$, and $C$ are invariants and hence a complete set of invariants. $C$ is characterized by the fact that it contains every compact subgroup of $G$. $C$ is called the maximum compact subgroup of $G$.

COROLLARY 1. $G$ is compactly generated if and only if $G^{\wedge}$ has no small subgroups. This follows immediately from Theorems 2.4 and 2.5 . 
COROLlaRY 2. A compactly generated group $G$ has no nontrivial compact subgroups if and only if $G^{\wedge}$ is connected. $G$ is connected if and only if $G^{\wedge}$ is torsion free.

TheOREM 2.6. Let $G$ be in $\mathscr{L}$ and $H$ be a closed subgroup. Then

(1) $G$ has no small subgroups if and only if $H$ and $G / H$ have no small subgroups;

(2) $G$ is compactly generated if and only if $H$ and $G / H$ are compactly generated.

Proof. It suffices to prove (1) since (2) follows by dualization. Suppose that $H$ and $G / H$ have no small subgroups. Then there exists a neighborhood $U$ of (0) in $G$ such that $U \cap H$ contains no nontrivial subgroups, and also $\pi(U)$ contains no nontrivial subgroups, where $\pi$ is the canonical epimorphism $G \rightarrow G / H$. Now let $L$ be a subgroup of $G$, and suppose $L \subset U$. Then $\pi(L) \subset \pi(U)$, and therefore $L \subset H$. Since $L \subset U \cap H$, we know $L=(0)$.

Conversely, suppose $G$ has no small subgroups. By Proposition $2.5, H$ has no small subgroups. We show that $G / H$ has no small subgroups. First we observe that it is sufficient to show that $G_{0}+H / H$ has no small subgroups. In fact, as we noted in Theorem 2.4, Corollary $1, G_{0}$ and hence, $G_{0}+H$, is open in $G$. Now, $G / H / G_{0}+H / H \cong G / G_{0}+H$, which is discrete, and hence has no small subgroups. It then follows from the part of the theorem already proven that $G / H$ has no small subgroups.

Because $G_{0}$ is open, $G_{0}+H / H \cong G_{0} / G_{0} \cap H$. Thus it is sufficient to prove the theorem in the case that $G$ is connected, so that $G \cong R^{n} \oplus T^{m}$.

Consider the simply connected covering group $V$ of $G$ with $\phi$ the covering map. Let $\pi$ be the canonical map of $G$ onto $G / H \cdot \phi^{-1}(H)$ is a closed subgroup of $V$. Since $\phi$ and $\pi$ are continuous open epimorphisms, so is $\pi \phi$. Therefore, $G / H \cong V / \operatorname{Ker} \pi \phi$ which clearly has no small subgroups.

We give an application of our methods in proving the following known approximation theorem.

TheOREM 2.7. Let $G$ be in $\mathscr{L}$ and $U$ be a neighborhood of 0 in $G$. Then there exists a compact subgroup $H$ of $G$, so that $H \subset U$ and $G / H$ has no small subgroups; that is, any group in $\mathscr{L}$ is a projective limit of groups without small subgroups.

Proof. By taking $U$ sufficiently small and identifying $G$ with $G^{\wedge \wedge}$ we may assume $U=W(V, \Gamma)$ where $V$ is a compact symmetric neighborhood of 0 in $G^{\wedge}$, and $\Gamma$ is a neighborhood of 0 in $T$. Let $L=\bigcup_{n=1}^{\infty} n V$. Then

$$
0 \rightarrow L \rightarrow G^{\wedge} \rightarrow G^{\wedge} / L \rightarrow 0
$$

is a short proper exact sequence, where $L$ is compactly generated and $G^{\wedge} / L$ is discrete. By Theorem 2.1, the dual sequence is the short proper exact sequence $0 \rightarrow\left(G^{\wedge} / L\right)^{\wedge} \rightarrow G \rightarrow L^{\wedge} \rightarrow 0$. Let $H=\left(G^{\wedge} / L\right)^{\wedge}$. Then $H$ is compact and $G / H \cong L^{\wedge}$, which, by Corollary 1 of Theorem 2.5 , has no small subgroups, since $L$ is compactly generated. If $\xi$ is a character of $G^{\wedge} / L$, then $\xi \in G^{\wedge}=G$, and $\xi(L)=(0)$, so that $\xi(V)=(0) \subset \Gamma$. Hence $\xi \in U$. Thus $H \subset U$. 
Let $G$ and $H$ be in $\mathscr{L}$. We denote by $\operatorname{Hom}(G, H)$ the set of continuous homomorphisms from $G$ to $H$; by $\sum f^{\wedge}\left(H^{\wedge}\right)$ the subgroup of $G^{\wedge}$ generated by $f^{\wedge}\left(H^{\wedge}\right)$ for $f \in \operatorname{Hom}(G, H)$; and by $\bigcap \operatorname{Ker} f$ the intersection of $\operatorname{Ker} f$ for $f \in \operatorname{Hom}(G, H)$.

TheOREM 2.8. Let $G$ and $H$ be in $\mathscr{L}$. Then $\operatorname{Hom}(G, H)$ separates the points of $G$ if and only if $\sum f^{\wedge}\left(H^{\wedge}\right)$ is dense in $G^{\wedge}$.

Proof. Hom $(G, H)$ separates points of $G$ if and only if $\bigcap \operatorname{Ker} f=(0)$, that is, if and only if $\left(G^{\wedge}, \bigcap \operatorname{Ker} f\right)=\left(G^{\wedge},(0)\right)=G^{\wedge}$. But $\left(G^{\wedge}, \bigcap \operatorname{Ker} f\right)=\left(\sum\left(G^{\wedge}, \operatorname{Ker} f\right)\right)^{-}$ (see [2]). Hence $\operatorname{Hom}(G, H)$ separates the points of $G$ if and only if $\sum\left(G^{\wedge}, \operatorname{Ker} f\right)$ is dense in $G^{\wedge}$. However, $\left(G^{\wedge}, \operatorname{Ker} f\right)=\left(f^{\wedge}\left(H^{\wedge}\right)\right)^{-}$, by Proposition 2.1. The result follows if we can show that if $\sum\left(f^{\wedge}\left(H^{\wedge}\right)\right)^{-}$is dense in $G^{\wedge}$, then $\sum f^{\wedge}\left(H^{\wedge}\right)$ is dense in $G^{\wedge}$. Now let $\xi \in G^{\wedge}$ and $U$ be a neighborhood of 0 in $G^{\wedge}$. Choose $U_{1}$ so that $2 U_{1} \subset U$. There exists $\xi_{1}, \ldots, \xi_{n}$ so that $\xi_{i} \in f_{i}^{\wedge}\left(H^{\wedge}\right)^{-}$and $\left(\sum_{i=1}^{n} \xi_{i}\right)-\xi \in U_{1}$. Let $U_{2}$ be a neighborhood of 0 in $G^{\wedge}$ satisfying $n U_{2} \subset U_{1}$, and choose $\eta_{i} \in H^{\wedge}$ so that $f_{i}^{\wedge}\left(\eta_{i}\right)-\xi_{i} \in U_{2}, i=1, \ldots, n$. Then $\sum_{i} f_{i}^{\wedge}\left(\eta_{i}\right)-\xi \in 2 U_{1} \subset U$.

Corollary. Hom $(G, R)$ separates points of $G$ if and only if the set of points of $G^{\wedge}$ lying on one-parameter subgroups is dense in $G^{\wedge}$.

Proof. In particular, if we take $H=R$, then $R=R^{\wedge}$. It follows that $\sum f^{\wedge}\left(R^{\wedge}\right)$ equals the subgroup of $G^{\wedge}$ generated by the points which lie on one-parameter subgroups. Now, for any abelian topological group $A$,

$$
\sum\{f(R): f \in \operatorname{Hom}(R, A)\}=\bigcup\{f(R): f \in \operatorname{Hom}(R, A)\} .
$$

For suppose $x=\sum_{i=1}^{n} x_{i}$, where $x_{i} \in f_{i}(R)$; that is $x_{i}=f_{i}\left(t_{i}\right)$. We can assume $x_{i} \neq 0$ for all $i$. Choose a new parametrization, say $g_{i}$ of $f_{i}$ so that $x_{i}=g_{i}(1)$. Then $g(t)=\sum_{i=1}^{n} g_{i}(t)$ is a one-parameter subgroup of $A$, and $g(1)=x$.

We now study dual properties of compactly generated groups and groups without small subgroups, which generalize the usual facts about compact-discrete duality.

THEOREM 2.9. Let $G$ be a compactly generated group in $\mathscr{L}$ and $G^{\wedge}$ be its character group. Then (1) $G$ is torsion free if and only if $G^{\wedge}$ is divisible. (2) $G$ is divisible if and only if $G^{\wedge}$ is torsion free.

Proof. For each positive integer $n$, we define $f_{n}: G \rightarrow G$ by $f_{n}(x)=n x$. Clearly, $f_{n}$ is a continuous homomorphism. By Theorem 2.5, $G \cong R^{k} \oplus T^{m} \oplus C$, and evidently $f_{n}$ operates componentwise. Now $f_{n}\left(R^{k}\right)=R^{k}$, since vector groups are divisible. $f_{n}\left(Z^{m}\right)$ is closed in $Z^{m}$ since $Z^{m}$ is discrete; finally $f_{n}(C)$ is compact since $f_{n}$ is continuous. The product of closed sets is closed in the product topology; hence $f_{n}(G)=f_{n}\left(R^{k}\right) \oplus f_{n}\left(Z^{m}\right) \oplus f_{n}(C)$ is closed in $G$, and is therefore locally compact. The Open Mapping Theorem guarantees that $f_{n}$ is proper. By Proposition 2.2, $f_{n}$ is proper, and $f_{n}^{\wedge}\left(G^{\wedge}\right)=\left(G^{\wedge}, \operatorname{Ker} f_{n}\right)$. One computes easily that $f_{n}^{\wedge}(\xi)=n \xi$ for $\xi \in G^{\wedge}$. Hence $\left(G^{\wedge}, \operatorname{Ker} f_{n}\right)=n G^{\wedge}$. Now $n\left(G^{\wedge}\right)=G^{\wedge}$ if and only if $\operatorname{Ker} f_{n}=(0)$. 
Thus $G^{\wedge}$ is divisible if and only if $G$ is torsion free. Part 2 of this theorem follows in a similar way from $n(G)=\left(G, \operatorname{Ker} f_{n}^{\wedge}\right)$.

COROLlaRY. Let $G$ be a connected group in $\mathscr{L}$ and $H$ a closed pure subgroup of $G$. Then $H$ is connected.

Proof. By Corollary 2 of Theorem 2.5, $G^{\wedge}$ is torsion free. Hence by Theorem 2.9, $G$ is divisible. Now by definition, see [3], $n H=H \cap n G=H$ for each integer $n$. Thus $H$ is divisible. Since $H$ is compactly generated, by Theorem 2.6, it follows from Theorem 2.9 that $H^{\wedge}$ is torsion free and hence from Corollary 2 of Theorem 2.5 that $H$ is connected.

\section{Projectives and injectives.}

Definition. $I$ is an injective of $\mathscr{L}$ if $I$ is in $\mathscr{L}$, and for all short proper exact sequences and continuous homomorphisms $g: G_{1} \rightarrow I$ there is a continuous homomorphism $g^{-}: G_{2} \rightarrow I$ so that $g^{-} f_{1}=g$.

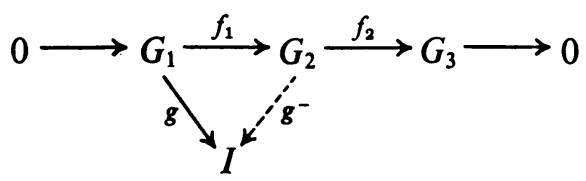

$P$ is a projective if $P$ is in $\mathscr{L}$, and for all short proper exact sequences and continuous homomorphisms $g: P \rightarrow G_{3}$ there is a continuous homomorphism $g^{-}: P \rightarrow G_{2}$ so that $f_{2} g^{-}=g$.

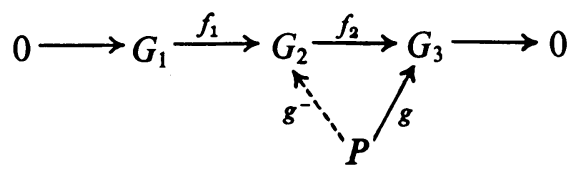

THEOREM 3.1. I is injective if and only if $I^{\wedge}$ is projective.

Proof. Suppose we are given

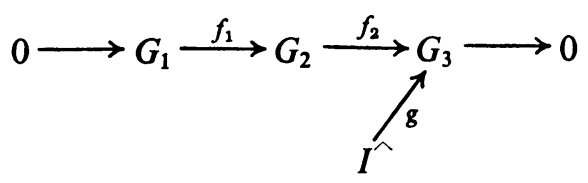

We dualize.

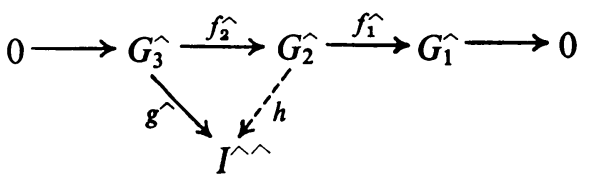

is exact.

Since $I^{\wedge \wedge}$ is injective there is a continuous homomorphism $h: G_{2}^{\wedge} \rightarrow I^{\wedge \wedge}$ such that $h f_{2}^{\wedge}=g^{\wedge}$. Thus $g=f_{2} h^{\wedge}$. So $h^{\wedge}$ is the required lift of $g$. The converse is proven similarly. 
Proposition 3.1. If $\left\{I_{a}: a \in A\right\}$ is a family of injectives of $\mathscr{L}$, such that $\prod_{a \in A} I_{a}$ is in $\mathscr{L}$ then $\prod_{a \in A} I_{a}$ is an injective of $\mathscr{L}$.

Proposition 3.2. Conversely, if $I=\prod_{a \in A} I_{a}$ and is injective, then $I_{a}$ is injective for all $a \in A$.

Propositions 3.1 and 3.2 follow immediately from the definitions.

Proposition 3.3. If I is injective for $\mathscr{L}$ then I is connected.

Proof. Consider the proper exact sequence $0 \rightarrow Z \rightarrow R \rightarrow T \rightarrow 0$. Let $y \in I$. Define a continuous homomorphism $f: Z \rightarrow I$ by $f(n)=n y$. Since $I$ is injective, $f$ extends to $f^{-}: R \rightarrow I$. Now $f^{-}(R)$ is connected and hence is contained in $I_{0}$. In particular $y \in I_{0}$. Thus $I \subset I_{0}$. In fact, each point of $I$ lies on a 1 parameter subgroup.

Proposition 3.4. If $I$ is in $\mathscr{L}$ and is injective for the class of compactly generated groups, then $I$ is injective for $\mathscr{L}$.

Proof. By considering the class of extensions $0 \rightarrow n Z \rightarrow Z \rightarrow Z_{n} \rightarrow 0$ whose groups are compactly generated, we conclude that $I$ is divisible. Let $G$ be in $\mathscr{L}$, $H$ be a closed subgroup of $G$, and $f: H \rightarrow I$ be a continuous homomorphism. We show $f$ extends to a continuous homomorphism $G \rightarrow I$. $G$ has an open, compactly generated subgroup, say $L$. By assumption, $\left.f\right|_{L \cap H}$ extends to a continuous homomorphism $f_{1}: L \rightarrow I$. Let $f_{2}$ be the homomorphism of the external direct $\operatorname{sum} L \oplus H$ into $I$, defined by $f_{2}((l, h))=f_{1}(l)+f(h)$. The kernel of the canonical epimorphism of $L \oplus H$ onto the subgroup $L+H$ of $G$ consists of the elements $(x,-x)$ with $x$ in $L \cap H$. Evidently these elements lie in the kernel of $f_{2}$. Hence $f_{2}$ induces a homomorphism $f_{3}$ of $L+H$ into $I$ such that $f_{3}(l+h)=f_{1}(l)+f(h)$. Moreover, $f_{3}$ extends $f_{1}$, since $f$ is a homomorphism. Since $L$ is open and $f_{1}$ is continuous on $L, f_{3}$ is continuous, because any homomorphic extension of a continuous homomorphism defined on an open subgroup is itself continuous. Since $I$ is divisible and $L+H$ is open, $f_{3}$ extends to a continuous homomorphism $f_{4}: G \rightarrow I$, and evidently $f_{4}$ extends $f$.

THEOREM 3.2. The following conditions on the locally compact abelian group I are equivalent.

(1) I is injective for the class of compactly generated groups,

(2) I is injective for $\mathscr{L}$,

(3) $I \cong R^{n} \oplus T^{\sigma}$, the direct product of a vector group and a (possibly infinitedimensional) torus $\left({ }^{4}\right)$.

Proof. If $I$ is injective, then by Proposition 3.3, $I=R^{n} \oplus C$ where $C$ is compact and connected. As a direct summand, $C$ is also injective, by Proposition 3.2. Hence, $C^{\wedge}$ is a discrete projective of $\mathscr{L}$, and is therefore a projective of $\mathscr{D}$, the

$\left.{ }^{4}\right)$ It was recently pointed out to me by Professor G. P. Hochschild that the fact that $R$ is injective was proven by J. Dixmier [7], for a different purpose. 
category of discrete abelian groups. As a projective of $\mathscr{D}, C^{\wedge}$ is a free abelian group, i.e., a direct sum of copies of $Z$. Hence $C$ is a direct product of copies of $T$.

Conversely, $R^{n} \oplus T^{\sigma}$ is injective for $\mathscr{L}$. In fact, by Proposition 3.1, it is sufficient to prove that $R$ and $T$ are injective. As is known (see [2]) $T$ is injective for $\mathscr{L}$. We now show that $R$ is injective for $\mathscr{L}$. It is sufficient to show that $R$ is injective for the class of compactly generated groups. Let $G_{2}$ be compactly generated and let $G_{1}$ be a closed subgroup of $G_{2}$. Since $G_{1}$ is compactly generated by Theorem 2.6, Theorem 2.5 shows that $G_{i} \cong W_{i} \oplus C_{i}$ where $W_{i}$ is the direct sum of a vector group and a discrete finitely generated free abelian group, and $C_{i}$ is a compact group for $i=1,2$. Since $W_{1}$ admits a topological embedding as a closed subgroup of a vector group, it follows that $W_{1}$ has no nontrivial compact subgroups because vector groups have this property. Let $\pi: G_{2} \rightarrow W_{2}$ be the canonical epimorphism with kernel $C_{2}$. Let $\alpha: W_{1} \rightarrow W_{2}$ be the restriction of $\pi$ to $W_{1}$. Then $\operatorname{Ker} \alpha$ $=W_{1} \cap C_{2}=(0)$ so that $\alpha$ is a continuous monomorphism. Since $C_{2}$ is compact, $\pi$ is a closed map (see [1]). Now $W_{1}$ is a closed subgroup of $G_{1}$, which is a closed subgroup of $G_{2}$. Hence $\pi\left(W_{1}\right)$ is closed in $W_{2}$ and therefore is locally compact. Since $W_{1}$ is compactly generated, $\alpha$ is a proper monomorphism, by the Open Mapping Theorem. In addition, since $C_{2}$ is the maximum compact subgroup of $G_{2}$, it follows that $C_{1} \subset C_{2}$.

Now let $f \in \operatorname{Hom}\left(G_{1}, R\right)$, and write $A$ for $\pi\left(W_{1}\right)$. Clearly, $f\left(C_{1}\right)=(0)$. Since $\alpha^{-1}: A \rightarrow W_{1}$ is a topological group isomorphism, it follows that

$$
\left(\left.f\right|_{W_{1}}\right) \circ \alpha^{-1} \in \operatorname{Hom}(A, R) \text {. }
$$

Suppose there exists $g \in \operatorname{Hom}\left(W_{2}, R\right)$ so that $\left.g\right|_{A}=\left(\left.f\right|_{W_{1}}\right) \circ \alpha^{-1}$. Then define $f^{-}: G_{2} \rightarrow R$ by $f^{-}(x+y)=g(x)$ where $x \in W_{2}$ and $y \in C_{2}$. Evidently $f^{-} \in \operatorname{Hom}\left(G_{2}, R\right)$. If $s+t \in G_{1}$ where $s \in W_{1}$ and $t \in C_{1}$, then $f(s+t)=f(s)+f(t)$ $=f(s)$, since $f\left(C_{1}\right)=(0)$. But $g(\alpha(s))=f(s)$, by the choice of $g$. Hence $f(s+t)=g(\alpha(s))$. On the other hand, $f^{-}(s+t)=f^{-}(s)+f^{-}(t)=f(s)$, since, clearly, $f^{-}\left(C_{1}\right)=(0)$. Now $s=x+y$, where $x \in W_{2}$ and $y \in C_{2}$. Therefore $f^{-}(s)=g(x)$, by the definition of $f^{-}$. But $x=\alpha(s)$. So $f^{-}(s)=g(\alpha(s))$. Therefore $f^{-}(s+t)=g(\alpha(s))=f(s+t)$ and $\left.f^{-}\right|_{G_{1}}=f$.

Thus we have reduced the problem to showing that if $W_{1}$ is a subgroup of $W_{2}$ ( $W_{i}$ as above), and $f \in \operatorname{Hom}\left(W_{1}, R\right)$ then there exists $g \in \operatorname{Hom}\left(W_{2}, R\right)$ so that $\left.g\right|_{W_{1}}=f$. Since one can embed $W_{2}$ as a closed subgroup of a vector group, say $V$, if $f$ can be extended to $g \in \operatorname{Hom}(V, R)$, then $\left.g\right|_{W_{2}}$ will be the desired extension of $f$. Thus we may assume that $W_{2}$ is a vector group. We have $W_{1}=V_{1} \oplus Z^{m_{1}}$, where $V_{1}$ is a vector group. Let $\left\{x_{1}, \ldots, x_{n}\right\}$ be a basis of $V_{1}$ as a vector space, and $\left\{y_{1}, \ldots, y_{k}\right\}$ a basis of $Z^{m_{1}}$ as a finitely generated free abelian group. Clearly $\left\{x_{1}, \ldots, x_{n}, y_{1}, \ldots, y_{k}\right\}$ are linearly independent in $V$ and can be extended to a basis of $V$, say $\left\{x_{1}, \ldots, x_{n}, y_{1}, \ldots, y_{k}, z_{1}, \ldots, z_{j}\right\}$. If

$$
x=\sum_{i=1}^{n} a_{i} x_{i}+\sum_{i=1}^{k} b_{i} y_{i}+\sum_{i=1}^{j} c_{i} z_{i}
$$


is in $V$, let $g$ be the linear (and hence continuous) functional defined by

$$
g(x)=\sum_{i=1}^{n} a_{i} f\left(x_{i}\right)+\sum_{i=1}^{k} b_{i} f\left(y_{i}\right)
$$

Since $f$ is a continuous homomorphism on $V_{1}, f$ is linear on $V_{1}$ and therefore $g$ extends $f$. This completes the proof that $R$ is injective. We have shown that (2) and (3) are equivalent. Proposition 3.4 completes the proof of Theorem 3.2.

Corollary 1. Let $G$ be in $\mathscr{L}$ and $H$ be a closed subgroup of $G$. Any one-parameter subgroup $f$ of $G / H$ lifts to a one-parameter subgroup $f^{-}$of $G$.

Proof. This follows directly from Theorem 3.2 and Theorem 3.1. The above corollary and our previous results yield an easy proof of the following known result.

Corollary 2. Let $G$ be in $\mathscr{L} . G$ is connected if and only if the set of points of $G$ which lie on one-parameter subgroups is dense in $G$.

Proof. Suppose that $G$ is connected. Let $U$ be any neighborhoood of 0 and let $x \in G$. By Theorem 2.7, choose a compact subgroup $H$ of $G$ so that $H \subset U$ and $G / H$ has no small subgroups. Denote the canonical epimorphism $G \rightarrow G / H$ by $\pi$. $G / H$ is connected and therefore is of the form $R^{n} \oplus T^{m}$. Clearly, each point of $G / H$ lies on a one-parameter subgroup. Hence there is an $f \in \operatorname{Hom}(R, G / H)$ with the property that $f(1)=\pi(x)$. By Corollary 1 of Theorem $3.2, f$ lifts to $f^{-} \in \operatorname{Hom}(R, G)$ : that is, $\pi f^{-}=f$. Hence, $\pi f^{-}(1)=f(1)=\pi(x)$, so that $f^{-}(1)-x \in H \subset U$. Thus, the set of points of $G$ that lie on one-parameter subgroups is dense in $G$. The converse is clear.

Corollary 3. Let $G$ be a locally compact abelian group. Hom $(G, R)$ separates the points of $G$ if and only if $G^{\wedge}$ is connected, i.e., if and only if $G=V \oplus D$, where $V$ is a vector group and $D$ is a torsion-free discrete group.

Proof. This follows directly from Corollary 2 of Theorem 3.2 and the Corollary to Theorem 2.8 .

THEOREM 3.3. The following conditions on a locally compact abelian group $P$ are equivalent.

(1) $P$ is projective for the class of groups without small subgroups.

(2) $P$ is projective for $\mathscr{L}$.

(3) $P \cong R^{n} \oplus \sum_{\sigma} Z$, the direct product of a vector group and a discrete free abelian group.

Proof. Theorem 3.3 is dual to Theorem 3.2.

CoRollaRy 1. If $G$ is a projective of $\mathscr{L}$ and $H$ is a closed subgroup of $G$, then $H$ is a projective of $\mathscr{L}$. 
Proof. By Theorem 3.3, it is sufficient to show $H$ is isomorphic to the direct product of a vector group and a discrete free abelian group. From Theorem 3.3 we know that $G=V \oplus D$, where $V$ is a vector group and $D$ is a discrete free abelian group. Then $H_{0} \subset G_{0}=V$, so $H_{0}$ is a closed connected subgroup of $V$. This implies that $H_{0}$ is itself a vector group and hence an injective for $\mathscr{L}$, so that $H_{0}$ is a direct factor of $H ; H \cong H_{0} \oplus H / H_{0}$. We may therefore assume, for the purpose of proving the corollary, that $H_{0}=(0)$. Now $G$ has no small subgroups. Hence, by Theorem 2.6, $H$ has no small subgroups. By Corollary 1 of Theorem 2.4, $H_{0}$ is open in $H$. Hence $H$ is discrete. Let $\pi$ be the canonical epimorphism $G \rightarrow D$ with kernel $V$. Then $\operatorname{Ker}\left(\left.\pi\right|_{H}\right)=H \cap V$, a discrete subgroup of a vector group, which is therefore free. Moreover, $\left.\pi\right|_{H}$ induces a monomorphism $H / H \cap V \rightarrow D$. Hence $H / H \cap V$ is isomorphic with a subgroup of a free group and is itself free. Let

$$
\gamma: H \rightarrow H / H \cap V
$$

be the canonical epimorphism, and consider $\operatorname{id}(H / H \cap V)$. Since $H / H \cap V$ is a discrete free abelian group it is a projective for $\mathscr{L}$, so that $\operatorname{id}(H / H \cap V)$ lifts to a (continuous) homomorphism $f: H / H \cap V \rightarrow H$ with the property that $\gamma f=\operatorname{id}(H / H \cap V)$. Hence $H \cong(H \cap V) \oplus(H / H \cap V)$. Since both $H \cap V$ and $H / H \cap V$ are free, $H$ is free.

COROLlary 2. Dually, proper homomorphic images of injectives are injectives, and in particular, the image under a continuous homomorphism of a torus is a torus. (Proper and continuous are equivalent here.)

The following characterization of vector groups illustrates their particular importance.

Corollary 3. A group $G$ is both projective and injective for $\mathscr{L}$ if and only if $G$ is a vector group.

Proof. If $G$ is projective then $G \cong V \oplus \sum_{\sigma} Z$, the direct product of a vector group and a discrete free abelian group. Since $G$ is injective it must be connected, hence $G=V$ a vector group. Conversely a vector group is both projective and injective.

THEOREM 3.4. $G_{1}$ is injective if and only if $G_{1}$ is compactly generated and every proper short exact sequence beginning with $G_{1}$ splits.

Proof. If $G_{1}$ is injective, then evidently every proper exact sequence

$$
0 \rightarrow G_{1} \rightarrow G_{2} \rightarrow G_{3} \rightarrow 0
$$

splits and, by Theorem 3.2, $G_{1}$ is compactly generated.

Now assume $G$ is compactly generated, and all short exact sequences beginning with $G$ split. We have $G \cong R^{n} \oplus Z^{m} \oplus C$, where $C$ is compact. Because the characters of $C$ separate points, $C$ can be embedded in $T^{\sigma(G)}$, via

$$
0 \longrightarrow C \stackrel{f_{1}}{\longrightarrow} T^{\sigma(G)}
$$


say. Since $f_{1}$ is a continuous monomorphism and $C$ is compact, $f_{1}$ is proper. If we denote by $f_{2}$ the canonical epimorphism of $T^{\sigma(G)} \rightarrow T^{\sigma(G)} / f_{1}(C)$, then

$$
0 \longrightarrow C \stackrel{f_{1}}{\longrightarrow} T^{\sigma(G)} \stackrel{f_{2}}{\longrightarrow} T^{\sigma(G)} / f_{1}(C) \longrightarrow 0
$$

is a proper short exact sequence. By Corollary 2 of the previous theorem, $T^{\sigma(G)} \mid f_{1}(C) \cong T^{\sigma^{\prime}(G)}$, a torus. Since $0 \rightarrow Z^{m} \rightarrow R^{m} \rightarrow T^{m} \rightarrow 0$ is a proper short exact sequence beginning with $Z^{m}$, and $0 \rightarrow R^{n} \rightarrow R^{n} \rightarrow 0 \rightarrow 0$ is a proper short exact sequence beginning with $R^{n}$, it follows directly that

$$
0 \rightarrow G \rightarrow R^{n+m} \oplus T^{\sigma(G)} \rightarrow T^{\sigma^{\prime}(G)+m} \rightarrow 0
$$

is a proper short exact sequence. This sequence splits, so $G$ is a direct summand of $R^{n+m} \oplus T^{\sigma(G)}$ which is injective. Hence $G$ is injective by Corollary 2 of Theorem 3.3.

COROLlaRY. If $G$ is in $\mathscr{L}$ then vector subgroups and toral subgroups of $G$ are direct summands.

THEOREM 3.5. A group $G_{3}$ is projective if and only if $G_{3}$ has no small subgroups, and every proper short exact sequence $0 \rightarrow G_{1} \rightarrow G_{2} \rightarrow G_{3} \rightarrow 0$ ending with $G_{3}$ splits.

Proof. Theorem 3.5 is dual to Theorem 3.4.

Definition. For a locally compact abelian group $G$ we say that a sequence of homomorphisms

$$
(0) \longrightarrow G \stackrel{e}{\longrightarrow} I_{0} \stackrel{d_{0}}{\longrightarrow} I_{1} \stackrel{d_{1}}{\longrightarrow} I_{2} \stackrel{d_{2}}{\longrightarrow} \cdots
$$

is an injective resolution of $G$ if each $I_{n}$ is injective and the sequence is proper exact. We say that a sequence of homomorphisms

$$
\cdots \longrightarrow P_{3} \stackrel{d_{2}}{\longrightarrow} P_{2} \stackrel{d_{1}}{\longrightarrow} P_{1} \stackrel{d_{0}}{\longrightarrow} P_{0} \stackrel{\pi}{\longrightarrow} G \longrightarrow(0)
$$

is a projective resolution of $G$ if each $P_{n}$ is projective and the sequence is proper exact.

Definition. If $I_{n}=(0), n \geqq 2$ or $P_{n}=(0), n \geqq 2$, we call the resolution short.

THEOREM 3.6. (1) $G$ has an injective resolution if and only if $G$ is compactly generated, and in that case there is a short injective resolution.

(2) $G$ has a projective resolution if and only if $G$ has no small subgroups, and in that case there is a short projective resolution.

Proof. Let $G$ be compactly generated. In the course of proving Theorem 3.4, we constructed the proper short exact sequence $0 \rightarrow G \rightarrow R^{n+m} \oplus T^{\sigma} \rightarrow T^{\sigma^{\prime}} \rightarrow 0$. This is a short injective reolution.

Conversely, if $G \in \mathscr{L}$ and has an injective resolution

$$
0 \longrightarrow G \stackrel{e}{\longrightarrow} I_{0} \stackrel{d_{0}}{\longrightarrow} I_{1} \stackrel{d_{1}}{\longrightarrow} I_{2} \stackrel{d_{2}}{\longrightarrow} \cdots,
$$


then $e(G) \cong G$. But $e(G)$ is locally compact and therefore a closed subgroup of $I_{0}$. Since $I_{0}$ is injective it is compactly generated. Hence $e(G)$, and therefore $G$, is compactly generated.

(2) follows from (1) by dualization. The above injective resolution and its dual are clearly the ones to compute with.

IV. Multilinear functions and tensor products in commutative topological groups. Let $G_{1}, \ldots, G_{r}$ and $G$ be commutative topological groups. We call a map $\mu: \prod_{i=1}^{r} G_{i} \rightarrow G r$-linear if for all $x_{i}$ and $y_{i}$ in $G_{i}(i=1, \ldots, r), \mu\left(x_{1}, \ldots, x_{i}+y_{i}, \ldots, x_{r}\right)$ $=\mu\left(x_{1}, \ldots, x_{i}, \ldots, x_{r}\right)+\mu\left(x_{i}, \ldots, y_{i}, \ldots, x_{r}\right)$. We denote by $M_{G}\left(G_{1}, \ldots, G_{r}\right)$ the set of all $r$ linear continuous $G$ valued maps on $\prod_{i=1}^{r} G_{i}\left({ }^{5}\right) . M_{G}\left(G_{1}, \ldots, G_{r}\right)$ becomes a commutative group under pointwise addition.

We topologize $M_{G}\left(G_{1}, \ldots, G_{r}\right)$ by uniform convergence on compact subsets. More precisely, for each compact subset $F$ of $\prod_{i=1}^{r} G_{i}$, and for each neighborhood $\Gamma$ of 0 in $G$ we define $W(F, \Gamma)$ to be the set $\left\{\mu: \mu \in M_{G}\left(G_{1}, \ldots, G_{r}\right)\right.$ and $\left.\mu(F) \subset \Gamma\right\}$. It is easily verified that, with this family of sets as a fundamental system of neighborhoods of $0, M_{G}\left(G_{1}, \ldots, G_{r}\right)$ is a topological group.

THEOREM 4.1. (1) If $G_{1}, \ldots, G_{r}$ are compactly generated and $G$ is compact without small subgroups, then $M_{G}\left(G_{1}, \ldots, G_{r}\right)$ is locally compact.

(2) If $G_{1}, \ldots, G_{r}$ are compact and $G$ is without small subgroups, then $M_{G}\left(G_{1}, \ldots, G_{r}\right)$ is discrete.

(3) If $G_{1}, \ldots, G_{r}$ are discrete and $G$ is compact, then $M_{G}\left(G_{1}, \ldots, G_{r}\right)$ is compact.

Proof. (1) $\prod_{i=1}^{r} G_{i}$ is clearly locally compact. The topology on the function space $M_{G}\left(G_{1}, \ldots, G_{r}\right)$ is the compact-open topology. In this setting the Ascoli Theorem states that a subset $F$ of the space of all continuous maps $\prod_{i=1}^{r} G_{i} \rightarrow G$, with the compact-open topology, is compact if and only if (a) $F$ is closed, (b) for each $x \in \prod_{i=1}^{r} G_{i}$, the set $\{f(x): f \in F\}$ has compact closure in $G$, and (c) $F$ is equicontinuous at each point $x$ of $\prod_{i=1}^{r} G_{i}$.

For each $i=1, \ldots, r$ let $U_{i}$ be a compact neighborhood of 0 generating $G_{i}$. We denote $\prod_{i=1}^{r} U_{i}$ by $U$. Since $G$ has no small subgroups, it possesses a closed test neighborhood of 0 , say $\Gamma_{*}$. Thus $W\left(U, \Gamma_{*}\right)$ is a neighborhood of 0 in $M_{G}\left(G_{1}, \ldots, G_{r}\right)$. We show that $W\left(U, \Gamma_{*}\right)$ is compact.

Since $\Gamma_{*}$ is a closed neighborhood, it is clear that $W\left(U, \Gamma_{*}\right)$ is closed. $W\left(U, \Gamma_{*}\right)$ satisfies condition (b) automatically, since $G$ is compact.

Now we show that $W\left(U, \Gamma_{*}\right)$ is equicontinuous at $x$, where $x=\left(x_{1}, \ldots, x_{r}\right)$. For each $i=1, \ldots, r, x_{i}=\sum_{l=1}^{n_{i}} x_{i_{l}}$ where $n_{i}$ is an integer and $x_{i_{l}} \in U_{i}$. We denote $\max \left\{n_{i}: i=1, \ldots, r\right\}$ by $n(x)$. If $\Gamma$ is any neighborhood of 0 in $G$, let $\Gamma^{\prime}$ be a neighborhood of 0 in $G$ with the property that $\left(2^{r}-1\right) \Gamma^{\prime} \subset \Gamma$. Choose $\Gamma^{\prime \prime}$ small enough so that $(r-1) n(x) \Gamma^{\prime \prime} \subset \Gamma^{\prime}$, and $k\left(\Gamma^{\prime \prime}\right)$ as usual. Finally, for each $i=1, \ldots, r$, choose a neighborhood of $0, V_{i}$, in $G_{i}$ with the property that $k\left(\Gamma^{\prime \prime}\right) V_{i} \subset U_{i}$. Let $V(x, \Gamma)$

(5) We note that for $r>1$ continuity at 0 does not guarantee continuity everywhere. 
$=\prod_{i=1}^{r} V_{i}$. This is a neighborhood of 0 in $\prod_{i=1}^{r} G_{i}$. We show that $\mu(x+V(x, \Gamma))$ $\subset \mu(x)+\Gamma$ for every $\mu \in W\left(U, \Gamma_{*}\right)$.

For each $\left(v_{1}, \ldots, v_{r}\right) \in V$,

$$
\mu\left(x_{1}+v_{1}, \ldots, x_{r}+v_{r}\right)=\mu\left(x_{1}, \ldots, x_{r}\right)+\sum_{2^{r}-2}+\mu\left(v_{1}, \ldots, v_{r}\right) .
$$

Since $\left(2^{r}-1\right) \Gamma^{\prime} \subset \Gamma$, it is sufficient to show that $\mu\left(v_{1}, \ldots, v_{r}\right)$ and each of the terms in $\sum_{2^{r}-2}$ belong to $\Gamma^{\prime}$ for every $\mu \in W\left(U, \Gamma_{*}\right)$. First we observe that

$$
\mu\left(U_{1} \times \cdots \times V_{i} \times \cdots \times U_{r}\right) \subset \Gamma^{\prime \prime}
$$

for each $\mu \in W\left(U, \Gamma_{*}\right)$ and each $i=1, \ldots, r$, since

$$
j \mu\left(u_{1}, \ldots, v_{i}, \ldots, u_{r}\right)=\mu\left(u_{1}, \ldots, j v_{i}, \ldots, u_{r}\right) \in \mu(U) \subset \Gamma_{*},
$$

for all $j=1, \ldots, k\left(\Gamma^{\prime \prime}\right)$. In particular, $\mu\left(V_{1} \times \cdots \times V_{r}\right) \subset \Gamma^{\prime}$. Also, for each $q=1, \ldots$, $r-1$,

$$
\begin{aligned}
\mu\left(v_{1}, \ldots, v_{q}, x_{q+1}, \ldots, x_{r}\right)=\mu\left(v_{1}, \ldots, v_{q}, \sum_{l=1}^{n(x)} x_{q+1 l_{q+1}}, \ldots, \sum_{l=1}^{n(x)} x_{r l_{r}}\right) \\
=\sum_{l_{q+1}, \ldots, l_{r}=1}^{n(x)} \mu\left(v_{1}, \ldots, v_{q}, x_{q+1 l_{q+1}}, \ldots, x_{r l_{r}}\right) \in(r-1) n(x) \Gamma^{\prime \prime} \subset \Gamma^{\prime}
\end{aligned}
$$

and we may deal similarly with the other terms of $\sum_{2^{r}-2}$. This completes the proof of part 1.

(2) Let $\Gamma_{*}$ be a neighborhood of 0 in $G$ which contains no nontrivial subgroups. Since $\prod_{i=1}^{r} G_{i}$ is compact, $W\left(\prod_{i=1}^{r} G_{i}, \Gamma_{*}\right)$ is a neighborhood of 0 in $M_{G}\left(G_{1}, \ldots, G_{r}\right)$. Let $\mu \in W\left(\prod_{i=1}^{r} G_{i}, \Gamma_{*}\right)$ and suppose that $\mu\left(x_{1}, \ldots, x_{r}\right) \neq 0$ for some $\left(x_{1}, \ldots, x_{r}\right) \in \prod_{i=1}^{r} G_{i}$. Then $\mu\left(G_{1} \times\left\{x_{2}\right\} \times \cdots \times\left\{x_{r}\right\}\right)$ is a nontrivial homomorphic image of $G_{1}$ and is contained in $\Gamma_{*}$; a contradiction. Thus

$$
W\left(\prod_{i=1}^{r} G_{i}, \Gamma_{*}\right)=(0)
$$

and $M_{G}\left(G_{1}, \ldots, G_{r}\right)$ is discrete.

(3) $\prod_{i=1}^{r} G_{i}$ is clearly discrete and hence the compact subsets $F$ of $\prod_{i=1}^{r} G_{i}$ are finite. This means that $M_{G}\left(G_{1}, \ldots, G_{r}\right)$ is a subspace of $G \prod_{1}^{T} G_{t}$ with the product topology. $G$ is compact, so by the Tychonoff Theorem, $G^{\prod_{1}^{r} G_{t}}$ is also compact. We show that $M_{G}\left(G_{1}, \ldots, G_{r}\right)$ is closed in $G \prod_{1}^{r} G_{i}$. Let $\left\{\mu_{a}: a \in A\right\}$ be a net in $M_{G}\left(G_{1}, \ldots, G_{r}\right)$ which converges to a map $f: \prod_{1}^{r} G_{i} \rightarrow G$. If $x_{i}$ and $y_{i}$ belong to $G_{i}$, where $i$ is any integer between 1 and $r$, then

$$
\begin{gathered}
\mu_{a}\left(x_{1}, \ldots, x_{i}+y_{i}, \ldots, x_{r}\right) \underset{a}{\longrightarrow} f\left(x_{1}, \ldots, x_{i}+y_{i}, \ldots, x_{r}\right), \\
\mu_{a}\left(x_{1}, \ldots, x_{i}, \ldots, x_{r}\right) \underset{a}{\longrightarrow} f\left(x_{1}, \ldots, x_{i}, \ldots, x_{r}\right), \\
\mu_{a}\left(x_{1}, \ldots, y_{i}, \ldots, x_{r}\right) \underset{a}{\longrightarrow} f\left(x_{1}, \ldots, y_{i}, \ldots, x_{r}\right),
\end{gathered}
$$


by definition of the product topology. Because of the continuity of the group operation in $G$ and the fact that each $\mu_{a}$ is $r$ linear, it is clear that $f$ is $r$ linear. Since $\prod_{1}^{r} G_{i}$ is discrete, $f \in M_{G}\left(G_{1}, \ldots, G_{r}\right)$. This completes the proof.

If $r=1$ we write $\operatorname{Hom}\left(G_{1}, G\right)$ for $M_{G}\left(G_{1}, \ldots, G_{r}\right)$. In this case, we observe that equicontinuity at 0 implies equicontinuity at every point. Let $U_{1}$ be any compact neighborhood of 0 in $G_{1}$ and $\Gamma_{*}$ as above. We show that $W\left(U_{1}, \Gamma_{*}\right)$ is equicontinuous at 0 and is therefore compact. If $\Gamma$ is any neighborhood of 0 , choose $V$ so that $k(\Gamma) V \subset U_{1}$. Then for $v \in V$ and $h \in W\left(U_{1}, \Gamma_{*}\right)$,

$$
j h(v)=h(j v) \in h\left(U_{1}\right) \subset \Gamma_{*},
$$

for $j=1, \ldots, k(\Gamma)$. Therefore, $h(v) \in \Gamma$. Thus we have the following result.

COROLLARY. If $G_{1}$ is locally compact, and $G$ is compact without small subgroups, then $\operatorname{Hom}\left(G_{1}, G\right)$ is locally compact.

THeOREM 4.2. Let $G_{1}, \ldots, G_{r}$ be locally compact abelian groups, and $G$ be an arbitrary abelian topological group. For $r>1, M_{G}\left(G_{1}, \ldots, G_{r}\right)$ is isomorphic with Hom $\left(G_{1}, M_{G}\left(G_{2}, \ldots, G_{r}\right)\right)$ as a topological group.

Proof. If $S$ and $T$ are topological spaces we denote the set of all continuous maps $S \rightarrow T$ by $\mathscr{M}(S, T)$. If $\mathscr{M}(S, T)$ is regarded as a topological space with the compact open topology we write $\mathscr{M}^{*}(S, T)$ for this. A well known property of the compact open topologv is the following: if $S$ and $T$ are locally compact spaces and $X$ an arbitrary space, then the map $\tau: \mathscr{M}(S \times T, X) \rightarrow \mathscr{M}\left(S, \mathscr{M}^{*}(T, X)\right)$ defined by $\tau(f)(s)(t)=f(s, t)$ for $f \in \mathscr{M}(S \times T, X)$ and $(s, t) \in S \times T$, is a homeomorphism of $\mathscr{M}^{*}(S \times T, X)$ onto $\mathscr{M}^{*}\left(S, \mathscr{M}^{*}(T, X)\right)$. Now, $M_{G}\left(G_{1}, \ldots, G_{r}\right)$ and

$$
\operatorname{Hom}\left(G_{1}, M_{G}\left(G_{2}, \ldots, G_{r}\right)\right)
$$

are subspaces of $\mathscr{M}^{*}\left(\prod_{i=1}^{r} G_{i}, G\right)$ and $\mathscr{M}^{*}\left(G_{1}, M_{G}\left(G_{2}, \ldots, G_{r}\right)\right)$ respectively. The latter can be identified with a subspace of $\mathscr{M}^{*}\left(G_{1}, \mathscr{M}^{*}\left(\prod_{i=2}^{r} G_{i}, G\right)\right)$. The map $\tau$ sends the first homeomorphically onto the second and is evidently a group homomorphism.

COROLlARY 1. If $G_{1}$ is compactly generated and $G_{2}$ has no small subgroups, then Hom $\left(G_{1}, G_{2}\right)$ is locally compact.

Proof. By Theorem 4.2, $M_{G}\left(G_{1}, G_{2}\right) \cong \operatorname{Hom}\left(G_{1}\right.$, Hom $\left.\left(G_{2}, G\right)\right)$. Specializing to $G=T$ we find that $M_{T}\left(G_{1}, G_{2}^{\wedge}\right) \cong \operatorname{Hom}\left(G_{1}, G_{2}\right)$. Since $G_{2}$ has no small subgroups, $G_{2}^{\wedge}$ is compactly generated and, since $T$ is compact without small subgroups, Theorem 4.1 applies.

COROLlaRY 2. If $G_{1}$ and $G_{2}$ are any locally compact abelian groups then $\operatorname{Hom}\left(G_{1}, G_{2}\right)$ is isomorphic with $\operatorname{Hom}\left(G_{2}, G_{1}\right)$.

Proof. As we have already seen, $M_{T}\left(G_{1}, G_{2}^{\wedge}\right) \cong \operatorname{Hom}\left(G_{1}, G_{2}\right)$. Now $M_{T}\left(G_{1}, G_{2}\right)$ $\cong M_{T}\left(G_{2}, G_{1}\right)$, and the result follows by applying the same isomorphism with $\left(G_{2}, G_{1}^{\wedge}\right)$ in the place of $\left(G_{1}, G_{2}\right)$. 
The following classes of groups are dual to one another: locally compact groups are self dual; compactly generated groups are dual to the groups without small subgroups; discrete finitely generated groups are dual to compact groups without small subgroups. As a result of these facts, and of Corollary 2, theorems of the sort considered below can be immediately dualized. For example, we have shown that

(1) If $G$ is compactly generated and $H$ has no small subgroups then $\operatorname{Hom}(G, H)$ is locally compact.

(2) If $G$ is any locally compact group and $H$ is compact without small subgroups then $\operatorname{Hom}(G, H)$ is locally compact.

Statement (1) is self dual whereas statement (2) dualizes as follows. If $G$ is a finitely generated discrete group and $H$ is any locally compact group then Hom $(G, H)$ is locally compact. We show that, in a sense, these are the best possible results.

THEOREM 4.3. ( $\left(1^{\prime}\right)$ If $\operatorname{Hom}(G, H)$ is locally compact for all $H$ without small subgroups then $G$ is compactly generated.

(2') If Hom $(G, H)$ is locally compact for all $H$ then $G$ is a finitely generated discrete group.

Dually, $\left(1^{\prime \prime}\right)$ If Hom $(G, H)$ is locally compact for all compactly generated groups $G$ then $H$ is without small subgroups.

(2") If Hom $(G, H)$ is locally compact for all $G$ then $H$ is compact without small subgroups.

Proof. It is sufficient to prove $\left(1^{\prime}\right)$ and $\left(2^{\prime}\right)$.

(1') We actually prove a stronger result; namely, that if $\operatorname{Hom}(G, D)$ is locally compact for a certain fixed discrete group then $G$ is compactly generated.

For the moment, let $D$ denote an arbitrary discrete group. We may assume that Hom $(G, D)$ has a compact neighborhood of 0 of the form $W(F, \Gamma)$ where $F$ is a compact subset of $G$ and $\Gamma$ is a closed neighborhood of 0 in $D$. Since $D$ is discrete we may assume $\Gamma=(0)$. By a compactness argument, $F$ is contained in a compact neighborhood $U$ of 0 in $G$, which we may assume is symmetric. $W(U,(0))$ is a closed neighborhood of 0 in $\operatorname{Hom}(G, D)$ which is contained in $W(F,(0))$. Hence $W(U,(0))$ is compact. By the Ascoli Theorem, for each $x \in G$, $\{f(x): f \in W(U,(0))\}$ has compact closure in $D$, that is $\{f(x): f(U)=(0)\}$ is finite. Let $L=\bigcup_{n=1}^{\infty} n U$. Then $L$ is an open compactly generated subgroup of $G$. It is clear that for each $x \in G,\{f(x): f(L)=(0)\}$ is finite. Now take $D$ to be the direct sum of infinitely many copies of the additive group of the rationals mod 1 . We show that $G=L$.

Suppose $x \in G$ but $x \notin L$. Since $D$ is divisible, the 0 -map, $L \rightarrow(0)$, can be extended to a homomorphism $f: G \rightarrow D$ with the property that $f(x) \neq 0$. Since $L$ is open in $G, f$ is continuous. Furthermore, because of the choice of $D$, there are infinitely many such extensions all differing in value at $x$. For if $m x \in L$ for some 
integer $m$, any choice of $f(x)$ subject to the condition order $(f(x)) \mid m$ yields an extension of the sort described above, and there are infinitely many summands. On the other hand if $m x$ is never in $L, f(x)$ is completely arbitrary. Hence, $\{f(x): f(L)=(0)\}$ is infinite. This contradiction completes the proof of $\left(1^{\prime}\right)$.

(2') If $H$ ranges over all the groups without small subgroups then by (1') $G$ is compactly generated. Now take $H=T^{\sigma}$, any infinite-dimensional torus. We make use of the evident result that if $G$ is any abelian topological group and $\left\{H_{a}: a \in A\right\}$ is a family of abelian topological groups, then

$$
\operatorname{Hom}\left(G, \prod_{a \in A} H_{a}\right) \cong \prod_{a \in A} \operatorname{Hom}\left(G, H_{a}\right) \text {. }
$$

We have therefore, $\operatorname{Hom}(G, H) \cong \prod_{\sigma} \operatorname{Hom}(G, T)=\prod_{\sigma} G^{\wedge}$. Thus $\Pi_{\sigma} G^{\wedge}$ is locally compact. Hence all but a finite number of the factors are compact, so that $G^{\wedge}$ is compact and $G$ is discrete. Since $G$ is compactly generated and discrete, it is finitely generated.

Definition. If $G_{1}, \ldots, G_{r}$ is a family of locally compact abelian groups we define $\otimes_{i=1}^{r} G_{i}=M_{T}\left(G_{1}, \ldots, G_{r}\right)^{\wedge}$.

Notice that for any locally compact abelian group $G$ since $M_{T}(Z, G)$ is naturally isomorphic with $\operatorname{Hom}(G, T)$ by Theorem 4.2 , it follows that $Z \otimes G$ is naturally isomorphic with $G$.

THEOREM 4.4. $\bigotimes_{i=1}^{r} G_{i}$ is an abelian topological group. If $G_{1}, \ldots, G_{r}$ are compactly generated, compact, or discrete, then $\bigotimes_{i=1}^{r} G_{i}$ is locally compact, compact, or discrete, respectively $\left({ }^{6}\right)$.

Proof. This follows directly from Theorem 4.1.

Definition. We define the tensor map $\phi: \prod_{i=1}^{r} G_{i} \rightarrow \bigotimes_{i=1}^{r} G_{i}$ by

$$
\phi\left(x_{1}, \ldots, x_{r}\right)(\mu)=\mu\left(x_{1}, \ldots, x_{r}\right)
$$

for all $\mu \in M_{T}\left(G_{1}, \ldots, G_{r}\right)$. It is clear that $\phi\left(x_{1}, \ldots, x_{r}\right)$ is a continuous linear map $M_{T}\left(G_{1}, \ldots, G_{r}\right) \rightarrow T$, and thus a character of $M_{T}\left(G_{1}, \ldots, G_{r}\right)$.

Now let $D$ be the subgroup of $\bigotimes_{i=1}^{r} G_{i}$ that is generated by $\phi\left(G_{1} \times \cdots \times G_{r}\right)$. Obviously, $D$ is the set of all finite sums of elements of the form $\phi\left(x_{1}, \ldots, x_{r}\right)$.

THEOREM 4.5. (1) $\phi$ is a continuous $r$-linear map.

(2) If $M_{T}\left(G_{1}, \ldots, G_{r}\right)$ is locally compact $\left({ }^{7}\right)$ then $D$ is dense in $\bigotimes_{i=1}^{r} G_{i}$. In particular, if the $G_{i}$ are discrete then $D=\bigotimes_{i=1}^{r} G_{i}$.

Proof. $\phi$ is $r$-linear, because each of the $\mu \in M_{T}\left(G_{1}, \ldots, G_{r}\right)$ is $r$-linear. To show $\phi$ is continuous at $x$ it is sufficient to take a neighborhood $\phi(x)+W(F, \Gamma)$ of $\phi(x)$

$\left(^{6}\right)$ Actually, in $\S \mathrm{V}$ we shall see that if $G_{1}, \ldots, G_{r}$ are compactly generated then $\otimes_{i=1}^{r} G_{t}$ is also compactly generated.

$\left.{ }^{7}\right)$ Whenever we make the convenient hypothesis that $M_{T}\left(G_{1}, \ldots, G_{r}\right)$ is locally compact we really have in mind more accessible conditions; namely, that either the $G_{t}$ are compactly generated (Theorem 4.1, Part 1) or they are discrete (Theorem 4.1, Part 3). 
with $F$ a compact subset of $M_{T}\left(G_{1}, \ldots, G_{r}\right)$ and $\Gamma$ a neighborhood of 0 in $T$, and find a neighborhood $U(F, \Gamma)$ of $x$ in $\prod_{i=1}^{r} G_{i}$ so that $\phi(U) \subset \phi(x)+W(F, \Gamma)$, that is, $\mu(U) \subset \mu(x)+\Gamma$ for all $\mu \in F$. Thus the continuity of $\phi$ at $x$ is equivalent to the equicontinuity of $F$ at $x$. However, since $F$ is compact, the Ascoli Theorem guarantees that $F$ is equicontinuous at every point. Thus $\phi$ is continuous.

It is clear that $\mu^{\wedge \wedge}\left(\phi\left(x_{1}, \ldots, x_{r}\right)\right)=\phi\left(x_{1}, \ldots, x_{r}\right)(\mu)=\mu\left(x_{1}, \ldots, x_{r}\right)$. Hence if $\mu^{\wedge \wedge}$ annihilates $\phi\left(\prod_{1}^{r} G_{i}\right)$ then $\mu=0$, and so $\mu^{\wedge}$ is 0 because $\wedge$ is an isomorphism $M_{T}\left(G_{1}, \ldots, G_{r}\right) \rightarrow M_{T}\left(G_{1}, \ldots, G_{r}\right)^{\wedge \wedge}$. Consequently the annihilator of $D$ in $M_{T}\left(G_{1}, \ldots, G_{r}\right)^{\wedge \wedge}$ is $(0)$. Since $M_{T}\left(G_{1}, \ldots, G_{r}\right)$ is a locally compact abelian group, the result follows.

The following lemma is a known fact about the compact open topology.

LEMMA. If $T$ is a locally compact space, then the composition map

is continuous.

$$
\mathscr{M}^{*}(T, X) \times \mathscr{M}^{*}(S, T) \rightarrow \mathscr{M}^{*}(S, X)
$$

Now let $G$ and $H$ be in $\mathscr{L}$. It follows from the above lemma that the composition map $\operatorname{Hom}(G, H) \times M_{G}\left(G_{1}, \ldots, G_{r}\right) \rightarrow M_{H}\left(G_{1}, \ldots, G_{r}\right)$ is continuous. In particular for each $\mu$ in $M_{G}\left(G_{1}, \ldots, G_{r}\right)$, we define the map $\rho_{\mu}: G^{\wedge} \rightarrow M_{T}\left(G_{1}, \ldots, G_{r}\right)$ by $\rho_{\mu}(\xi)=\xi \circ \mu$ for $\xi \in G^{\wedge}$. Clearly, $\rho_{\mu}$ is a continuous homomorphism. Furthermore, it follows from the definitions of the maps involved that for each $\mu$ in $M_{G}\left(G_{1}, \ldots, G_{r}\right)$ we have $\hat{\rho_{\mu}} \circ \phi=\omega_{G} \circ \mu$, where $\omega_{G}$ is the natural isomorphism of $G$ onto $G^{\wedge}$.

TheOREM 4.6. Let $G$ and $G_{1}, \ldots, G_{r}$ be in $\mathscr{L}$. To each $\mu \in M_{G}\left(G_{1}, \ldots, G_{r}\right)$ there corresponds a $\chi_{\mu} \in \operatorname{Hom}\left(\bigotimes_{i=1}^{r} G_{i}, G\right)$ satisfying $\chi_{\mu} \phi=\mu$. If $M_{T}\left(G_{1}, \ldots, G_{r}\right)$ is locally compact, then the condition $\chi_{\mu} \phi=\mu$ characterizes $\chi_{\mu}$ uniquely.

Proof. Define $\chi_{\mu}=\omega_{G}^{-1} \hat{\rho_{\mu}}$ where $\omega_{G}$ and $\rho_{\mu}$ are as above. Then $\chi_{\mu}$ is a continuous homomorphism $\bigotimes_{i=1}^{r} G_{i} \rightarrow G$, since $\omega_{G}^{-1}$ and $\rho_{\mu}$ are continuous homomorphisms. Thus $\chi_{\mu} \in \operatorname{Hom}\left(\bigotimes_{i=1}^{r} G_{i}, G\right)$. Moreover, we have $\chi_{\mu} \phi=\omega_{G}^{-1} \widehat{\rho_{\mu}} \phi=\omega_{G}^{-1} \omega_{G} \mu=\mu$.

If $f_{\mu}$ is another continuous homomorphism satisfying $f_{\mu} \phi=\mu$, then, by linearity, $\left.f_{\mu}\right|_{D}=\left.\chi_{\mu}\right|_{D}$. Since $f_{\mu}$ and $\chi_{\mu}$ are both continuous and $D$ is dense, it follows that $f_{\mu}=\chi_{\mu}$. This completes the proof.

Denote by $\mathscr{A}$ the category of all abelian topological groups. We define two functors $\mathscr{F}, \mathscr{G}: \mathscr{L} \rightarrow \mathscr{A}$ by $\mathscr{F}(G)=M_{G}\left(G_{1}, \ldots, G_{r}\right), \mathscr{G}(G)=\operatorname{Hom}\left(\bigotimes_{i=1}^{r} G_{i}, G\right)$. If $G$ and $H$ are in $\mathscr{L}$ and $h$ is a continuous homomorphism then we define $\mathscr{F}(h)(\mu)=h \circ \mu$ for $\mu \in M_{G}\left(G_{1}, \ldots, G_{r}\right)$ and $\mathscr{G}(h)(f)=h \circ f$, for $f \in \operatorname{Hom}\left(\bigotimes_{i=1}^{r} G_{i}, G\right)$. It is clear that $\mathscr{F}$ and $\mathscr{G}$ are additive covariant functors.

THEOREM 4.7. If $M_{T}\left(G_{1}, \ldots, G_{r}\right)$ is locally compact then the functors $\mathscr{F}$ and $\mathscr{G}$ are isomorphic.

Proof. We denote the map $\mu \rightarrow \chi_{\mu}$ defined above by

$$
\chi: M_{G}\left(G_{1}, \ldots, G_{r}\right) \rightarrow \operatorname{Hom}\left(\bigotimes_{i=1}^{r} G_{i}, G\right) .
$$


If $f \in \operatorname{Hom}\left(\bigotimes_{i=1}^{r} G_{i}, G\right)$ then $f \circ \phi \in M_{G}\left(G_{1}, \ldots, G_{r}\right)$. Since $f$ and $\chi_{f \circ \phi}$ are continuous homomorphisms that coincide on $D$, we have $f=\chi_{f \circ \phi}$. On the other hand, if $\chi_{\mu}=\chi_{\nu}$ then $\chi_{\mu} \phi=\chi_{\nu} \phi$, i.e., $\mu=\nu$. Thus $\chi$ is a bijection.

We denote by $\rho$ the map $\mu \rightarrow \rho_{\mu}$. Thus

$$
\rho: M_{G}\left(G_{1}, \ldots, G_{r}\right) \rightarrow \operatorname{Hom}\left(G^{\wedge}, M_{T}\left(G_{1}, \ldots, G_{r}\right)\right) .
$$

One checks easily that $\rho$ is a continuous homomorphism.

Now consider the dualization map

$$
\lambda: \operatorname{Hom}\left(G^{\wedge}, M_{T}\left(G_{1}, \ldots, G_{r}\right)\right) \rightarrow \operatorname{Hom}\left(\bigotimes_{i=1}^{r} G_{i}, G\right)
$$

defined by $\lambda(h)=\omega_{G}^{-1} h^{\wedge}$, for $h \in \operatorname{Hom}\left(G^{\wedge}, M_{T}\left(G_{1}, \ldots, G_{r}\right)\right)$, where $\omega_{G}$ is the natural isomorphism of $G \rightarrow G^{\wedge}$. By Corollary 2, Theorem 4.2, the dualization map $\operatorname{Hom}(A, B) \rightarrow \operatorname{Hom}\left(B^{\wedge}, A^{\wedge}\right)$ is continuous. Hence $\lambda$ is continuous. As is easily seen, $\chi=\lambda \rho$, so that $\chi$ is a continuous homomorphism. If $W(F, \Gamma)$ is a neighborhood of 0 in $M_{G}\left(G_{1}, \ldots, G_{r}\right)$, since the tensor map $\phi$ is continuous, $\phi(F)$ is compact. Hence $W(\phi(F), \Gamma)$ is a neighborhood of 0 in $\operatorname{Hom}\left(\bigotimes_{i=1}^{r} G_{i}, G\right)$ which is contained in $\chi(W(F, \Gamma))$. Thus $\chi$ is an open map. We have shown that $\chi$ is a topological group isomorphism.

If $h$ is a continuous homomorphism $h: G \rightarrow H$ we show that the following diagram is commutative:

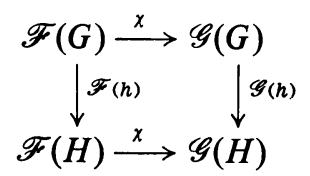

First, for each $\mu \in M_{G}\left(G_{1}, \ldots, G_{r}\right)$ and $d \in D$, it is clear that $\chi_{h \circ \mu}(d)=h\left(\chi_{\mu}(d)\right)$. Since $D$ is dense and $\chi_{h \circ \mu}$ and $h \circ \chi_{\mu}$ are continuous, it follows that $\chi_{h \circ \mu}=h \circ \chi_{\mu}$. Hence $\chi^{F}(h)(\mu)=\chi_{h \circ \mu}=h \circ \chi_{\mu}=\mathscr{G}(h) \chi_{\mu}$. Thus $\chi_{F}(h)=\mathscr{G}(h) \chi$. This completes the proof.

COROLLARY 1. If $M_{T}\left(G_{1}, \ldots, G_{r}\right)$ is locally compact the property of equivalence of r-linear maps and linear maps characterizes $\bigotimes_{i=1}^{r} G_{i}$ up to natural topological group isomorphisms. In particular, if all the $G_{i}$ are discrete then $\bigotimes_{i=1}^{r} G_{i}$ is the usual tensor product for abstract abelian groups.

Corollary 2. If $G, G_{1}, G_{2}$ and $M_{T}\left(G_{1}, G_{2}\right)$ are locally compact then Hom $\left(G_{1} \otimes G_{2}, G\right)$ is isomorphic with $\operatorname{Hom}\left(G_{1}\right.$, Hom $\left.\left(G_{2}, G\right)\right)$.

This follows directly from Theorems 4.7 and 4.2.

THeOREM 4.8. Let $G$ and $G_{1}, \ldots, G_{r}$ be abelian topological groups, and let $H$ and $H_{1}, \ldots, H_{r}$ be subgroups of the respective $G$ 's. Denote the natural epimorphisms $G \rightarrow G / H$ by $\pi$, and $\prod_{i=1}^{r} G_{\imath} \rightarrow \prod_{i=1}^{r} G_{i} / H_{i}$ by $f$. Suppose $\mu \in M_{G}\left(G_{1}, \ldots, G_{r}\right)$, and $\mu\left(G_{1} \times \cdots \times H_{i} \times \cdots \times G_{r}\right) \subset H$ for $i=1, \ldots, r$. Then there exists a unique $\nu \in M_{G / H}\left(G_{1} / H_{1}, \ldots, G_{r} / H_{r}\right)$ with the property that $\pi \mu=\nu f$. 
Proof. It is easy to see that for $\left(x_{1}, \ldots, x_{r}\right)$ and $\left(y_{1}, \ldots, y_{r}\right) \in \prod_{i=1}^{r} G_{i}$,

$$
\mu\left(x_{1}, \ldots, x_{r}\right)-\mu\left(y_{1}, \ldots, y_{r}\right)=\sum_{i=1}^{r} \mu\left(y_{1}, \ldots, y_{i-1}, x_{i}-y_{i}, x_{i+1}, \ldots, x_{r}\right) .
$$

Hence if $x_{i}+H_{i}=y_{i}+H_{i}$ for $i=1, \ldots, r$, then

$$
\mu\left(y_{i}, \ldots, y_{i-1}, x_{i}-y_{i}, x_{i+1}, \ldots, x_{r}\right) \in H
$$

for each $i$, and therefore $\sum_{i=1}^{r} \mu\left(y_{1}, \ldots, y_{i-1}, x_{i}-y_{i}, x_{i+1}, \ldots, x_{r}\right) \in H$, that is, $\mu\left(x_{1}, \ldots, x_{r}\right)+H=\mu\left(y_{1}, \ldots, y_{r}\right)+H$. Thus the equation $\pi \mu=\nu f$ yields a well defined $\nu$. Because $\mu$ is $r$-linear, $\nu$ is also $r$-linear. Since $\pi$ and $\mu$ are continuous and $f$ is open, it follows that $\nu$ is continuous. Clearly $\nu$ is unique.

COROLlaRY. In particular, if $\mu\left(G_{1} \times \cdots \times H_{i} \times \cdots \times G_{r}\right)=(0)$ for all $i$ then there is a unique $\nu \in M_{G}\left(G_{1} / H_{1}, \ldots, G_{r} / H_{r}\right)$ such that $\nu f=\mu$.

Definition. Let $G_{1}, \ldots, G_{r}$ be in $\mathscr{L}$ and for each $i$ let $H_{i}$ be a closed subgroup of $G_{i}$. Denote by $\left[H_{1}, \ldots, H_{r}\right]$ the closure of the subgroup of $\bigotimes_{i=1}^{r} G_{i}$ generated by $\left\{\phi\left(x_{1}, \ldots, x_{r}\right): x_{i} \in H_{i}\right.$ for some $\left.i=1, \ldots, r\right\}$, where $\phi$ is the tensor map on $\prod_{i=1}^{r} G_{i}$.

THEOREM 4.9. If $G_{1}, \ldots, G_{r}$ are compactly generated then $\bigotimes_{i=1}^{r}\left(G_{i} / H_{i}\right)$ is isomorphic with $\bigotimes_{i=1}^{r} G_{i} /\left[H_{1}, \ldots, H_{r}\right]$.

Proof. Since $\phi\left(G_{1} \times \cdots \times H_{i} \times \cdots \times G_{r}\right) \subset\left[H_{1}, \ldots, H_{r}\right]$ for $i=1, \ldots, r$ and $\phi$ is a continuous $r$-linear map, Theorem 4.8 shows that there is a continuous $r$-linear map $\nu: \prod_{i=1}^{r} G_{i} / H_{i} \rightarrow \bigotimes_{i=1}^{r} G_{i} /\left[H_{1}, \ldots, H_{r}\right]$ with the property that $\pi \phi=\nu f$ where $f: \prod_{i=1}^{r} G_{i} \rightarrow \prod_{i=1}^{r} G_{i} / H_{i}$ and $\pi: \bigotimes_{i=1}^{r} G_{i} \rightarrow \bigotimes_{i=1}^{r} G_{i} /\left[H_{1}, \ldots, H_{r}\right]$ are the canonical epimorphisms. Let $\phi^{\prime}$ be the tensor map on $\prod_{i=1}^{r} G_{i} / H_{i}$. By Theorem 4.6 there exists a continuous linear map $\chi_{v}: \bigotimes_{i=1}^{r} G_{i} / H_{i} \rightarrow \bigotimes_{i=1}^{r} G_{i} /\left[H_{1}, \ldots, H_{r}\right]$ satisfying $\chi_{\nu} \phi^{\prime}=\nu$. Similarly, since $\phi^{\prime} f$ is continuous $r$-linear there is a continuous linear map $\xi: \bigotimes_{i=1}^{r} G_{i} \rightarrow \bigotimes_{i=1}^{r} G_{i} / H_{i}$ with the property that $\xi \phi=\phi^{\prime} f$ so that $\chi_{\nu} \xi \phi=\chi_{\nu} \phi^{\prime} f$ $=v f=\pi \phi$. Since $M_{T}\left(G_{1}, \ldots, G_{r}\right)$ is locally compact, by Theorem 4.1, it follows from Theorem 4.6 that $\chi_{v} \xi=\pi$. Because $\xi$ is continuous and $\pi$ is open, $\chi_{v}$ is open. Since $\pi$ is surjective, so is $\chi_{v}$. It remains to show that $\chi_{v}$ is a monomorphism.

Let $\sum_{j} \phi\left(x_{1}^{(j)}, \ldots, x_{r}^{(j)}\right) \in \bigotimes_{i=1}^{r} G_{i}$ where for each $j$ one of the $x_{i}^{(j)} \in H_{i}$ for some $i=1, \ldots, r$. Then

$$
\xi\left(\sum_{j} \phi\left(x_{1}^{(j)}, \ldots, x_{r}^{(j)}\right)\right)=\sum_{j} \xi \phi\left(x_{1}^{(j)}, \ldots, x_{r}^{(j)}\right)=\sum_{j} \phi^{\prime} f\left(x_{1}^{(j)}, \ldots, x_{r}^{(j)}\right)=\sum_{j} 0=0
$$

since for each $j, \phi^{\prime} f\left(x_{1}^{(j)}, \ldots, x_{r}^{(j)}\right)=\phi^{\prime}(\ldots, 0, \ldots)$ and $\phi^{\prime}$ is $r$-linear. Since $\xi$ is continuous it follows that $\xi\left[H_{1}, \ldots, H_{r}\right]=(0)$. Hence there is a continuous linear $\operatorname{map} \xi^{-}: \bigotimes_{i=1}^{r} G_{i} /\left[H_{1}, \ldots, H_{r}\right] \rightarrow \bigotimes_{i=1}^{r} G_{i} / H_{i}$ satisfying $\xi^{-} \pi=\xi$, so that $\xi^{-} \chi_{v} \xi \phi$ $=\xi^{-} \pi \phi=\xi \phi$. Hence $\xi^{-} \chi_{\nu} \xi=\xi$ by Theorem 4.6, and therefore $\xi^{-} \chi_{\nu}$ is the identity on $\xi\left(\bigotimes_{i=1}^{r} G_{i}\right)$. Now, $\xi\left(\bigotimes_{i=1}^{r} G_{i}\right) \supset \xi \phi\left(\prod_{i=1}^{r} G_{i}\right)=\phi^{\prime} f\left(\prod_{i=1}^{r} G_{i}\right)=\phi^{\prime}\left(\prod_{i=1}^{r} G_{i} / H_{i}\right)$ 
since $f$ is surjective. Consequently $\xi\left(\bigotimes_{i=1}^{r} G_{i}\right)$ contains the subgroup $D^{\prime}$ of $\bigotimes_{i=1}^{r} G_{i} / H_{i}$ generated by $\phi^{\prime}\left(\prod_{i=1}^{r} G_{i} / H_{i}\right)$. Since $G_{i} / H_{i}$ is compactly generated, by Theorem 2.6, $M_{T}\left(G_{1} / H_{1}, \ldots, G_{r} / H_{r}\right)$ is locally compact, by Theorem 4.1 , and hence $D^{\prime}$ is dense, by Theorem 4.5. Thus $\xi\left(\bigotimes_{i=1}^{r} G_{i}\right)$ is dense. Since $\xi^{-} \chi_{v}$ is the identity on a dense subgroup it equals the identity everywhere, and therefore $\chi_{v}$ is a monomorphism. This completes the proof.

COROLlaRY. Under the hypothesis of Theorem 4.9, if the $H_{i}$ are all open then $\left[H_{1}, \ldots, H_{r}\right]$ is open.

Proof. If the $H_{i}$ are open then each $G_{i} / H_{i}$ is discrete and hence $\bigotimes_{i=1}^{r} G_{i} / H_{i}$ is discrete. By Theorem 4.9, $\bigotimes_{i=1}^{r} G_{i} /\left[H_{1}, \ldots, H_{r}\right] \cong \bigotimes_{i=1}^{r} G_{i} / H_{i}$. Therefore, $\left[H_{1}, \ldots, H_{r}\right]$ is open.

Proposition 4.1. Let $G$ be compactly generated, $D$ discrete and $R$ the additive group of real numbers. Then $\operatorname{Hom}(G, R) \cong R^{n+m}$ and $\operatorname{Hom}(G, D) \cong D^{m}$ $\oplus \operatorname{Hom}(C, D)$ where $G=R^{n} \oplus Z^{m} \oplus C$ is the canonical representation of $G$ in terms of its maximum compact subgroup $C$.

Proof. By Corollary 2 of Theorem 4.2, $\operatorname{Hom}(G, R) \cong \operatorname{Hom}\left(R^{\wedge}, G^{\wedge}\right)$. However, $G^{\wedge} \cong R^{n} \oplus T^{m} \oplus C^{\wedge}$ and $R^{\wedge} \cong R$. A previous remark yields $\operatorname{Hom}(G, R)$ $\cong \operatorname{Hom}(R, R)^{n} \oplus \operatorname{Hom}(R, T)^{m} \oplus \operatorname{Hom}\left(R, C^{\wedge}\right)$. It is clear that $\operatorname{Hom}(R, R) \cong R$. Since $R$ is connected and $C^{\wedge}$ is discrete, $\operatorname{Hom}\left(R, C^{\wedge}\right)=(0)$. Thus $\operatorname{Hom}(G, R)$ $\cong R^{n} \oplus\left(R^{\wedge}\right)^{m} \cong R^{n+m}$.

Similarly, $\operatorname{Hom}(G, D) \cong \operatorname{Hom}\left(D^{\wedge}, G^{\wedge}\right)$, which is isomorphic to $\operatorname{Hom}\left(D^{\wedge}, R\right)^{n}$ $\oplus \operatorname{Hom}\left(D^{\wedge}, T\right)^{m} \oplus \operatorname{Hom}\left(D^{\wedge}, C^{\wedge}\right)$. It is clear that $\operatorname{Hom}\left(D^{\wedge}, R\right)=(0)$, $\operatorname{Hom}\left(D^{\wedge}, T\right) \cong D$ and $\operatorname{Hom}\left(D^{\wedge}, C^{\wedge}\right) \cong \operatorname{Hom}(C, D)$. Thus $\operatorname{Hom}(G, D) \cong D^{m}$ $\oplus \operatorname{Hom}(C, D)$. This completes the proof.

THEOREM 4.10. If $G_{1}$ is a closed subgroup of a compactly generated group $G_{2}$ and $H$ has no small subgroups then the continuous restriction homomorphism

is open.

$$
f: \operatorname{Hom}\left(G_{2}, H\right) \rightarrow \operatorname{Hom}\left(G_{1}, H\right)
$$

Proof. Since $H$ has no small subgroups, $H \cong R^{n} \oplus T^{m} \oplus D$ where $D$ is a discrete group. As we have observed before, $\operatorname{Hom}\left(G_{i}, H\right)$ is naturally isomorphic with $\operatorname{Hom}\left(G_{i}, R\right)^{n} \oplus \operatorname{Hom}\left(G_{i}, T\right)^{m} \oplus \operatorname{Hom}(G, D)$. An easy direct sum argument shows that it suffices to prove that the following partial maps are open

$$
\begin{aligned}
& f_{1}: \operatorname{Hom}\left(G_{2}, R\right) \rightarrow \operatorname{Hom}\left(G_{1}, R\right), \\
& f_{2}: \operatorname{Hom}\left(G_{2}, T\right) \rightarrow \operatorname{Hom}\left(G_{1}, T\right), \\
& f_{3}: \operatorname{Hom}\left(G_{2}, D\right) \rightarrow \operatorname{Hom}\left(G_{1}, D\right) .
\end{aligned}
$$

(1) Since $R$ is injective, $f_{1}$ is an epimorphism. Both $\operatorname{Hom}\left(G_{1}, R\right)$ and $\operatorname{Hom}\left(G_{2}, R\right)$ are locally compact, by a corollary to Theorem 4.1. In fact, Proposition 4.1 shows 
that $\operatorname{Hom}\left(G_{2}, R\right)$ is a vector group. The open mapping theorem guarantees that $f_{1}$ is open.

(2) It is easy to see that $f_{2}=i^{\wedge}$ where $i: G_{1} \rightarrow G_{2}$ is the inclusion of $G_{1}$ into $G_{2}$. Hence $f_{2}$ is a proper epimorphism.

(3) $\operatorname{Hom}\left(G_{1}, D\right) \cong D^{m} \oplus \operatorname{Hom}(C, D)$, by Proposition 4.1. But the latter is isomorphic to $D^{m} \oplus M_{T}\left(C, D^{\wedge}\right)$. By Theorem 4.1, $M_{T}\left(C, D^{\wedge}\right)$ is discrete, because $C$ and $D^{\wedge}$ are compact. Thus $\operatorname{Hom}\left(G_{1}, D\right)$ is a finite direct product of discrete groups. Since $\operatorname{Hom}\left(G_{1}, D\right)$ is discrete, $f_{3}$ is an open map.

V. Tensor products of homomorphisms, exactness, and structural properties. Let $G_{1}, \ldots, G_{r}$ and $H_{1}, \ldots, H_{r}$ be in $\mathscr{L}$ and assume $M_{T}\left(G_{1}, \ldots, G_{r}\right)$ and $M_{T}\left(H_{1}, \ldots, H_{r}\right)$ are locally compact. Denote the respective tensor maps by $\phi: \prod_{i=1}^{r} G_{i} \rightarrow \bigotimes_{i=1}^{r} G_{i}$ and $\phi^{\prime}: \prod_{i=1}^{r} H_{i} \rightarrow \bigotimes_{i=1}^{r} H_{i}$. If for each $i=1, \ldots, r$ we are given a continuous homomorphism $f_{i}: G_{i} \rightarrow H_{i}$, define the map

$$
\left[f_{1}, \ldots, f_{r}\right]: \prod_{i=1}^{r} G_{i} \rightarrow \prod_{i=1}^{r} H_{i}
$$

by $\left[f_{1}, \ldots, f_{r}\right]\left(x_{1}, \ldots, x_{r}\right)=\left(f_{1}\left(x_{1}\right), \ldots, f_{r}\left(x_{r}\right)\right)$. It is clear that $\phi^{\prime}\left[f_{1}, \ldots, f_{r}\right]$ is a continuous $r$-linear map $\prod_{i=1}^{r} G_{i} \rightarrow \bigotimes_{i=1}^{r} H_{i}$. Since $M_{T}\left(H_{1}, \ldots, H_{r}\right)$ is in $\mathscr{L}$, so is $\bigotimes_{i=1}^{r} H_{i}$. Hence since $M_{T}\left(G_{1}, \ldots, G_{r}\right)$ is in $\mathscr{L}$ there exists, by Theorem 4.6, a unique continuous linear map denoted by $f_{1} \otimes \cdots \otimes f_{r}: \bigotimes_{i=1}^{r} G_{i} \rightarrow \bigotimes_{i=1}^{r} H_{i}$ satisfying $\left(f_{1} \otimes \cdots \otimes f_{r}\right) \cdot \phi=\phi^{\prime}\left[f_{1}, \ldots, f_{r}\right]$.

If, for $i=1, \ldots, r, g_{i}: G_{i} \rightarrow H_{i}$ is another family of continuous homomorphisms then for each $i, f_{1} \otimes \cdots \otimes f_{i}+g_{i} \otimes \cdots \otimes f_{r}=f_{1} \otimes \cdots \otimes f_{i} \otimes \cdots \otimes f_{r}+f_{1}$ $\otimes \cdots \otimes g_{1} \otimes \cdots \otimes f_{r}$. These equations are derived by observing that the functions on either side agree on $\phi\left(\prod_{i=1}^{r} G_{i}\right)$ and hence, by Theorem 4.6, on $\bigotimes_{i=1}^{r} G_{i}$. In particular, if $f_{i}=0$ for some $i$ then $f_{1} \otimes \cdots \otimes f_{r}=0$. It is clear that if id $_{G_{t}}$ is the identity map on $G_{i}$ then $\mathrm{id}_{G_{1}} \otimes \cdots \otimes \mathrm{id}_{G_{r}}=\mathrm{id} \otimes_{i=1}^{r} G_{i}$. Given continuous homomorphisms

$$
G_{i} \stackrel{f_{i}}{\longrightarrow} H_{i} \stackrel{g_{i}}{\longrightarrow} L_{i}
$$

where $i=1, \ldots, r$, a similar application of Theorem 4.6 shows that $g_{1} f_{1} \otimes \ldots$ $\otimes g_{r} f_{r}=\left(g_{1} \otimes \cdots \otimes g_{r}\right) \cdot\left(f_{1} \otimes \cdots \otimes f_{r}\right)$. If $f_{i}: G_{i} \rightarrow H_{i}$ is an isomorphism then $f_{1} \otimes \cdots \otimes f_{r}$ is an isomorphism and $\left(f_{1} \otimes \cdots \otimes f_{r}\right)^{-1}=f_{1}^{-1} \otimes \cdots \otimes f_{r}^{-1}$.

Dually, we define $\left[f_{1}, \ldots, f_{r}\right]^{\bullet}: M_{T}\left(H_{1}, \ldots, H_{r}\right) \rightarrow M_{T}\left(G_{1}, \ldots, G_{r}\right)$ by

$$
\left[f_{1}, \ldots, f_{r}\right]^{\bullet}(\mu)=\mu \cdot\left[f_{1}, \ldots, f_{r}\right]
$$

for $\mu \in M_{T}\left(H_{1}, \ldots, H_{r}\right)$. One sees easily that $\omega\left[f_{1}, \ldots, f_{r}\right]^{\bullet}=\left(f_{1} \otimes \cdots \otimes f_{r}\right)^{\wedge} \omega^{\prime}$ where $\omega$ denotes the natural isomorphism $M_{T}\left(G_{1}, \ldots, G_{r}\right) \rightarrow M_{T}\left(G_{1}, \ldots, G_{r}\right)^{\wedge \wedge}$ and $\omega^{\prime}$ the natural isomorphism $M_{T}\left(H_{1}, \ldots, H_{r}\right) \rightarrow M_{T}\left(H_{1}, \ldots, H_{r}\right)^{\wedge}$. Hence $\left[f_{1}, \ldots, f_{r}\right]^{\bullet}$ is the continuous homomorphism dual to $f_{1} \otimes \cdots \otimes f_{r}$. Whenever it is clear which maps $f_{1}, \ldots, f_{r}$ are being considered we will abbreviate $\left[f_{1}, \ldots, f_{r}\right]^{\bullet}$ by ${ }^{\bullet}$, and the image of a subset $S$ of $M_{T}\left(H_{1}, \ldots, H_{r}\right)$ in $M_{T}\left(G_{1}, \ldots, G_{r}\right)$ by $S^{\bullet}$. 
THEOREM 5.1. Let $M_{T}\left(G_{1}, \ldots, G_{r}\right)$ and $M_{T}\left(H_{1}, \ldots, H_{r}\right)$ be locally compact. If $f_{1}, \ldots, f_{r}$ are proper epimorphisms then $f_{1} \otimes \cdots \otimes f_{r}$ is a proper epimorphism.

Proof. Clearly $\left[f_{1}, \ldots, f_{r}\right]$ is an epimorphism. Consequently,

$$
\left(f_{1} \otimes \cdots \otimes f_{r}\right) \phi\left(\prod_{i=1}^{r} G_{i}\right)=\phi^{\prime}\left[f_{1}, \ldots, f_{r}\right]\left(\prod_{i=1}^{r} G_{i}\right)=\phi^{\prime}\left(\prod_{i=1}^{r} H_{i}\right) .
$$

It follows by linearity that $f_{1} \otimes \cdots \otimes f_{r}(D)=D^{\prime}$ where $D$ and $D^{\prime}$ are the subgroups of $\bigotimes_{i=1}^{r} G_{i}$ and $\bigotimes_{i=1}^{r} H_{i}$ generated by $\phi\left(\prod_{i=1}^{r} G_{i}\right)$ and $\phi^{\prime}\left(\prod_{i=1}^{r} H_{i}\right)$ respectively. Hence $f_{1} \otimes \cdots \otimes f_{r}\left(\bigotimes_{i=1}^{r} G_{i}\right)$ is dense in $\bigotimes_{i=1}^{r} H_{i}$. This implies that ${ }^{\bullet}$ is a (continuous) monomorphism. We show that ${ }^{\bullet}$ is proper. Let $W(F, \Gamma)$ be an open neighborhood of 0 in $M_{T}\left(H_{1}, \ldots, H_{r}\right)$ where $F$ is compact. Clearly $F$ is contained in $\prod_{i=1}^{r} F_{H_{i}}$, a compact box. Hence $W(F, \Gamma)^{\bullet} \supset W\left(\prod_{i=1}^{r} F_{H_{i}}, \Gamma\right)^{\bullet}$. It is sufficient to show that $W\left(\prod_{i=1}^{r} F_{H_{i}}, \Gamma\right)^{\bullet}$ contains the intersection of $M_{T}\left(H_{1}, \ldots, H_{r}\right)^{\bullet}$ with an open neighborhood of 0 in $M_{T}\left(G_{1}, \ldots, G_{r}\right)$. Thus we may assume $F=\prod_{i=1}^{r} F_{H_{i}}$. Let $U_{i}$ be a compact neighborhood of 0 in $G_{i}$. Since $f_{i}$ is a proper epimorphism, $\left\{f_{i}\left(x_{i}+U_{i}^{\circ}\right): x_{i} \in G_{i}\right\}$ is an open covering of $H_{i}$ and hence of $F_{H_{i}}$. By compactness $F_{H_{i}} \subset \bigcup_{j=1}^{n_{i}} f_{i}\left(x_{i}^{(j)}+U_{i}^{\circ}\right)$ where $x_{i}^{(j)} \in G_{i}$. Let

$$
F_{G_{i}}=\bigcup_{j=1}^{n_{i}} x_{i}^{(j)}+U_{i}
$$

It is clear that $F_{G_{i}}$ is compact and $f_{i}\left(F_{G_{i}}\right) \supset F_{H_{i}}$. Evidently, $W\left(\prod_{i=1}^{r} F_{H_{i}}, \Gamma\right)^{\bullet}$ $\supset M_{T}\left(H_{1}, \ldots, H_{r}\right)^{\bullet} \cap W\left(\prod_{i=1}^{r} F_{G_{i}}, \Gamma\right)$. Thus $\bullet$ is a proper monomorphism and hence $f_{1} \otimes \cdots \otimes f_{r}$ is a proper epimorphism, by Theorem 2.1. This completes the proof.

Henceforth we restrict ourselves to the case $r=2$ and slightly change the notation.

Proposition 5.1. If $g: G_{1} \rightarrow G_{2}$ and $h: H_{1} \rightarrow H_{2}$ are proper epimorphisms where $G_{i}$ and $H_{i} \in \mathscr{L}$ for $i=1,2$, then $M_{T}\left(G_{2}, H_{2}\right)^{\bullet}$ is the set of $\nu \in M_{T}\left(G_{1}, H_{1}\right)$ satisfying $\nu\left(G_{1} \times \operatorname{Ker} h\right)=(0)=\nu\left(\operatorname{Ker} g \times H_{1}\right)$.

Proof. Suppose $\nu \in M_{T}\left(G_{2}, H_{2}\right)^{\bullet}$. If $x \in \operatorname{Ker} g$, then $\nu(x, y)=\mu(0, h(y))=(0)$, since $\mu$ is bilinear. Hence $\nu\left(\operatorname{Ker} g \times H_{1}\right)=(0)$. Similarly, $\nu\left(G_{1} \times \operatorname{Ker} h\right)=(0)$. On the other hand, if $\nu \in M_{T}\left(G_{1}, H_{1}\right)$ and $\nu\left(G_{1} \times \operatorname{Ker} h\right)=(0)=\nu\left(\operatorname{Ker} g \times H_{1}\right)$, then by Theorem 4.8, $\nu$ induces a map $\mu$ in $M_{T}\left(G_{1} / \operatorname{Ker} g, H_{1} / \operatorname{Ker} h\right)$ with the property that $\mu f=\nu$, where $f$ is the map from $G_{1} \times H_{1} \rightarrow\left(G_{1} / \operatorname{Ker} g\right) \times\left(H_{1} / \operatorname{Ker} h\right)$, defined by $f(x, y)=(x+\operatorname{Ker} g, y+\operatorname{Ker} h)$. Since $G_{2} \cong G_{1} / \operatorname{Ker} g$, and $H_{2} \cong H_{1} / \operatorname{Ker} h$, after identification via these isomorphisms, $f=[g, h]$ and therefore, $\nu=\mu[g, h]$. This completes the proof.

THEOREM 5.2. Let

$$
0 \longrightarrow G_{1} \stackrel{g_{1}}{\longrightarrow} G_{2} \stackrel{g_{2}}{\longrightarrow} G_{3} \longrightarrow 0
$$


be a proper exact sequence with $G_{1}$ and $G_{3}$, or equivalently $G_{2}$, compactly generated. Let $H$ be compactly generated. Then

$$
G_{1} \otimes H \stackrel{g_{1} \otimes \mathrm{Id}_{H}}{\longrightarrow} G_{2} \otimes H \stackrel{g_{2} \otimes \mathrm{Id}_{H}}{\longrightarrow} G_{3} \otimes H \longrightarrow(0)
$$

is a proper exact sequence.

Proof. By Theorem 2.1, it is sufficient to show that

$$
0 \longrightarrow M_{T}\left(G_{3}, H\right) \stackrel{\bullet_{2}}{\longrightarrow} M_{T}\left(G_{2}, H\right) \stackrel{\bullet_{1}}{\longrightarrow} M_{T}\left(G_{1}, H\right)
$$

is proper exact. By Theorem $5.1, \bullet_{2}$ is a proper monomorphism. Since Ker ${ }^{\bullet_{1}}$ is the set of all $\mu \in M_{T}\left(G_{2}, H\right)$ that annihilate $g_{1}\left(G_{1}\right) \times H=\operatorname{Ker} g_{2} \times H$, it follows from Proposition 5.1 that $\operatorname{Ker} \bullet_{1}=M_{T}\left(G_{3}, H\right)^{\bullet_{2}}$. Because of the isomorphism of functors established in $\S \mathrm{IV}$, it follows from Theorem 4.10 that ${ }^{\bullet}{ }_{1}$ is proper. This completes the proof.

COROLLARY. Let $G$ be compactly generated and $Z_{n}$ be the cyclic group of order $n$. Then $Z_{n} \otimes G \cong G / n G$.

Proof. Consider the proper exact sequence $0 \rightarrow n Z \rightarrow Z \rightarrow Z_{n} \rightarrow 0$. By Theorem $5.2, n Z \otimes G \rightarrow Z \otimes G \rightarrow Z_{n} \otimes G \rightarrow 0$ is proper exact. Hence $Z_{n} \otimes G \cong Z$ $\otimes G$ /image of $n Z \otimes G$. However as we noted earlier this may be identified with $G / n G$. Note that $n G$ is a closed subgroup of $G$ since $G$ is compactly generated. This completes the proof.

Now let $\mathscr{C}$ and $\mathscr{N}$, respectively, denote the categories of groups in $\mathscr{L}$ that are compactly generated and have no small subgroups. We define four families of functors of $G$. These are defined only on the categories and only for those $H$ 's indicated below. The mappings are those already used several times.

(1) $* \otimes H: \mathscr{C} \rightarrow \mathscr{L}$, where $H$ is in $\mathscr{C}$. This is a covariant additive functor.

(2) $M_{T}(*, H): \mathscr{C} \rightarrow \mathscr{L}$, where $H$ is in $\mathscr{C}$. This is a contravariant additive functor.

(3) $\operatorname{Hom}(*, H): \mathscr{C} \rightarrow \mathscr{L}$, where $H$ is in $\mathscr{N}$. This is a contravariant additive functor.

(4) Hom $(H, *): \mathscr{N} \rightarrow \mathscr{L}$, where $H$ is in $\mathscr{C}$. This is a covariant additive functor.

Definition. A covariant additive functor $\mathscr{F}$ is called proper terminally exact if for all proper exact sequences $0 \rightarrow G_{1} \rightarrow G_{2} \rightarrow G_{3} \rightarrow 0$ in the domain of $\mathscr{F}$, $\mathscr{F}\left(G_{1}\right) \rightarrow \mathscr{F}\left(G_{2}\right) \rightarrow \mathscr{F}\left(G_{3}\right) \rightarrow 0$ is a proper exact sequence, and is called proper initially exact if $0 \rightarrow \mathscr{F}\left(G_{1}\right) \rightarrow \mathscr{F}\left(G_{2}\right) \rightarrow \mathscr{F}\left(G_{3}\right)$ is a proper exact sequence.

Similarly a contravariant additive functor $\mathscr{F}$ is called proper terminally exact if for all proper exact sequences $0 \rightarrow G_{1} \rightarrow G_{2} \rightarrow G_{3} \rightarrow 0$ in the domain of $\mathscr{F}$, $\mathscr{F}\left(G_{3}\right) \rightarrow \mathscr{F}\left(G_{2}\right) \rightarrow \mathscr{F}\left(G_{1}\right) \rightarrow 0$ is a proper exact sequence, and is called proper initially exact if $0 \rightarrow \mathscr{F}\left(G_{3}\right) \rightarrow \mathscr{F}\left(G_{2}\right) \rightarrow \mathscr{F}\left(G_{1}\right)$ is a proper exact sequence. These definitions are continuous analogues of those given in [6].

REMARK. Throughout $\S \mathrm{V}$, we will utilize fully the fact that all notions and theorems dualize; in particular, that $\mathscr{C}$ and $\mathscr{N}$ and certain of their subclasses are 
dual categories, that the dual of a short proper exact sequence is a short proper exact sequence, and that for $G$ and $H$ in $\mathscr{C},(G \otimes H)^{\wedge}, M_{T}(G, H)$, Hom $\left(G, H^{\wedge}\right)$ and $\operatorname{Hom}\left(H, G^{\wedge}\right)$ are isomorphic functors. Thus one proof replaces four.

In proving Theorem 5.2 , we have actually proven

THEOREM 5.3. The functor $* \otimes H$ is a proper terminally exact functor, and $M_{T}(*, H)$, Hom $(*, H)$, and $\operatorname{Hom}(H, *)$ are proper initially exact functors.

Corollary. Let $G_{1}$ and $G_{2}$ be in $\mathscr{C}$ and $H$ in $\mathscr{N}$. If $g: G_{1} \rightarrow G_{2}$ is a proper homomorphism then the induced map $g^{*}: \operatorname{Hom}\left(G_{2}, H\right) \rightarrow \operatorname{Hom}\left(G_{1}, H\right)$ obtained by composition is a proper homomorphism.

Let $G_{1}, G_{2}$ and $H$ be in $\mathscr{C}$. If $g: G_{1} \rightarrow G_{2}$ is a proper homomorphism then the induced maps $G_{1} \otimes H \rightarrow G_{2} \otimes H$ and $M_{T}\left(G_{2}, H\right) \rightarrow M_{T}\left(G_{1}, H\right)$ are proper homomorphisms.

Let $G_{1}$ and $G_{2}$ be in $\mathscr{N}$ and $H$ in $\mathscr{C}$. If $g: G_{1} \rightarrow G_{2}$ is a proper homomorphism then the induced map $\operatorname{Hom}\left(H, G_{1}\right) \rightarrow \operatorname{Hom}\left(H, G_{2}\right)$ is a proper homomorphism.

The above Corollary generalizes Theorem 4.10.

Proof. Since $g\left(G_{1}\right)$ is a closed subgroup of $G_{2}$, we can write $g=i \pi$ where $i: g\left(G_{1}\right) \rightarrow G_{2}$ and $\pi: G_{1} \rightarrow g\left(G_{1}\right)$ are proper homomorphisms; $i$ is injective and $\pi$ surjective. Hence $g^{*}=\pi^{*} i^{*}$. It follows from Theorem 4.10 that $i^{*}$ is an open map. Thus, to show that $g^{*}$ is proper, it is sufficient to show that $\pi^{*}$ is proper, that is, we are reduced to proving the theorem in the case where $g$ is surjective. In this case, consider the proper exact sequence $0 \rightarrow \operatorname{Ker} g \rightarrow G_{1} \rightarrow G_{2} \rightarrow 0$. It follows from Theorem 5.3 that

$$
0 \longrightarrow \operatorname{Hom}\left(G_{2}, H\right) \stackrel{g^{\bullet}}{\longrightarrow} \operatorname{Hom}\left(G_{1}, H\right) \longrightarrow \operatorname{Hom}(\operatorname{Ker} g, H)
$$

is a proper exact sequence. In particular, $g^{*}$ is a proper homomorphism. The proof is completed by dualization.

Definition. A covariant or contravariant additive functor $\mathscr{F}$ is called proper exact if it is both proper terminally exact and proper initially exact (see [6]).

THEOREM 5.4. $* \otimes H, M_{T}(*, H)$ and $\operatorname{Hom}(H, *)$ are proper exact functors if and only if the maximum compact subgroup of $H$ is $(0)$. Hom $(*, H)$ is a proper exact functor if and only if $H$ is connected.

Proof. Hom $(*, H)$ is proper exact if and only if $H$ is injective for the class of compactly generated groups. As we have already proven, the injectives for $\mathscr{L}$ are the same as those for compactly generated groups, namely, $R^{n} \oplus T^{\sigma}$, direct sums of vector groups and tori. $H$ is a group without small subgroups; hence, $\sigma$ is finite. So $\operatorname{Hom}(*, H)$ is a proper exact functor if and only if $H=R^{n} \oplus T^{m}$, i.e., if and only if $H$ is a connected group without small subgroups. Consequently, because of the isomorphism of various functors, and Theorems 2.1 and 2.5 Corollary 2 , the result follows. 
COROLlaRY. We remark that $\operatorname{Hom}(*, H)$ is proper exact if and only if it is proper exact relative to the sequence $0 \rightarrow Z \rightarrow R \rightarrow T \rightarrow 0$, for, as we saw earlier, a group which is injective for this sequence must be connected, and hence is injective for all sequences, by Theorem 5.4. Since the sequence $0 \rightarrow Z \rightarrow R \rightarrow T \rightarrow 0$ is self dual, the same statements are true for $* \otimes H, M_{T}(*, H)$ and $\operatorname{Hom}(H, *)$.

Definition. A functor preserving proper exactness for a class of extensions is called a proper exact functor relative to that class.

THEOREM 5.5. Relative to sequences $0 \rightarrow G_{1} \rightarrow G_{2} \rightarrow G_{3} \rightarrow 0$, where $G_{1}$ is open in $G_{2}, * \otimes H$ and $M_{T}(*, H)$ are proper exact if and only if $H$ is torsion free. Hom $(*, H)$ is proper exact if and only if $H$ is divisible. Relative to sequences $0 \rightarrow G_{1} \rightarrow G_{2} \rightarrow G_{3} \rightarrow 0$, where $G_{1}$ is compact, $\operatorname{Hom}(H, *)$ is proper exact if and only if $H$ is torsion free.

Proof. By dualization, it is sufficient to prove that $\operatorname{Hom}(*, H)$ is proper exact if and only if $H$ is divisible. If $\operatorname{Hom}(*, H)$ is proper exact relative to the sequences $0 \rightarrow n Z \rightarrow Z \rightarrow Z_{n} \rightarrow 0$ then evidently $H$ is divisible. The converse follows from the fact that $G_{1}$ is open in $G_{2}$.

THEOREM 5.6. Relative to sequences $0 \rightarrow G_{1} \rightarrow G_{2} \rightarrow G_{3} \rightarrow 0$ with each $G_{i}$ compact, $* \otimes H$ and $M_{T}(*, H)$ are proper exact if and only if the maximum compact subgroup of $H$ is connected. Hom $(*, H)$ is proper exact if and only if $H / H_{0}$ is torsion free. Relative to sequences $0 \rightarrow G_{1} \rightarrow G_{2} \rightarrow G_{3} \rightarrow 0$, of discrete groups, $\operatorname{Hom}(H, *)$ is proper exact if and only if the maximum compact subgroup of $H$ is connected.

Proof. If $H$ is connected, $\operatorname{Hom}(*, H)$ is proper exact, by Theorem 5.4. Thus we are led to the case of $H=D$, a discrete group. $D$ is torsion free; for suppose $y \in D$, and the order of $y=n$. Consider the sequence $0 \rightarrow Z_{n} \rightarrow T \rightarrow T \rightarrow 0$. The natural map of $Z_{n}$ into $D$ cannot extend to $T$ since $T$ is connected. Conversely, if $D$ is a torsion free discrete group, and $\xi: G_{1} \rightarrow D$ is a continuous homomorphism, then $\xi\left(G_{1}\right)$ is compact and hence finite, since $D$ is discrete. Because $D$ is torsion free, $\xi$ is trivial and extends trivially. Dualizing, Theorem 5.6 is completely proven.

THEOREM 5.7. Relative to sequences $0 \rightarrow G_{1} \rightarrow G_{2} \rightarrow G_{3} \rightarrow 0$, where $G_{2}$ is connected and $G_{1}$ is a pure subgroup of $G_{2}, * \otimes H$ and $M_{T}(*, H)$ are proper exact functors for all $H$ in $\mathscr{C}$. Hom $(*, H)$ is a proper exact functor for all $H$ in $\mathscr{N}$. Relative to sequences $0 \rightarrow G_{1} \rightarrow G_{2} \rightarrow G_{3} \rightarrow 0$ where $G_{2}$ and $G_{3}$ are torsion free, $\operatorname{Hom}(H, *)$ is a proper exact functor for all $H$ in $\mathscr{C}$.

Proof. If $H$ is connected, $\operatorname{Hom}(*, H)$ is proper exact, by Theorem 5.4. Thus, we are led to the case $H=D$, a discrete group. Now, by Corollary 1 of Theorem 2.9, $G_{1}$ is connected. Hence any continuous homomorphism $G_{1} \rightarrow D$ is trivial and extends trivially. The proof is completed by dualization.

Next we give an explicit computation of $\operatorname{Hom}\left(G_{1}, G_{2}\right), G_{1} \otimes G_{2}$, and $M_{T}\left(G_{1}, G_{2}\right)$ in terms of known invariants of $G_{1}$ and $G_{2}$. 
THEOREM 5.8. Let $G$ be compactly generated and $H$ have no small subgroups. Let their canonical decompositions be $G=R^{n} \oplus Z^{m} \oplus C$ and $H=R^{k} \oplus T^{l} \oplus D$. Then $\operatorname{Hom}(G, H) \cong R^{(n+m) k} \oplus\left(G^{\wedge}\right)^{l} \oplus D^{m} \oplus \operatorname{Hom}(C, D)$.

Dually, if $G_{1}$ and $G_{2}$ are compactly generated groups whose decompositions for $i=1,2$ are $G_{i}=R^{n_{i}} \oplus Z^{m_{i}} \oplus C_{i}$, where $C_{i}$ is the maximal compact subgroup, then

$$
G_{1} \otimes G_{2} \cong R^{\left(n_{1}+m_{1}\right) n_{2}} \oplus G_{1}^{m_{2}} \oplus C_{2}^{m_{1}} \oplus C_{1} \otimes C_{2},
$$

and

$$
M_{T}\left(G_{1}, G_{2}\right) \cong R^{\left(n_{1}+m_{1}\right) n_{2}} \oplus\left(G_{1}^{\wedge}\right)^{m_{2}} \oplus\left(C_{2}^{\wedge}\right)^{m_{1}} \oplus M_{T}\left(C_{1}, C_{2}\right) .
$$

Proof. The first part follows directly from the additive properties of Hom, and Proposition 4.1. The second part now follows by dualization.

We use Theorem 5.8 to compute some specific examples and give the significance of tensor products of various groups. The first such result gives a sharpening of Theorem 4.4.

CoRollary 1. If $G_{1}$ and $G_{2}$ are in $\mathscr{C}$ then so is $G_{1} \otimes G_{2}$.

Proof. The result follows from Theorem 5.8, since $C_{1} \otimes C_{2}$ is compact by Theorem 4.4.

Corollary 2. If $G_{1}$ is connected and $G_{2}$ is compactly generated then

$$
G_{1} \otimes G_{2} \cong R^{n_{1} n_{2}} \oplus G_{1}^{m} \text {. }
$$

Proof. $G_{1}$ is connected if and only if $m_{1}=0$ and $C_{1}$ is connected. In this case $C_{2}^{m_{1}}=(0)$ and $\left(C_{1} \otimes C_{2}\right)^{\wedge} \cong \operatorname{Hom}\left(C_{1}, C_{2}^{\wedge}\right)=(0)$. Hence $C_{1} \otimes C_{2}=(0)$. Thus $G_{1} \otimes G_{2} \cong R^{n_{1} n_{2}} \oplus G_{1}^{m}$. From this we conclude several facts:

(1) If $G_{1}$ is connected and $G_{2}$ is compactly generated then $G_{1} \otimes G_{2}$ is connected. This is clear.

(2) If $G_{1}$ is connected and $G_{2}$ is compact then $G_{1} \otimes G_{2}=(0) . G_{2}$ is compact if and only if $n_{2}=m_{2}=0$. The result follows.

(3) If $G_{1}$ is connected and $G_{2}$ is compact then $M_{G}\left(G_{1}, G_{2}\right)=(0)$ for all locally compact commutative groups $G$.

For $G=T$, we see that $M_{T}\left(G_{1}, G_{2}\right) \cong\left(G_{1} \otimes G_{2}\right)^{\wedge}=(0)$. Now let $G$ be any locally compact group and $\xi$ a character of $G$. If $\mu \in M_{G}\left(G_{1}, G_{2}\right)$ then $\xi \mu \in M_{T}\left(G_{1}, G_{2}\right)$, so that $\xi \mu\left(x_{1}, x_{2}\right)=0$ for all characters $\xi$. Since the characters of $G$ separate points, $\mu=0$.

(4) If $G_{1}$ and $G_{2}$ are connected then $G_{1} \otimes G_{2} \cong R^{n_{1} n_{2}}$. The tensor product of connected groups of vector dimensions $n_{1}$ and $n_{2}$ is isomorphic to the vector group of dimension $n_{1} n_{2}$.

(5) If $G$ has no small subgroups then $T \otimes G^{\wedge}$ is (naturally $\cong$ with) the maximal toral subgroup of $G$.

We take $G_{1}=T$ and $G_{2}=G^{\wedge}$, a compactly generated group. Since $n_{1}=m_{1}=0$ and $C_{1}=T$, it follows that $T \otimes G^{\wedge}=T^{m_{2}}$, which is the maximal toral subgroup of $G$. 
COROLlaRY 3. If $G$ is a group without small subgroups then $\operatorname{Hom}(R, G)$, the group of one parameter subgroups of $G$, together with $e: \operatorname{Hom}(R, G) \rightarrow G$, the evaluation at 1 , is the simply connected covering group of $G_{0}$.

Proof. $G=R^{n} \oplus T^{m} \oplus D$. Since $\operatorname{Hom}(R, G) \cong M_{T}\left(R, G^{\wedge}\right)$, taking $n_{2}=1, m_{2}=0$ and $C_{2}=(0)$, we see that $\operatorname{Hom}(R, G) \cong R^{n+m}=R^{\mathrm{dim} G}$. Since uniform convergence on compact subsets implies pointwise convergence, the evaluation map $e$ is a continuous homomorphism. Because $\operatorname{Hom}(R, G)$ is a vector group, it is connected. This means that $e(\operatorname{Hom}(R, G))$ is contained in $G_{0}$. Since $G_{0}$ is connected, the corollary to Theorem 5.4 shows that $\operatorname{Hom}\left(*, G_{0}\right)$ is proper exact for the sequence $0 \rightarrow Z \rightarrow R \rightarrow T \rightarrow 0$. Hence each point of $G_{0}$ lies on a one parameter subgroup, and $e$ is surjective, as a map to $G_{0}$. Since both groups are compactly generated the Open Mapping Theorem shows that $e$ is proper. A comparison of the dimensions of $\operatorname{Hom}(R, G)$ and $G_{0}$ shows that $\operatorname{Ker} e$ is discrete, and so $e$ is a covering map. Since $\operatorname{Hom}(R, G)$ is a vector group the result follows.

Now we turn to $G \otimes H$ where $G$ and $H$ are compact.

Proposition 5.1. Let $G$ be in $\mathscr{C}$ and $H$ be compact. Then $G \otimes H \cong\left(G / G_{0}\right) \otimes H$.

Proof. Consider the proper exact sequence $0 \rightarrow G_{0} \rightarrow G \rightarrow G / G_{0} \rightarrow 0$. It follows that $G_{0} \otimes H \rightarrow G \otimes H \rightarrow\left(G / G_{0}\right) \otimes H \rightarrow 0$ is proper exact. However, $\left(G_{0} \otimes H\right)^{\wedge}$ $\cong \operatorname{Hom}\left(G_{0}, H^{\wedge}\right)$. Since $G_{0}$ is connected and $H^{\wedge}$ is discrete, $\operatorname{Hom}\left(G_{0}, H^{\wedge}\right)=(0)$, and therefore $G_{0} \otimes H=(0)$. This means that $0 \rightarrow G \otimes H \rightarrow\left(G / G_{0}\right) \otimes H \rightarrow 0$ is proper exact, and consequently $G \otimes H \cong\left(G / G_{0}\right) \otimes H$.

CoRollary. If $G$ and $H$ are compact then $G \otimes H \cong\left(G / G_{0}\right) \otimes\left(H / H_{0}\right)$.

Proof. By repeating the above argument we get $\left(G / G_{0}\right) \otimes H \cong\left(G / G_{0}\right) \otimes\left(H / H_{0}\right)$, and hence $G \otimes H \cong\left(G / G_{0}\right) \otimes\left(H / H_{0}\right)$, the tensor product of compact totally disconnected groups.

This suggests utilization of results of Braconnier [5], some of which, however, go back to Krull and others.

We denote by $I_{p}$ the additive group of the $p$-adic integers, by $\left(Z, \|_{p}\right)$ the subgroup of integers with the $p$-adic topology and by $Z\left(p^{n}\right)$ the cyclic group of order $p^{n}$. $I_{p}$ is a compact commutative totally disconnected group.

Now we give the definition of a $p$-group in [5], and summarize some of the basic properties we shall utilize. Let $G$ be in $\mathscr{L}$ and define $f: Z \times G \rightarrow G$ by $f(n, x)=n x$.

Definition. $G$ is a $p$-group if for each fixed $x \in G, f$ is continuous in $n$, where $Z$ takes the $p$-adic topology. If $G$ is discrete, this notion coincides with the usual one of a $p$-group in abstract abelian groups (see [3]). If $G$ is a $p$-group then $f$ extends to a continuous bilinear map, say $\mu: I_{p} \times G \rightarrow G$. $G$ is a $p$-group if and only if $G^{\wedge}$ is a $p$-group. $I_{p}$ is a $p$-group.

Definition. Let $G \in \mathscr{L} . G_{p}$ is the set of those $x$ 's in $G$ for which $f(*, x)$ is a continuous function of $n$, where $Z$ takes the $p$-adic topology. $G_{p}$ is called the $p$ primary component of $G$. 
The $G_{p}$ 's are disjoint $p$ subgroups of $G$. If $G$ is totally disconnected then $G_{p}$ is closed. If $G$ is compact and totally disconnected then $G \cong \prod_{p} G_{p}$, the direct product of the $G_{p}$ 's (product topology). This is called the primary decomposition of a compact totally disconnected group [5]. It is dual to the primary decomposition of a discrete torsion group [3].

Proposition 5.2. If $G$ is a compact p-group, then $I_{p} \otimes G \cong G$.

Proof. Let $\mu$ be the $G$-valued bilinear map on $I_{p} \times G$, derived from the fact that $G$ is a locally compact $p$-group. Let $\chi_{\mu}$ be the induced continuous homomorphism satisfying $\chi_{\mu} \phi=\mu . \chi_{\mu}$ is surjective, since $\chi_{\mu}\left(I_{p} \otimes G\right) \supset \chi_{\mu} \phi\left(I_{p} \times G\right)$ $=\mu\left(I_{p} \times G\right) \supset \mu\left(\left(Z, \|_{p}\right) \times G\right)$. But $\mu(1, g)=g$, so that $\mu\left(\left(Z, \|_{p}\right) \times G\right) \supset G$. Thus $\chi_{\mu}\left(I_{p} \otimes G\right)=G$. Since $I_{p}$ and $G$ are compact; so is $I_{p} \otimes G$. Thus the Open Mapping Theorem yields that $\chi_{\mu}$ is open. We consider $\widehat{\chi_{\mu}}: G^{\wedge} \rightarrow M_{T}\left(I_{p}, G\right)$, defined by $\widehat{\chi_{\mu}}(\xi)=\xi \circ \mu$ for $\xi \in G^{\wedge}$, and show that $\widehat{\chi_{\mu}}$ is surjective. Suppose $\nu \in M_{T}\left(I_{p}, G\right)$. We consider the restriction of $\nu$ to $\left(Z, \|_{p}\right) \times G$. Let $\xi(x)=\nu(1, x)$. Clearly $\xi$ is a character of $G$. Now $\xi \mu(n, x)=\xi(n, x)=n \xi(x)=n \nu(1, x)=\nu(n, x)$. So $\xi \mu=\nu$ on $\left(Z, \|_{p}\right) \times G$ which is dense in $I_{p} \times G$, because $\left(Z, \|_{p}\right)$ is dense in $I_{p}$. Since $\xi \mu$ and $\nu$ are continuous, they are equal. Thus $\widehat{\chi_{\mu}}$ is surjective. Since $G$ and $M_{T}\left(I_{p}, G\right)$ are locally compact, this implies that $\chi_{\mu}$ is injective and hence an isomorphism.

Proposition 5.3. If $G$ is any compact totally disconnected group then $I_{p} \otimes G \cong G_{p}$.

Proof. Let $G \cong \Pi_{q} G_{q}$ be the primary decomposition of $G$. $I_{p} \otimes G \cong \Pi_{q}\left(I_{p} \otimes G_{q}\right)$. Now $I_{p} \otimes G_{q}=(0)$ for $q \neq p$. In fact, $\left(I_{p} \otimes G_{q}\right)^{\wedge} \cong \operatorname{Hom}\left(I_{p}, G_{q}^{\wedge}\right)$ where $G_{q}^{\wedge}$ is a discrete $q$ group. If $\xi: I_{p} \rightarrow G_{q}$ is a continuous homomorphism we know [5] that either (1) $\xi\left(I_{p}\right) \cong I_{p}$, or (2) $\xi\left(I_{p}\right) \cong Z\left(p^{n}\right)$ depending on whether $\xi$ is injective or not. In any case, $I_{p}$ is torsion free, and $G_{q}^{\wedge}$ is a discrete $q$ group. Hence (1) is impossible. Similarly, since $Z\left(p^{n}\right)$ has all its elements of order $p^{k}$, with $p \neq q$, (2) is impossible. Thus $\operatorname{Hom}\left(I_{p}, G_{q}^{\wedge}\right)=(0)$, and therefore $I_{p} \otimes G_{q}=(0)$. Hence $I_{p} \otimes G \cong \prod_{q} I_{p} \otimes G_{q}$ $\cong I_{p} \otimes G_{p} \cong G_{p}$, by Proposition 5.2.

THEOREM 5.9. If $G$ is any compact group, then

(1) $I_{p} \otimes G \cong\left(G / G_{0}\right)_{p}$, the p-primary component of $G / G_{0}$.

(2) $I_{p} \otimes I_{q}=(0)$, for $q \neq p$, and $I_{p} \otimes I_{p} \cong I_{p}$.

(3) $G / G_{0} \cong \prod_{p}\left(I_{p} \otimes G\right)$.

Proof. $I_{p} \otimes G \cong I_{p} \otimes\left(G / G_{0}\right)$ by Proposition 5.1. By Proposition 5.3, $I_{p} \otimes\left(G / G_{0}\right)$ $\cong\left(G / G_{0}\right)_{p}$. Hence, $I_{p} \otimes G \cong\left(G / G_{0}\right)_{p}$. In particular, $I_{p} \otimes I_{q}=(0)$, for $q \neq p$, and $I_{p} \otimes I_{p} \cong I_{p}$. It follows that, for any compact group $G, G / G_{0} \cong \prod_{p} I_{p} \otimes G$. This completes the proof.

Corollary. Let $G$ be in $\mathscr{C}$. Then $G \otimes H=(0)$ for all compact groups $H$ if and only if $G$ is connected.

Proof. If $H$ is compact and $G$ is connected, then $G \otimes H=(0)$ by Corollary 3 of Theorem 5.8. Conversely, suppose $G \otimes H=(0)$ for all compact groups $H$. 
We have $G \cong R^{n} \oplus Z^{m} \oplus C$, and hence $G \otimes H \cong(R \otimes H)^{n} \oplus(Z \otimes H)^{m} \oplus C \otimes H$. As was just noted, $R \otimes H=(0)$. Also, $Z \otimes H \cong H$. Since $G \otimes H=(0)$ for all $H$, it follows that $m=0$. Because $R^{n}$ is connected and $G \otimes H \cong C \otimes H$, we may assume that $G$ is compact. By hypothesis, $I_{p} \otimes G=(0)$ for each prime $p$. Hence, by Theorem 5.9, $G / G_{0} \cong \prod_{p} I_{p} \otimes G=(0)$. Thus $G=G_{0}$.

THEOREM 5.10. (1) The tensor product of compact totally disconnected groups is a compact totally disconnected group whose p-primary component is $G_{p} \otimes H_{p}$.

(2) The tensor product of compact p-groups is a compact p-group.

(3) The tensor product of a compact p-group with a compact q-group is (0) for $p \neq q$.

Proof. Let $G$ and $H$ be arbitrary compact groups. Then $G \otimes H$ is compact by Theorem 4.4. It is totally disconnected if and only if $(G \otimes H)^{\wedge} \cong \operatorname{Hom}\left(G, H^{\wedge}\right)$ is a torsion group. Now if $f \in \operatorname{Hom}\left(G, H^{\wedge}\right)$ then $f(G)$ is a finite subgroup of $H^{\wedge}$, since $G$ is compact and $H^{\wedge}$ is discrete. Let $n(f)=$ order of $f(G)$. Then $n(f) f=0$. Thus $(G \otimes H)^{\wedge}$ is a torsion group, whence $G \otimes H$ is compact and totally disconnected.

Now suppose, in addition, that $G$ and $H$ are totally disconnected. Let $G=\prod_{p} G_{p}$ and $H=\prod_{q} H_{q}$ be the primary decompositions of $G$ and $H$, respectively. Then $G \otimes H \cong \prod_{p, q} G_{p} \otimes H_{q}$. Now if $p \neq q$ then

$$
G_{p} \otimes H_{q} \cong I_{p} \otimes G \otimes I_{q} \otimes H \cong I_{p} \otimes I_{q} \otimes G \otimes H \cong(0) \otimes G \otimes H=(0) .
$$

Hence, $G \otimes H \cong \prod_{p} G_{p} \otimes H_{p}$. Moreover, $G_{p} \otimes H_{p} \cong I_{p} \otimes G \otimes I_{p} \otimes H \cong I_{p} \otimes I_{p}$ $\otimes G \otimes H \cong I_{p} \otimes G \otimes H$. Since $G \otimes H$ is compact and totally disconnected, Proposition 5.3 shows that $I_{p} \otimes G \otimes H \cong(G \otimes H)_{p}$. Hence $(G \otimes H)_{p} \cong G_{p} \otimes H_{p}$, and $\prod_{p} G_{p} \otimes H_{p}$ is the primary decomposition of $G \otimes H$. (2) follows immediately from (1), and (3) was proven in the course of proving (1). This completes the proof.

Finally, Theorem 5.10 yields, in combination with the corollary to Proposition 5.1 , the following result.

THEOREM 5.11. If $G$ and $H$ are arbitrary compact groups then $G \otimes H$ is a compact totally disconnected group whose p-primary component is $\left(G / G_{0}\right)_{p} \otimes\left(H / H_{0}\right)_{p}$.

VI. Ext and Tor. The standard homological algebra [6] applies in its entirety only to abelian categories. As we have noted in the Introduction, $\mathscr{L}$ is not an abelian category. These difficulties have already been encountered in $\S \S \mathrm{IV}$ and V, where the functors Hom, $\otimes$ and related functors were discussed. They were resolved by restricting the homomorphisms and the corresponding sequences to be proper, and by restricting the domains of definition of the various functors. In this section, we define the functors Ext and Tor as derived functors of Hom and $\bigotimes$ by projective and injective resolutions. Similarly here, the resolutions employed must be proper. The domains of the functors Ext and Tor must be included in those of Hom and $\otimes$ respectively and, in fact, will have to be restricted even further. 
Even so, all the difficulties have not been systematically isolated. It is necessary to make ad hoc arguments at certain places within a proof in order to show that various subgroups are closed and, more particularly, that various homomorphisms are proper. Moreover, this procedure is not always possible in certain of the standard methods of proof of homological algebra, and the results must be gotten from others by dualization. However, with the restrictions on the domains, the admissible homomorphisms, and the resolutions indicated above, all the homological results that one would want are true. Although we do not work out every detail of the theory completely but merely assert that this or that standard homological method works, in one case the work is done in complete detail; namely in the proof of Theorem 6.6.

THEOREM 6.1. Let $G$ and $H$ be in $\mathscr{L}$ and suppose that

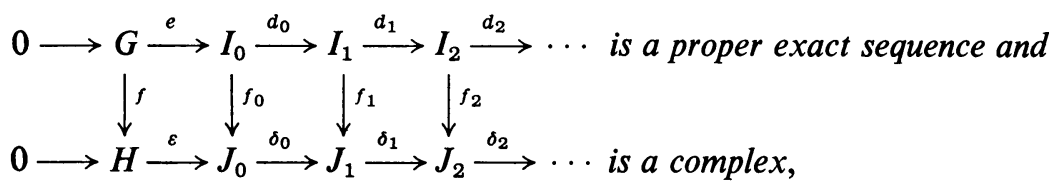

where $J_{n}$ is injective for $n=1,2, \ldots$ Then given $f \in \operatorname{Hom}(G, H)$, there exists, for each integer $n$, an $f_{n} \in \operatorname{Hom}\left(I_{n}, J_{n}\right)$ such that the above diagram is commutative. Moreover, any two such sequences of maps are homotopic in the sense that, if $g_{n}$ is another such sequence of continuous homomorphisms, there exist $h_{n} \in \operatorname{Hom}\left(I_{n}, J_{n-1}\right)$, for $n=1,2, \ldots$, and $h_{0} \in \operatorname{Hom}\left(I_{0}, H\right)$ such that $f_{n}-g_{n}=\delta_{n-1} h_{n}+h_{n+1} d_{n}$, for $n=1,2, \ldots$, and $f_{0}-g_{0}=h_{1} d_{0}+\varepsilon h_{0}$. (Actually, $h_{0}$ may be taken to be 0 .)

Dually, let $G$ and $H \in \mathscr{L}$ and suppose that

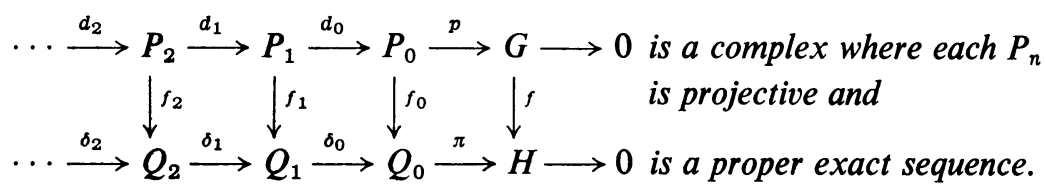

Then, given $f \in \operatorname{Hom}(G, H)$, there exists for each integer $n$, an $f_{n} \in \operatorname{Hom}\left(P_{n}, Q_{n}\right)$ such that the above diagram is commutative. Moreover, any two such sequences of maps are homotopic.

Proof. The proof proceeds along the usual lines (see [6]), except that the assumption that the top row is proper takes care of the presence of topology. The second statement follows from the first by dualization.

COROLlaRY. If $0 \longrightarrow G \stackrel{e}{\longrightarrow} I_{0} \stackrel{d_{0}}{\longrightarrow} I_{1} \stackrel{d_{1}}{\longrightarrow}$ and

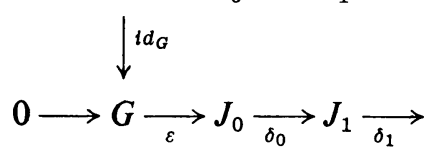

are two injective resolutions of $G$ then, for each $n$, there exists $f_{n} \in \operatorname{Hom}\left(I_{n}, J_{n}\right)$ and $g_{n} \in \operatorname{Hom}\left(J_{n}, I_{n}\right)$ such that $g_{n} f_{n}$ and $f_{n} g_{n}$ are homotopic to $\mathrm{id}_{I_{n}}$ and $\mathrm{id}_{J_{n}}$, respectively.

Dually, any two projective resolutions of a group $G$ are homotopic. 
The proofs are obvious from Theorem 6.1.

In order to define $\operatorname{Ext}^{I}(G, H)$ as a derived functor of $\operatorname{Hom}(G, H)$ by injective resolution of $H$, it is necessary that $G$ in $\mathscr{C}$ and $H$ in $\mathscr{N}$ (see $\S \mathrm{V}$ ). However, since $H$ is to have an injective resolution it is necessary, by Theorem 3.6, that $H$ is in $\mathscr{C}$. Thus we must assume that $G$ in $\mathscr{C}$ and $H$ in $\mathscr{C} \cap \mathscr{N}$.

Definition. A group in $\mathscr{C} \cap \mathscr{N}$ is called elementary.

It follows directly from Theorems 2.4 and 2.5 that $E$ is elementary if and only if $E \cong R^{n} \oplus T^{m} \oplus Z^{s} \oplus F$ where $F$ is a finite group.

Let $E$ be an elementary group and consider injective resolutions of

$$
E: 0 \longrightarrow E \stackrel{e}{\longrightarrow} I_{0} \stackrel{d_{0}}{\longrightarrow} I_{1} \stackrel{d_{1}}{\longrightarrow} I_{2} \stackrel{d_{2}}{\longrightarrow} \cdots
$$

where $I_{n}$ is in $\mathscr{N}$, that is, $I_{n}$ is elementary (Theorem 3.2). We prove the existence of such resolutions shortly. Let $G \in \mathscr{C}$. By the corollary to Theorem 5.3, the induced sequence

$$
0 \longrightarrow \operatorname{Hom}(G, E) \stackrel{e^{*}}{\longrightarrow} \operatorname{Hom}\left(G, I_{0}\right) \stackrel{d_{0}^{*}}{\longrightarrow} \operatorname{Hom}\left(G, I_{1}\right) \stackrel{d_{\mathrm{i}}}{\longrightarrow} \operatorname{Hom}\left(G, I_{2}\right) \stackrel{d_{2}^{*}}{\longrightarrow}
$$

which we denote by $\operatorname{Hom}(G, I(E))$ is proper. Moreover, it is clear that $\operatorname{Hom}(G, I(E))$ is a complex. The sequence obtained from $\operatorname{Hom}(G, I(E))$ by deleting all terms after Hom $\left(G, I_{1}\right)$ is proper exact by Theorem 5.3. Finally, since Hom $(G, I(E))$ is a complex and the homomorphisms are proper, it follows that $d_{n-1}^{*}\left(\operatorname{Hom}\left(G, I_{n-1}\right)\right)$ is a closed subgroup of $\operatorname{Ker} d_{n}^{*}$.

Definition. We denote the $n$th homology group of $\operatorname{Hom}(G, I(E))$ by $\operatorname{Ext}_{n}^{I}(G, E)$. The corollary to Theorem 6.1 shows that the topological groups so defined are independent (to within natural isomorphisms) of the resolution. As usual $\operatorname{Ext}_{n}^{I}(G, E)$ is an additive functor contravariant in $G$ and covariant in $E$.

Since $E$ is elementary, as was remarked above, $E \cong R^{n} \oplus T^{m} \oplus Z^{s} \oplus F$, where $F$ is a finite group and hence is the direct product of a finite number of cyclic groups $Z_{n_{i}}$. As in §III, an injective resolution of $E$ may be found by finding an injective resolution of the factors of $E$ and then taking their direct product.

Injective resolutions of $R, T, Z$, and $Z_{n}$ are the following:

$$
\begin{aligned}
& 0 \rightarrow R \rightarrow R \rightarrow 0 \rightarrow 0 \\
& 0 \rightarrow T \rightarrow T \rightarrow 0 \rightarrow 0 \\
& 0 \rightarrow Z \rightarrow R \rightarrow T \rightarrow 0 \\
& 0 \rightarrow Z_{n} \rightarrow T \rightarrow T \rightarrow 0 .
\end{aligned}
$$

Thus, if $E$ is elementary, there exists an injective resolution

$$
0 \longrightarrow E \stackrel{e}{\longrightarrow} I_{0} \stackrel{d_{0}}{\longrightarrow} I_{1} \stackrel{d_{1}}{\longrightarrow} I_{2} \stackrel{d_{2}}{\longrightarrow} \cdots
$$

where $I_{n}=(0)$ for $n \geqq 2$ and $I_{n}$ is elementary for $n=1,2$. Hence $d_{n}=0$ for $n \geqq 1$ so that $d_{n}^{*}=0$ for $n \geqq 1$. 
Proposition 6.1. Let $G$ be in $\mathscr{C}$ and E be elementary. Then $\operatorname{Ext}_{0}^{I}(G, E) \cong \operatorname{Hom}(G, E)$, $\operatorname{Ext}_{n}^{I}(G, E)=(0)$ for $n \geqq 2$, and $\operatorname{Ext}_{1}^{I}(G, E) \cong \operatorname{Hom}\left(G, I_{1}\right) / d_{0}^{*}\left(\operatorname{Hom}\left(G, I_{0}\right)\right)$, where $d_{0}^{*}$ is the map induced from the standard short resolution of $E$. In particular, $\operatorname{Ext}_{1}^{I}(G, E)$ is a discrete group.

Proof. For $n \geqq 2, \operatorname{Ker} d_{n}^{*}=\operatorname{Hom}\left(G, I_{n}\right)=(0)$, since $I_{n}=(0)$. Thus $\operatorname{Ext}_{n}^{I}(G, E)$ $=(0) . \quad \operatorname{Ext}_{1}^{I}(G, E)=\operatorname{Ker} d_{1}^{*} / d_{0}^{*}\left(\operatorname{Hom}\left(G, I_{0}\right)\right)=\operatorname{Hom}\left(G, I_{1}\right) / d_{0}^{*}\left(\operatorname{Hom}\left(G, I_{0}\right)\right)$. $\operatorname{Ext}_{0}^{I}(G, E)=\operatorname{Ker} d_{0}^{*}=e^{*}(\operatorname{Hom}(G, E)) \cong \operatorname{Hom}(G, E)$, since $0 \rightarrow \operatorname{Hom}(G, E) \rightarrow$ $\operatorname{Hom}\left(G, I_{0}\right) \rightarrow \operatorname{Hom}\left(G, I_{1}\right)$ is a proper exact sequence. Now because $G$ is in $\mathscr{C}$ and $I_{0}$ and $I_{1}$ are in $\mathscr{N}$, a dual version of Theorem 4.10 yields that $d_{0}^{*}: \operatorname{Hom}\left(G, I_{0}\right)$ $\rightarrow \operatorname{Hom}\left(G, I_{1}\right)$ is an open map. Hence $d_{0}^{*}\left(\operatorname{Hom}\left(G, I_{0}\right)\right)$ is open in $\operatorname{Hom}\left(G, I_{1}\right)$ and therefore $\operatorname{Ext}_{1}^{I}(G, E)$ is discrete. This completes the proof of Proposition 6.1.

THEOREM 6.2. If $0 \rightarrow E_{1} \rightarrow E_{2} \rightarrow E_{3} \rightarrow 0$ is a proper exact sequence of elementary groups, and $G$ is in $\mathscr{C}$, then

$$
\begin{aligned}
0 \rightarrow \operatorname{Hom}\left(G, E_{1}\right) \rightarrow & \operatorname{Hom}\left(G, E_{2}\right) \rightarrow \operatorname{Hom}\left(G, E_{3}\right) \\
& \rightarrow \operatorname{Ext}_{1}^{I}\left(G, E_{1}\right) \rightarrow \operatorname{Ext}_{1}^{I}\left(G, E_{2}\right) \rightarrow \operatorname{Ext}_{1}^{I}\left(G, E_{3}\right) \rightarrow 0
\end{aligned}
$$

is a proper exact sequence. In particular, if $0 \rightarrow E \rightarrow I_{0} \rightarrow I_{1} \rightarrow I_{2} \rightarrow \cdots$ is any injective resolution of $E$ by elementary groups, then

$$
0 \rightarrow \operatorname{Hom}(G, E) \rightarrow \operatorname{Hom}\left(G, I_{0}\right) \rightarrow \operatorname{Hom}\left(G, I_{1}\right) \rightarrow \operatorname{Ext}_{1}^{I}(G, E) \rightarrow 0
$$

is a proper exact sequence. Similarly, if $0 \rightarrow G_{1} \rightarrow G_{2} \rightarrow G_{3} \rightarrow 0$ is a proper exact sequence of groups in $\mathscr{C}$ and $E$ is elementary, then

$$
\begin{aligned}
0 \rightarrow \operatorname{Hom}\left(G_{3}, E\right) \rightarrow \operatorname{Hom}\left(G_{2}, E\right) & \rightarrow \operatorname{Hom}\left(G_{1}, E\right) \\
& \rightarrow \operatorname{Ext}_{1}^{I}\left(G_{3}, E\right) \rightarrow \operatorname{Ext}_{1}^{I}\left(G_{2}, E\right) \rightarrow \operatorname{Ext}_{1}^{I}\left(G_{1}, E\right) \rightarrow 0
\end{aligned}
$$

is a proper exact sequence.

Proof. The sequence obtained from 6.1 by deleting all terms after $\operatorname{Hom}\left(G, E_{3}\right)$ is proper exact by Theorem 5.3. Clearly, all maps in 6.1 are continuous. They are trivially proper since $\operatorname{Ext}_{1}^{I}\left(G, E_{i}\right)$ is discrete for $i=1,2,3$. The proof that the sequence is exact follows the usual one in homological algebra [6]. It terminates because $\operatorname{Ext}_{n}^{I}\left(G, E_{i}\right)=(0)$ for $i=1,2,3$ and $n \geqq 2$. This proves that 6.1 is proper exact. The second statement follows from this because $\operatorname{Ext}_{1}^{I}\left(G, I_{0}\right)=(0)$ since $I_{0}$ is injective. The final statement follows from the standard exact sequence for a complex and a subcomplex.

We utilize Proposition 6.1 to compute $\operatorname{Ext}_{1}^{I}$ explicitly.

Theorem 6.3. Let $G$ be in $\mathscr{C}$ and $E$ be elementary. Then

$$
\operatorname{Ext}_{1}^{I}(G, E) \cong\left(C^{\wedge}\right)^{s} \oplus C^{\wedge} / n_{1} C^{\wedge} \oplus \cdots \oplus C^{\wedge} / n_{k} C^{\wedge}
$$

where $C$ is the maximum compact subgroup of $G$ and $s, n_{1}, \ldots, n_{k}$ are respectively the rank and torsion numbers of $E / E_{0}$. In particular, $\operatorname{Ext}_{1}^{I}(G, E) \cong \operatorname{Ext}_{1}^{I}\left(C, E / E_{0}\right)$. 
Proof. First we compute some special cases. If $E=Z$, the injective resolution

$$
0 \longrightarrow Z \longrightarrow R \stackrel{\pi}{\longrightarrow} T \longrightarrow 0
$$

of $Z$ show that $\operatorname{Ext}_{1}^{I}(R, Z)=(0)$, since $(R, \pi)$ is a covering group of $T$ and $R$ is simply connected. Also $\operatorname{Ext}_{1}^{I}(Z, Z)=(0)$, clearly. If $C$ is compact then $\operatorname{Ext}_{1}^{I}(C, Z)$ $=C^{\wedge}$, since $R$ has no nontrivial compact subgroups.

Now $G \cong R^{n} \oplus Z^{m} \oplus C$, because $G$ is compactly generated. Since Ext $_{1}^{I}$ is additive, it follows that $\operatorname{Ext}_{1}^{I}(G, Z)=C^{\wedge}$ where $C$ is the maximum compact subgroup of $G$. If $E=Z_{n}$, the cyclic group of order $n$, the injective resolution

$$
0 \longrightarrow Z_{n} \longrightarrow T \stackrel{\pi}{\longrightarrow} T \longrightarrow 0
$$

of $Z_{n}$ shows that $\operatorname{Ext}_{1}^{I}\left(R, Z_{n}\right)=(0)$, since $(T, \pi)$ is a covering group of $T$ and $R$ is simply connected. Also $\operatorname{Ext}_{1}^{I}\left(Z, Z_{n}\right)=(0)$, since $T$ is divisible. If $C$ is compact then, obviously, $\operatorname{Ext}_{1}^{I}\left(C, Z_{n}\right) \cong C^{\wedge} / n C^{\wedge}$. Consequently, $\operatorname{Ext}_{1}^{I}\left(G, Z_{n}\right) \cong C^{\wedge} / n C^{\wedge}$, where $C$ is the maximum compact subgroup of $G$.

Finally, take $E=R^{n} \oplus T^{m} \oplus Z^{s} \oplus F$. Then $\operatorname{Ext}_{1}^{I}\left(G, E_{0}\right)=(0)$ since $E_{0}$ is injective. Now, by additivity, $\operatorname{Ext}_{1}^{I}(G, E) \cong \operatorname{Ext}_{1}^{I}\left(G, E_{0}\right) \oplus \operatorname{Ext}_{1}^{I}(G, Z)^{s} \oplus \operatorname{Ext}_{1}^{I}(G, F)$. The result follows.

COROLLARY. If (1) the maximum compact subgroup of $G$ is $(0)$, or

(2) $E$ is connected, or

(3) $G$ is torsion free and $E / E_{0}$ is finite then $\operatorname{Ext}_{1}^{I}(G, E)=(0)$.

Proof. (1) is clear from Theorem 6.3. (2) has already been seen in the proof of Theorem 6.3. To prove (3), suppose $E / E_{0}$ is finite, so that $s=0$. Since $G$ is torsion free, so is $C$. Hence $C^{\wedge}$ is divisible, by Theorem 2.9 , and $C^{\wedge} / n_{i} C^{\wedge}=(0)$ for $i=1, \ldots, k$. The result follows.

Let $G$ and $H$ be in $\mathscr{L}$. We define $\operatorname{Ext}^{P}(G, H)$ as a derived functor of $\operatorname{Hom}(G, H)$ by taking projective resolutions of $G$. As above, it is clear that $G$ must be an elementary group which we denote by $E$, and $H$ in $\mathscr{N}$. Consider projective resolutions

$$
\stackrel{d_{2}}{\longrightarrow} P_{2} \stackrel{d_{1}}{\longrightarrow} P_{1} \stackrel{d_{0}}{\longrightarrow} P_{0} \stackrel{\pi}{\longrightarrow} E \longrightarrow 0
$$

of $E$ where $P_{n} \in \mathscr{C}$, i.e., is elementary (Theorem 3.3). Since a group is in $\mathscr{C}$ if and only if its dual is in $\mathscr{N}$, by Theorem 2.5 , Corollary 1 , it follows that $E$ is elementary if and only if $E^{\wedge}$ is elementary. Hence the existence of such resolutions follows by dualizing the resolution constructed on page 394 . By the Corollary to Theorem 5.3, the induced sequence

$$
\begin{aligned}
0 \longrightarrow \operatorname{Hom}(E, H) \stackrel{\pi^{*}}{\longrightarrow} \operatorname{Hom}\left(P_{0}, H\right) & \stackrel{d_{\dot{0}}^{*}}{\longrightarrow} \operatorname{Hom}\left(P_{1}, H\right) \stackrel{d^{\mathbf{1}}}{\longrightarrow} \operatorname{Hom}\left(P_{2}, H\right) \stackrel{d_{2}^{*}}{\longrightarrow} \cdots
\end{aligned}
$$

which we denote by $\operatorname{Hom}(P(E), H)$ is proper. Obviously, $\operatorname{Hom}(P(E), H)$ is a complex. The sequence obtained from $\operatorname{Hom}(P(E), H)$ by deleting all terms after $\operatorname{Hom}\left(P_{1}, H\right)$ is proper exact, by Theorem 5.3. Finally, since $\operatorname{Hom}(P(E), H)$ is a 
complex and the homomorphisms are proper, it follows that $d_{n-1}^{*}\left(\operatorname{Hom}\left(P_{n-1}, H\right)\right)$ is a closed subgroup of $\operatorname{Ker} d_{n}^{*}$.

Definition. We denote the $n$th homology group of $\operatorname{Hom}(P(E), H)$ by $\operatorname{Ext}_{n}^{P}(E, H)$. The corollary to Theorem 6.1 shows that the topological groups so defined are independent (to within natural isomorphisms) of the resolution. As usual, $\operatorname{Ext}_{n}^{P}(E, H)$ is an additive functor contravariant in $E$ and covariant in $H$.

A standard argument [6] utilizing the appropriate double complex shows that on their common domain, i.e., pairs $(G, H)$ where both $G$ and $H$ are elementary, $\operatorname{Ext}_{n}^{I}(G, H)$ and $\operatorname{Ext}_{n}^{P}(G, H)$ are naturally isomorphic.

REMARK. For $G$ in $\mathscr{C}$ and $E$ elementary, the fact that $\operatorname{Ext}_{1}^{I}(G, E)$ and $\operatorname{Ext}_{1}^{I}\left(C, E / E_{0}\right)$ are naturally isomorphic follows from the exactness of the two homology sequences. In fact, consider the proper exact sequence (0) $\rightarrow E_{0} \rightarrow E \rightarrow$ $E / E_{0} \rightarrow(0)$. It follows that $\rightarrow \operatorname{Ext}_{1}^{I}\left(G, E_{0}\right) \rightarrow \operatorname{Ext}_{1}^{I}(G, E) \rightarrow \operatorname{Ext}_{1}^{I}\left(G, E / E_{0}\right) \rightarrow(0)$ is proper exact. But since $E_{0}$ is injective, $\operatorname{Ext}_{1}^{I}\left(G, E_{0}\right)=(0)$. Therefore $\operatorname{Ext}_{1}^{I}(G, E)$ $\cong \operatorname{Ext}_{1}^{I}\left(G, E / E_{0}\right)$. On the other hand, consider the sequence $(0) \rightarrow C \rightarrow G \rightarrow$ $G / C \rightarrow(0)$. Then $\rightarrow \operatorname{Ext}_{1}^{I}\left(G / C, E / E_{0}\right) \rightarrow \operatorname{Ext}_{1}^{I}\left(G, E / E_{0}\right) \rightarrow \operatorname{Ext}_{1}^{I}\left(C, E / E_{0}\right) \rightarrow(0)$ is proper exact. Since both $G / C$ and $E / E_{0}$ are elementary, $\operatorname{Ext}_{1}^{I}\left(G / C, E / E_{0}\right)$ $\cong \operatorname{Ext}_{1}^{P}\left(G / C, E / E_{0}\right)$. But since $G / C$ is a projective it follows that $\operatorname{Ext}_{1}^{I}\left(G / C, E / E_{0}\right)$ $=(0)$. Hence $\operatorname{Ext}_{1}^{I}\left(G, E / E_{0}\right) \cong \operatorname{Ext}_{1}^{I}\left(C, E / E_{0}\right)$ so that $\operatorname{Ext}_{1}^{I}(G, E) \cong \operatorname{Ext}_{1}^{I}\left(C, E / E_{0}\right)$. The corresponding facts about the other functors can be proven similarly.

THEOREM 6.4. If E is elementary and $H$ is in $\mathscr{N}$ then $\operatorname{Ext}_{n}^{P}(E, H)$ and $\operatorname{Ext}_{n}^{I}\left(H^{\wedge}, E^{\wedge}\right)$ are naturally isomorphic.

Proof. Clearly, $\operatorname{Ext}_{0}^{P}(E, H) \cong \operatorname{Hom}(E, H)$. So if $n=0$, the theorem follows from the fact that $\operatorname{Hom}(E, H)$ is naturally isomorphic with $\operatorname{Hom}\left(H^{\wedge}, E^{\wedge}\right)$. In general, $\operatorname{Ext}_{n}^{P}(E, H)=\operatorname{Ker} d_{n}^{*} / d_{n-1}^{*}\left(\operatorname{Hom}\left(P_{n-1}, H\right)\right)$. Moreover, $E^{\wedge}$ is elementary, $H^{\wedge}$ is in $\mathscr{C}$, and

$$
0 \longrightarrow E^{\wedge} \stackrel{\pi^{\wedge}}{\longrightarrow} P_{0}^{\wedge} \stackrel{d_{0}^{\hat{0}}}{\longrightarrow} P_{1}^{\wedge} \stackrel{d_{1}}{\longrightarrow} P_{2}^{\wedge} \stackrel{d_{2}}{\longrightarrow} \cdots
$$

is an injective resolution of $E^{\wedge}$, which is admissible in defining $\operatorname{Ext}_{n}^{I}\left(H^{\wedge}, E^{\wedge}\right)$. Thus $\operatorname{Ext}_{n}^{I}\left(H^{\wedge}, E^{\wedge}\right) \cong \operatorname{Ker}\left(d_{n}^{\wedge}\right)^{*} /\left(d_{n-1}^{\wedge}\right)^{*}\left(\operatorname{Hom}\left(H^{\wedge}, P_{n-1}\right)\right)$, where homology is computed from the complex

$$
\begin{aligned}
0 \longrightarrow \operatorname{Hom}\left(H^{\wedge}, E^{\wedge}\right) \stackrel{\left(\pi^{\wedge}\right)^{*}}{\longrightarrow} & \operatorname{Hom}\left(H^{\wedge}, P_{0}^{\wedge}\right) \\
& \stackrel{\left(d_{\hat{0}}^{*}\right)^{*}}{\longrightarrow} \operatorname{Hom}\left(H^{\wedge}, P_{1}^{\wedge}\right) \stackrel{\left(d_{1}\right)^{*}}{\longrightarrow} \operatorname{Hom}\left(H^{\wedge}, P_{2}^{\wedge}\right) \stackrel{\left(d_{2}\right)^{*}}{\longrightarrow} .
\end{aligned}
$$

Now for each $n$, Hom $\left(P_{n}, H\right)$ and $\operatorname{Hom}\left(H^{\wedge}, P_{n}^{\wedge}\right)$ are isomorphic in a functorial way. Under this identification, one sees easily that $\operatorname{Ker}\left(d_{n}^{\wedge}\right)^{*}=\operatorname{Ker} d_{n}^{*}$ and $d_{n-1}^{*}\left(\operatorname{Hom}\left(P_{n-1}, H\right)\right)=\left(d_{n-1}^{\wedge}\right) *\left(\operatorname{Hom}\left(H, P_{n-1}^{\wedge}\right)\right)$. This completes the proof.

Corollary 1. If $0 \rightarrow E_{1} \rightarrow E_{2} \rightarrow E_{3} \rightarrow 0$ is a proper exact sequence of elementary groups and $H$ is in $\mathcal{N}$, then the following is a proper exact sequence.

$$
\begin{aligned}
& 0 \rightarrow \operatorname{Hom}\left(E_{3}, H\right) \rightarrow \operatorname{Hom}\left(E_{2}, H\right) \rightarrow \operatorname{Hom}\left(E_{1}, H\right) \\
& \rightarrow \operatorname{Ext}_{1}^{P}\left(E_{3}, H\right) \rightarrow \operatorname{Ext}_{1}^{P}\left(E_{2}, H\right) \rightarrow \operatorname{Ext}_{1}^{P}\left(E_{1}, H\right) \rightarrow 0 .
\end{aligned}
$$


In particular, if $\cdots \rightarrow P_{2} \rightarrow P_{1} \rightarrow P_{0} \rightarrow E \rightarrow 0$ is any projective resolution of $E$ by elementary groups, then the following is a proper exact sequence.

$0 \rightarrow \operatorname{Hom}(E, H) \rightarrow \operatorname{Hom}\left(P_{0}, H\right) \rightarrow \operatorname{Hom}\left(P_{1}, H\right) \rightarrow \operatorname{Ext}_{1}(E, H) \rightarrow 0$.

Similarly, if $0 \rightarrow H_{1} \rightarrow H_{2} \rightarrow H_{3} \rightarrow 0$ is a proper exact sequence of groups in $\mathscr{N}$ and $E$ is elementary, then

$$
\begin{aligned}
0 \rightarrow \operatorname{Hom}\left(E, H_{1}\right) \rightarrow \operatorname{Hom}\left(E, H_{2}\right) & \rightarrow \operatorname{Hom}\left(E, H_{3}\right) \\
& \rightarrow \operatorname{Ext}_{1}^{P}\left(E, H_{1}\right) \rightarrow \operatorname{Ext}_{1}^{P}\left(E, H_{2}\right) \rightarrow \operatorname{Ext}_{1}^{P}\left(E, H_{3}\right) \rightarrow 0
\end{aligned}
$$

is a proper exact sequence.

Corollary 2. If $E$ is elementary and $H$ is in $\mathscr{N}$ then $\operatorname{Ext}_{0}^{P}(E, H) \cong \operatorname{Hom}(E, H)$.

$$
\operatorname{Ext}_{1}^{P}(E, H) \cong\left(H / H_{0}\right)^{m} \oplus\left(H / H_{0}\right) / n_{1}\left(H / H_{0}\right) \oplus \cdots \oplus\left(H / H_{0}\right) / n_{k}\left(H / H_{0}\right)
$$

where $m$ is the dimension of the maximal toral subgroup of $E$, and $n_{1}, \ldots, n_{k}$ are torsion numbers of $E / E_{0}$. In particular, $\operatorname{Ext}_{1}^{P}(E, H)$ is a discrete group and is isomorphic with $\operatorname{Ext}_{1}^{P}$ (maximum compact subgroup of $\left.E, H / H_{0}\right)$. Finally $\operatorname{Ext}_{n}^{P}(E, H)$ $=(0)$ for $n \geqq 2$.

The proofs of Corollaries 1 and 2 follow from Theorem 6.4 and the corresponding facts about $\operatorname{Ext}_{n}^{I}$ by dualization.

Corollary 3. If (1) $H$ is connected, or

(2) $E$ is torsion free, or

(3) $E$ has no toral subgroup and $H$ is divisible, then $\operatorname{Ext}_{1}^{P}(E, H)=(0)$.

Proof. This follows directly from Corollary 2.

Let $G$ and $H$ be in $\mathscr{L}$. We define Tor $(G, H)$ as a derived functor of $G \otimes H$ by taking projective resolutions of $H$. As above, we must assume that $G$ is in $\mathscr{C}$ and $H$ is elementary. We denote $H$ by $E$. As above, consider projective resolutions

$$
\stackrel{d_{2}}{\longrightarrow} P_{2} \stackrel{d_{1}}{\longrightarrow} P_{1} \stackrel{d_{0}}{\longrightarrow} P_{0} \stackrel{\pi}{\longrightarrow} E \longrightarrow 0
$$

of $E$, where $P_{n}$ is elementary. It follows from the corollary to Theorem 5.3 that the induced sequence

$$
\stackrel{d_{\mathbf{2}}}{\longrightarrow} G \otimes P_{2} \stackrel{d_{\mathbf{i}}}{\longrightarrow} G \otimes P_{1} \stackrel{d_{0}^{\dot{0}}}{\longrightarrow} G \otimes P_{0} \stackrel{\pi^{*}}{\longrightarrow} G \otimes E \longrightarrow 0,
$$

which we denote by $G \otimes P(E)$, is proper. Obviously, $G \otimes P(E)$ is a complex. The sequence obtained from $G \otimes P(E)$ by deleting all terms before $G \otimes P_{1}$ is proper exact, by Theorem 5.3. Finally, since $G \otimes P(E)$ is a complex and the homomorphisms are proper, it follows that $d_{n}^{*}\left(G \otimes P_{n+1}\right)$ is a closed subgroup of $\operatorname{Ker} d_{n-1}^{*}$.

Definition. We denote the $n$th homology group of $G \otimes P(E)$ by $\operatorname{Tor}_{n}(G, E)$. The corollary to Theorem 6.1 shows that the topological groups so defined are 
independent (to within natural isomorphisms) of the resolution. As usual, $\operatorname{Tor}_{n}(G, E)$ is an additive functor covariant in both variables.

TheOREM 6.5. If $G$ is in $\mathscr{C}$ and $E$ is elementary, then $\operatorname{Tor}_{n}(G, E)$ is naturally isomorphic with $\left(\operatorname{Ext}_{n}^{I}\left(G, E^{\wedge}\right)\right)^{\wedge}$.

Proof. Tor To $_{0}(G, E)=\left(G \otimes P_{0}\right) / d_{0}^{*}\left(G \otimes P_{1}\right) \cong\left(G \otimes P_{0}\right) /$ Ker $\pi^{*} \cong G \otimes E$, since $G \otimes P_{1} \rightarrow G \otimes P_{0} \rightarrow G \otimes E \rightarrow 0$ is a proper exact sequence. Since $E^{\wedge}$ is elementary and $G$ is in $\mathscr{C}, \operatorname{Ext}_{0}^{I}\left(G, E^{\wedge}\right)$ is naturally isomorphic with $\operatorname{Hom}\left(G, E^{\wedge}\right)$, by Proposition 6.1. As was shown in $\S \mathrm{IV}, G \otimes E$ is naturally isomorphic with $\operatorname{Hom}\left(\left(G, E^{\wedge}\right)\right)^{\wedge}$. This proves the theorem in the case $n=0$. Now, $E^{\wedge}$ is elementary, and

$$
0 \longrightarrow E^{\wedge} \stackrel{\pi^{\wedge}}{\longrightarrow} P_{0} \stackrel{d_{\hat{0}}}{\longrightarrow} P_{1} \stackrel{d_{\hat{1}}}{\longrightarrow} P_{2} \stackrel{d_{\hat{2}}}{\longrightarrow}
$$

is an injective resolution of $E^{\wedge}$, which is admissible in defining $\operatorname{Ext}_{n}^{I}\left(G, E^{\wedge}\right)$. Hence, $\operatorname{Ext}_{n}^{I}\left(G, E^{\wedge}\right) \cong \operatorname{Ker}\left(d_{n}^{\wedge}\right)^{*} /\left(d_{n-1}^{\wedge}\right)^{*}\left(\operatorname{Hom}\left(G, P_{n-1}^{\wedge}\right)\right)$ for $n \geqq 1$, where homology is computed from the complex

$$
0 \longrightarrow \operatorname{Hom}\left(G, E^{\wedge}\right) \stackrel{\left(\pi^{\wedge}\right)^{*}}{\longrightarrow} \operatorname{Hom}\left(G, P_{0}^{\wedge}\right) \stackrel{\left(d_{\hat{0}}\right)^{\bullet}}{\longrightarrow} \operatorname{Hom}\left(G, P_{1}^{\wedge}\right) \stackrel{\left(d_{\hat{1}}\right)^{*}}{\longrightarrow} \cdots
$$

However, the results of $\S \mathrm{V}$ show that this sequence is precisely

$$
0 \longrightarrow(G \otimes E)^{\wedge} \stackrel{\left(\pi^{*}\right) \wedge}{\longrightarrow}\left(G \otimes P_{0}\right)^{\wedge} \stackrel{\left(d_{0}^{*}\right)^{\wedge}}{\longrightarrow}\left(G \otimes P_{1}\right)^{\wedge} \stackrel{\left(d_{\mathrm{i}}^{*}\right)^{\wedge}}{\longrightarrow} \cdots .
$$

The theorem now follows from Theorem 2.2.

COROLlaRY 1. If $0 \rightarrow E_{1} \rightarrow E_{2} \rightarrow E_{3} \rightarrow 0$ is a proper exact sequence of elementary groups and $G$ is in $\mathscr{C}$ then the following is a proper exact sequence.

$0 \rightarrow \operatorname{Tor}_{1}\left(G, E_{1}\right) \rightarrow \operatorname{Tor}_{1}\left(G, E_{2}\right) \rightarrow \operatorname{Tor}_{1}\left(G, E_{3}\right)$

$$
\rightarrow G \otimes E_{1} \rightarrow G \otimes E_{2} \rightarrow G \otimes E_{3} \rightarrow 0 .
$$

If $\cdots \rightarrow P_{2} \rightarrow P_{1} \rightarrow P_{0} \rightarrow E \rightarrow 0$ is any projective resolution of $E$ by elementary groups, then $0 \rightarrow$ Tor $_{1}(G, E) \rightarrow G \otimes P_{1} \rightarrow G \otimes P_{0} \rightarrow G \otimes E \rightarrow 0$ is a proper exact sequence. Similarly, if $0 \rightarrow G_{1} \rightarrow G_{2} \rightarrow G_{3} \rightarrow 0$ is a proper exact sequence of groups in $\mathscr{C}$ and $E$ is elementary, then

$$
\begin{aligned}
0 \rightarrow \operatorname{Tor}_{1}\left(G_{1}, E\right) \rightarrow \operatorname{Tor}_{1}\left(G_{2}, E\right) \rightarrow & \operatorname{Tor}_{1}\left(G_{3}, E\right) \\
& \rightarrow G_{1} \otimes E \rightarrow G_{2} \otimes E \rightarrow G_{3} \otimes E \rightarrow 0
\end{aligned}
$$

is a proper exact sequence.

Corollary 2. Let $G$ be in $\mathscr{C}$ and $E$ be elementary. Then $\operatorname{Tor}_{0}(G, E) \cong G \otimes E$. Tor $_{1}(G, E) \cong(C)^{m} \oplus C\left[n_{1}\right] \oplus \cdots \oplus C\left[n_{k}\right]$, where $C$ is the maximum compact subgroup of $G, m$ is the toral dimension of $E$, and $n_{1}, \ldots, n_{k}$ are the torsion numbers of $E / E_{0}$. Here $C[n]$ denotes the elements of $C$ whose order divides $n$. In particular, Tor $_{1}(G, E)$ is a compact group isomorphic with Tor $_{1}(C$, maximum compact subgroup of E). Finally $\operatorname{Tor}_{n}(G, E)=(0)$ for $n \geqq 2$. 
The proofs of Corollary 1 and 2 follow from Theorem 6.5 and the corresponding facts about $\operatorname{Ext}_{n}^{I}$ by dualization.

Corollary 3. Let $G$ be in $\mathscr{C}$ and $E$ be elementary. If

(1) the maximum compact subgroup of $G=(0)$, or

(2) $E$ is torsion free, or

(3) $G$ is torsion free and $E$ has no toral subgroup, then $\operatorname{Tor}_{1}(G, E)=(0)$.

Proof. This follows immediately from Corollary 2.

Definition. Let $G$ and $H$ be in $\mathscr{L}$. An extension of $H$ by $G$ is a short proper exact sequence

$$
0 \longrightarrow H \stackrel{f}{\longrightarrow} Y \stackrel{g}{\longrightarrow} G \longrightarrow 0
$$

where $Y$ is an abelian topological group. By a theorem of Gleason, [9] or [4], $Y$ is in $\mathscr{L}$.

Definition. Two extensions

and

$$
0 \longrightarrow H \stackrel{f}{\longrightarrow} Y \stackrel{g}{\longrightarrow} G \longrightarrow 0
$$

$$
0 \longrightarrow H \stackrel{f^{\prime}}{\longrightarrow} Y^{\prime} \stackrel{g^{\prime}}{\longrightarrow} G \longrightarrow 0
$$

of $H$ by $G$ are equivalent if there exists a proper homomorphism $h$, making the following diagram commutative.

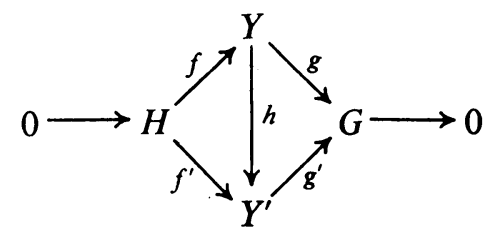

Since $h$ is automatically bijective, $h$ is a topological group isomorphism, so that this is an equivalence relation.

Definition. Denote by $\mathscr{E}(G, H)$ the set of equivalence classes of extensions of $H$ by $G$.

THEOREM 6.6. (1) If $G$ is in $\mathscr{C}$ and $E$ is elementary, then there is a natural bijective correspondence between $\mathscr{E}(G, E)$ and $\operatorname{Ext}_{1}^{I}(G, E)$.

(2) If $E$ is elementary and $H$ is in $\mathscr{N}$, then there is a natural bijective correspondence between $\mathscr{E}(E, H)$ and $\operatorname{Ext}_{1}^{P}(E, H)$.

Moreover, these correspondences transform the Baer composition of extensions to the group operation in $\mathrm{Ext}_{1}$ so that they are actually group isomorphisms.

Whereas in the case of abstract groups, the usual homological proofs of both (1) and its dual (2) work, in the continuous case there are difficulties connected with (2) and it must be gotten from (1) by dualization. Since the ${ }^{\wedge}$ gives a bijective correspondence between group extensions $0 \rightarrow E \rightarrow Y \rightarrow G \rightarrow 0$, where $E$ is elementary and $G$ is in $\mathscr{C}$ and $0 \rightarrow G^{\wedge} \rightarrow Y^{\wedge} \rightarrow E^{\wedge} \rightarrow 0$ where $G^{\wedge}$ is in $\mathscr{N}$ and $E^{\wedge}$ is elementary, and since if $h$ is an equivalence between extensions of $E$ by $G$, 
then $h^{\wedge}$ is an equivalence between their duals, it follows that there is a natural bijective correspondence between $\mathscr{E}(G, E)$ and $\mathscr{E}\left(E^{\wedge}, G^{\wedge}\right)$ for $G$ in $\mathscr{C}$ and $E$ elementary. On the other hand, by Theorem $6.4, \operatorname{Ext}_{1}^{I}(G, E)$ and $\operatorname{Ext}_{1}^{P}\left(E^{\wedge}, G^{\wedge}\right)$ are naturally isomorphic under the same assumptions. Thus, (2) follows from (1) by dualization. Hence it suffices to prove Part 1 of Theorem 6.6.

Let $0 \longrightarrow E \stackrel{f}{\longrightarrow} Y \stackrel{g}{\longrightarrow} G \longrightarrow 0$ be an extension where $E$ $\downarrow^{\operatorname{id}_{E}} \quad \downarrow^{l_{0}} \quad \downarrow^{l_{1}} \quad$ is elementary and $G$ is in $\mathscr{C}$.

Let $0 \longrightarrow E \underset{e}{\longrightarrow} I_{0} \underset{d}{\longrightarrow} I_{1} \longrightarrow 0$ be a short injective resolution of $E$ where $I_{0}$ and $I_{1}$ are elementary. By Theorem 6.1, choose $l_{0} \in \operatorname{Hom}\left(Y, I_{0}\right)$ and $l_{1} \in \operatorname{Hom}\left(G, I_{1}\right)$ so that the above diagram is commutative. By Theorem 6.2 , the sequence

$$
0 \longrightarrow \operatorname{Hom}(G, E) \longrightarrow \operatorname{Hom}\left(G, I_{0}\right) \stackrel{\delta}{\longrightarrow} \operatorname{Hom}\left(G, I_{1}\right) \stackrel{\gamma}{\longrightarrow} \operatorname{Ext}_{1}^{I}(G, E) \longrightarrow 0
$$

is proper exact. Define the continuous homomorphism $\lambda_{1}: \operatorname{Hom}(G, G) \rightarrow$ Hom $\left(G, I_{1}\right)$ by $\lambda_{1}(\xi)=l_{1} \circ \xi$. Then $\gamma \lambda_{1}\left(\operatorname{id}_{G}\right) \in \operatorname{Ext}_{1}^{I}(G, E)$ and is clearly independent of the representative of $\mathscr{E}(G, E)$, thus giving a map from $\mathscr{E}(G, E) \rightarrow$ $\operatorname{Ext}_{1}^{I}(G, E)$.

Now, given any $l_{1} \in \operatorname{Hom}\left(G, I_{1}\right)$ we construct an extension

$$
0 \longrightarrow E \stackrel{f_{l_{1}}}{\longrightarrow} Y_{l_{1}} \stackrel{g_{l_{1}}}{\longrightarrow} G \longrightarrow 0
$$

as follows. Form the direct sum $G \oplus I_{0}$ and define the continuous homomorphism $\theta_{l_{1}}: G \oplus I_{0} \rightarrow I_{1}$ by $\theta_{l_{1}}\left(x, x_{0}\right)=-l_{1}(x)+d\left(x_{0}\right)$. Let $Y_{l_{1}}=\operatorname{Ker} \theta_{l_{1}}$. It follows from Corollary 1 of Theorem 2.5 and Proposition 2.6 that $G \oplus I_{0}$ is in $\mathscr{C}$. Since $\theta_{l_{1}}$ is continuous, $Y_{l_{1}}$ is a closed subgroup of $G \oplus I_{0}$ and therefore $Y_{l_{1}}$ is in $\mathscr{C}$ by Theorem 2.6. Define $f_{l_{1}}: E \rightarrow Y_{l_{1}}$ by $f_{l_{1}}(u)=(0, e(u))$ for $u \in E$. Now, $(0, e(u)) \in Y_{l_{1}}$ since $\theta_{l_{1}}(0, e(u))=-l_{1}(0)+d(e(u))=0+0$. Since $e$ is a proper monomorphism, so is $f_{l_{1}}$. Define $g_{l_{1}}: Y_{l_{1}} \rightarrow G$ by $g_{l_{1}}\left(x, x_{0}\right)=x$, i.e., $g_{l_{1}}$ is the restriction to $Y_{l_{1}}$ of the projection $G \oplus I_{0} \rightarrow G$. Hence, $g_{l_{1}} \in \operatorname{Hom}\left(Y_{l_{1}}, G\right)$. Moreover, Ker $g_{l_{1}}=Y_{l_{1}} \cap I_{0}$ $=(0)+\operatorname{Ker} d=(0)+e(E)=f_{l_{1}}(E)$. Furthermore,

$$
g_{l_{1}}\left(Y_{l_{1}}\right)=\left\{x: x \in G \text { and } l_{1}(x) \in d\left(I_{0}\right)=I_{1}\right\}=G,
$$

so that $g_{l_{1}}$ is a continuous epimorphism. Since $Y_{l_{1}}$ is in $\mathscr{C}, g_{l_{1}}$ is an open map. Thus,

$$
0 \longrightarrow E \stackrel{f_{l_{1}}}{\longrightarrow} Y_{l_{1}} \stackrel{g_{l_{1}}}{\longrightarrow} G \longrightarrow 0
$$

is an extension. Now define $l_{0}: Y_{l_{1}} \rightarrow I_{0}$ by $l_{0}\left(x, x_{0}\right)=x_{0}$. Since $l_{0}$ is the restriction of a continuous homomorphism, $l_{0} \in \operatorname{Hom}\left(Y_{l_{1}}, I_{0}\right)$. It is immediate that $l_{0} \circ f_{l_{1}}$ $=e \circ \mathrm{id}_{E}$ and $l_{1} \circ g_{l_{1}}=d \circ l_{0}$, so that the following diagram is commutative.

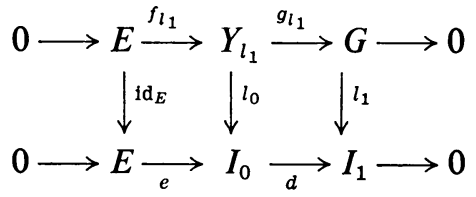


Since $\gamma$ is surjective, this implies that the map $\mathscr{E}(G, E) \rightarrow \operatorname{Ext}_{1}^{I}(G, E)$ is surjective. Now, let

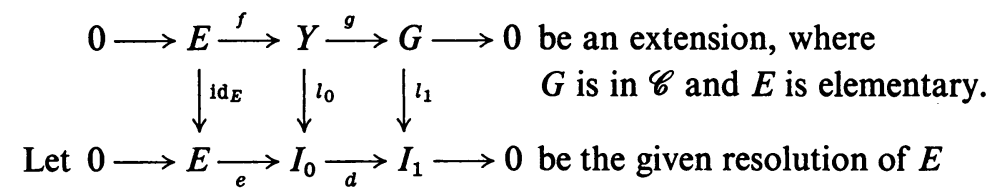

and let $l_{0}, l_{1}$ be extensions of $\mathrm{id}_{E}$. Form

$$
0 \longrightarrow E \stackrel{f_{l_{1}}}{\longrightarrow} Y_{l_{1}} \stackrel{g_{l_{1}}}{\longrightarrow} G \longrightarrow 0
$$

as above. Then

$$
0 \longrightarrow E \stackrel{f_{l_{1}}}{\longrightarrow} Y_{l_{1}} \stackrel{g_{l_{1}}}{\longrightarrow} G \longrightarrow 0
$$

and

$$
0 \longrightarrow E \stackrel{f}{\longrightarrow} Y \stackrel{g}{\longrightarrow} G \longrightarrow 0
$$

are equivalent. To see this, define $h: Y \rightarrow G \oplus I_{0}$ by $h(y)=\left(g(y), l_{0}(y)\right)$. Then $h$ is a continuous homomorphism since $g$ and $l_{0}$ are. If $y \in Y$, then $\theta_{l_{1}}(h(y))$ $=-l_{1}(g(y))+d\left(l_{0}(y)\right)=0$ since $d l_{0}=l_{1} g$. Thus, $h(Y) \subset Y_{l_{1}}$. One sees easily that $h f=f_{l_{1}}$ and $g_{l_{1}} h=g$. Clearly, $h$ is bijective. Since the given extension is proper exact, $Y$ is in $\mathscr{C}$ by Theorem 2.6. Hence $h$ is open, by the Open Mapping Theorem, and the extensions are equivalent.

Let

$$
0 \longrightarrow E \stackrel{f}{\longrightarrow} Y \stackrel{g}{\longrightarrow} G \longrightarrow 0 \text { and } 0 \longrightarrow E \stackrel{f^{\prime}}{\longrightarrow} Y^{\prime} \stackrel{g^{\prime}}{\longrightarrow} G \longrightarrow 0
$$

be two extensions. Form the respective $l_{1}$ and $l_{1}^{\prime}$. Suppose they map into the same cohomology class of $\operatorname{Ext}_{1}^{I}(G, E)$, namely that $\gamma \lambda_{1}\left(\mathrm{id}_{G}\right)=\gamma \lambda_{1}^{\prime}\left(\mathrm{id}_{G}\right)$. Then $\gamma l_{1}=\gamma l_{1}^{\prime}$ so that $l_{1}-l_{1}^{\prime} \in \operatorname{Ker} \gamma$. Since $\operatorname{Ker} \gamma=\delta\left(\operatorname{Hom}\left(G, I_{0}\right)\right)$, it follows that $l_{1}-l_{1}^{\prime}=d \xi$ for some $\xi \in \operatorname{Hom}\left(G, I_{0}\right)$.

Define $\alpha: G \oplus I_{0} \rightarrow G \oplus I_{0}$ by $\alpha\left(x, x_{0}\right)=\left(x, \xi(x)+x_{0}\right)$. Because $\xi$ is a continuous homomorphism, so is $\alpha$. Obviously, $\alpha$ is bijective. Since $G \oplus I_{0}$ is in $\mathscr{C}, \alpha$ is a topological group automorphism, by the Open Mapping Theorem.

Now form $Y_{l_{1}}$ and $Y_{l_{1}^{\prime}}$ as above. We show that $\alpha\left(Y_{l_{1}}\right)=Y_{l_{1}^{\prime}}$. Now $\alpha\left(Y_{l_{1}}\right)$ $=\left\{\alpha\left(x, x_{0}\right): l_{1}(x)=d\left(x_{0}\right)\right\}$. But $l_{1}(x)=l_{1}^{\prime}(x)+d \xi(x)$ for each $x \in G$. Thus,

$$
\alpha\left(Y_{l_{1}}\right)=\left\{\left(x, \xi(x)+x_{0}\right): l_{1}^{\prime}(x)+d \xi(x)=d\left(x_{0}\right)\right\}
$$

which in turn equals $\left\{\left(x, x_{0}-\xi(x)\right): l_{1}^{\prime}(x)=d\left(x_{0}-\xi(x)\right)\right\}$. Since as $x_{0}$ ranges over $I_{0}$, so does $x_{0}-\xi(x)$, the result follows. Hence $\alpha \mid Y_{l_{1}}$ is a topological group isomorphism $Y_{l_{1}} \rightarrow Y_{l_{1}}$. Also, for $u \in E, \alpha f_{l_{1}}(u)=\alpha(0, e(u))=(0, \xi(0)+e(u))=f_{l_{1}^{\prime}}(u)$ and $g_{l_{1}^{\prime}} \alpha\left(x, x_{0}\right)=g_{l_{1}}\left(x, \xi(x)+x_{0}\right)=x=g_{l_{1}}\left(x, x_{0}\right)$. Thus $\alpha f_{l_{1}}=f_{l_{1}^{\prime}}$ and $g_{l_{1}}=g_{l_{1}^{\prime}} \alpha$ so that $\alpha$ is an equivalence. However, since

$$
0 \longrightarrow E \stackrel{f}{\longrightarrow} Y \stackrel{g}{\longrightarrow} G \longrightarrow 0 \text { and } \quad 0 \longrightarrow E \stackrel{f_{l_{1}}}{\longrightarrow} Y_{l_{1}} \stackrel{g_{l_{1}}}{\longrightarrow} G \longrightarrow 0
$$


are equivalent, and

$$
0 \longrightarrow E \stackrel{f^{\prime}}{\longrightarrow} Y^{\prime} \stackrel{g^{\prime}}{\longrightarrow} G^{\prime} \longrightarrow 0 \quad \text { and } \quad 0 \longrightarrow E \stackrel{f_{l_{1}^{\prime}}}{\longrightarrow} Y_{l_{1}^{\prime}} \stackrel{g_{l_{1}^{\prime}}}{\longrightarrow} G \longrightarrow 0
$$

are equivalent, it follows that

$$
0 \rightarrow E \rightarrow Y \rightarrow G \rightarrow 0 \quad \text { and } \quad 0 \rightarrow E \rightarrow Y^{\prime} \rightarrow G \rightarrow 0
$$

are equivalent. Thus the map $\mathscr{E}(G, E) \rightarrow \operatorname{Ext}_{1}^{I}(G, E)$ is bijective.

If

$$
0 \longrightarrow E \stackrel{f}{\longrightarrow} Y \stackrel{g}{\longrightarrow} G \longrightarrow 0 \text { and } \quad 0 \longrightarrow E \stackrel{f^{\prime}}{\longrightarrow} Y^{\prime} \stackrel{g^{\prime}}{\longrightarrow} G \longrightarrow 0
$$

are two extensions of $E$ by $G$, one defines their Baer product as follows: Form $Y \oplus Y^{\prime}$, and let $A=\left\{\left(y, y^{\prime}\right):\left(y, y^{\prime}\right) \in Y \oplus Y^{\prime}, g(y)=g^{\prime}\left(y^{\prime}\right)\right\} . A$ is a closed subgroup of $Y \oplus Y^{\prime}$ since $g$ and $g^{\prime}$ are continuous homomorphisms. Let

$$
B=\left\{\left(-f(u), f^{\prime}(u)\right): u \in E\right\} .
$$

$B$ is a closed subgroup of $Y \oplus Y^{\prime}$ because $f$ and $f^{\prime}$ are proper homomorphisms. Since $g f=g^{\prime} f^{\prime}=0, B \subset A$. Hence $B$ is a closed subgroup of $A$. Let $Y^{\prime \prime}=A / B$ and $\pi: A \rightarrow Y^{\prime \prime}$ be the canonical epimorphism. If $u \in E$, then $(f(u), 0)$ and $\left(0, f^{\prime}(u)\right) \in A$. Define $f^{\prime \prime}(u)=\pi(f(u), 0)=\pi\left(0, f^{\prime}(u)\right)$. Then $f^{\prime \prime}: E \rightarrow Y^{\prime \prime}$ is a continuous homomorphism since $f$ and $\pi$ are. Let $\alpha: A \rightarrow G$ be defined by $\alpha\left(y, y^{\prime}\right)=g(y)=g^{\prime}\left(y^{\prime}\right)$. Since $\alpha$ is the composition of $g$ with the restriction to $A$ of a projection, it is a continuous homomorphism. Also, $\alpha\left(-f(u), f^{\prime}(u)\right)=g^{\prime}\left(f^{\prime}(u)\right)=0$. Therefore $\alpha$ induces a continuous homomorphism $g^{\prime \prime}: Y^{\prime \prime} \rightarrow G$. Since $G$ and $E$ are in $\mathscr{C}$, it follows from Theorem 2.6 that $Y$ and $Y^{\prime}$ are in $\mathscr{C}$. Hence $Y \oplus Y^{\prime}$ is in $\mathscr{C}$, by Proposition 2.6 and Corollary 1 of Theorem 2.5. Consequently $A$ and therefore $Y^{\prime \prime}$ is in $\mathscr{C}$ by Theorem 2.6 . It is a strictly formal fact that

$$
0 \longrightarrow E \stackrel{f^{\prime \prime}}{\longrightarrow} Y^{\prime \prime} \stackrel{g^{\prime \prime}}{\longrightarrow} G \longrightarrow 0
$$

is an exact sequence. Since $E, Y, G$ are in $\mathscr{C}$ it is proper. The equivalence class of this extension depends only on the equivalence classes of the given extensions. Thus $\mathscr{E}(G, E)$ is closed under Baer multiplication. It is also a strictly formal fact that the correspondence between $\mathscr{E}(G, E)$ and $\operatorname{Ext}_{1}^{I}(G, E)$ is a homomorphism. Hence $\mathscr{E}(G, E) \rightarrow \operatorname{Ext}_{1}^{I}(G, E)$ is an isomorphism. This completes the proof of Theorem 6.6( $\left.{ }^{8}\right)$.

Corollary. Let $0 \rightarrow E \rightarrow Y \rightarrow G \rightarrow 0$ be an extension with $G$ in $\mathscr{C}$ and $E$ elementary. If (1) The maximum compact subgroup of $G=(0)$, or

(2) $E$ is connected, or

(3) $G$ is torsion free and $E / E_{0}$ is finite, then $Y \cong G \oplus E$.

Let $0 \rightarrow H \rightarrow Y \rightarrow E \rightarrow 0$ be an extension with $H$ in $\mathscr{N}$ and $E$ elementary.

If (1) $H$ is connected, or

(2) the maximum compact subgroup of $E=(0)$, or

(3) $H$ is divisible and the toral dimension of $E=(0)$, then $Y \cong H \oplus E$.

$\left({ }^{8}\right)$ The proof of Theorem 6.6 is modeled after one in [6]. 
Proof. These corollaries follow directly from Theorem 6.6, the Corollary of Theorem 6.3 and Corollary 3 of Theorem 6.4.

\section{REFERENCES}

1. A. Weil, L'integration dans les groupes topologiques et ses applications, Hermann, Paris, 1953.

2. L. Pontrjagin, Topologische Gruppen, Teil 1, 2, Teubner, Leipzig, 1957.

3. I. Kaplansky, Infinite Abelian groups, The University of Michigan Press, Ann Arbor, 1954.

4. D. Montgomery and L. Zippin, Topological transformation groups, Interscience, New York, 1955.

5. J. Braconnier, Sur les groupes topologiques localement compacts, J. Math. Pures Appl. 27 (1948), 1-85.

6. H. Cartan and S. Eilenberg, Homological algebra, Princeton Univ. Press, Princeton, N. J., 1956.

7. J. Dixmier, Quelques propriétés des groupes abéliens localement compacts, Bull. Sci. Math. 81 (1957), 38-48.

8. H. Yamabe, On the conjecture of Iwasawa and Gleason, Ann. of Math. 58 (1953), 48-54.

9. A. Gleason, Groups without small subgroups, Ann. of Math. 56 (1952), 193-212.

\section{UNIVERSITY OF CALIFORNIA,}

Berkeley, California 